\title{
ANALYSIS OF THE SIMULATION OF SINGLE PHASE FLOW THROUGH A NATURALLY FRACTURED RESERVOIR
}

BY

TODD ARBOGAST

IMA Preprint Series \#295

February 1987 \section{UNIVERSITY OF MINNESOTA \\ 514 Vincent Hall \\ 206 Church Street S.E. \\ Minneapolis. Minnesota 55455}

INSTITUTE FOR MATHEMATICS AND ITS APPLICATIONS 


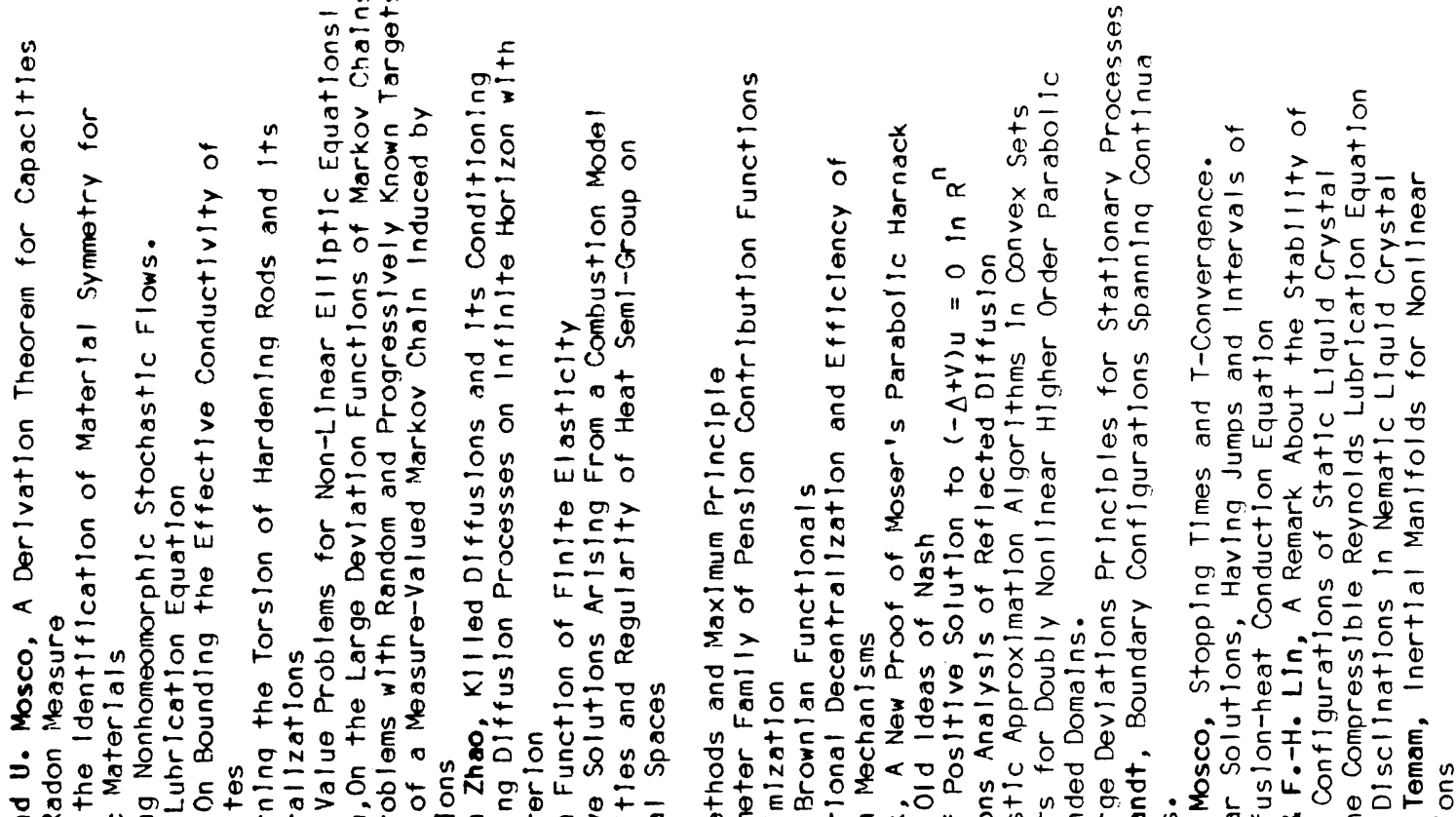

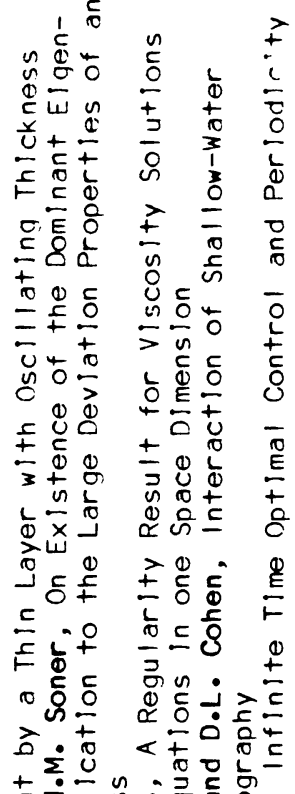

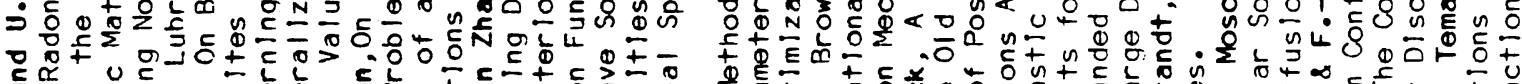

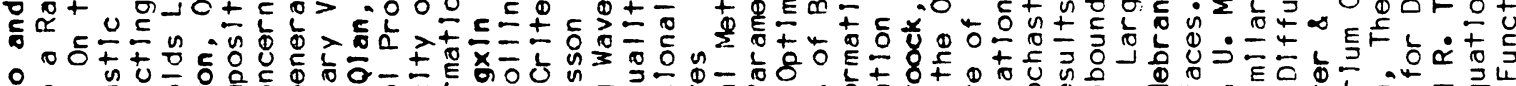

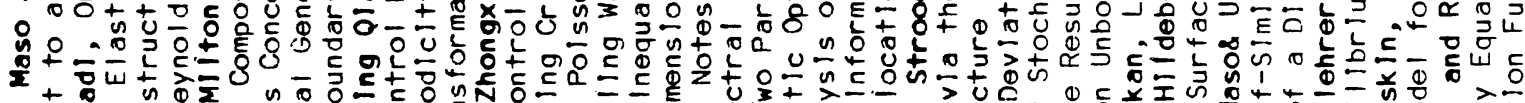

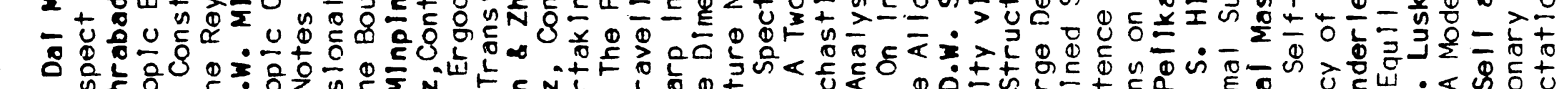

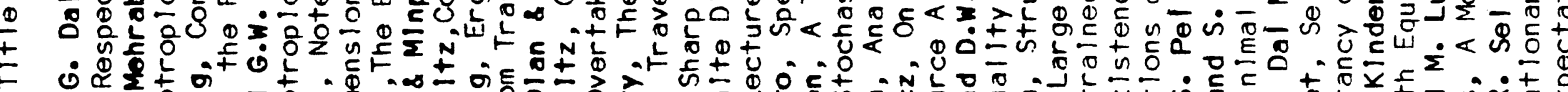

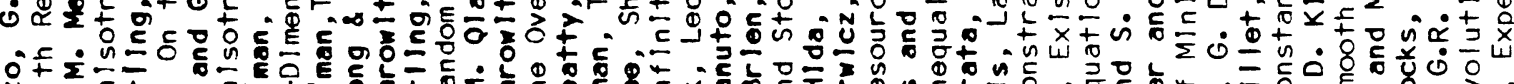

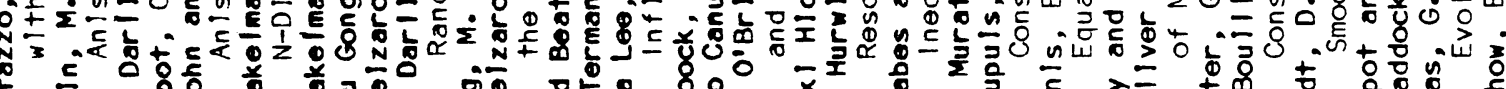

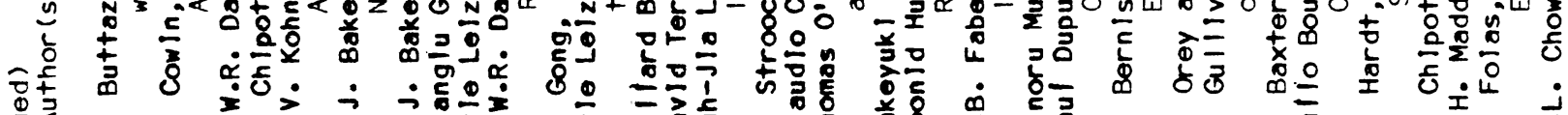

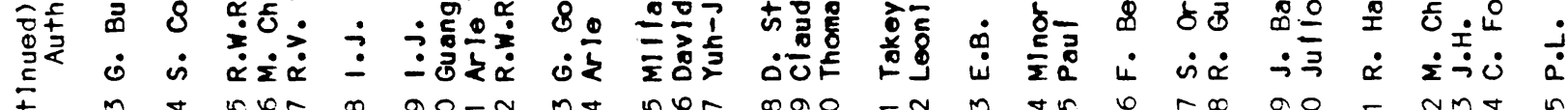

ర.

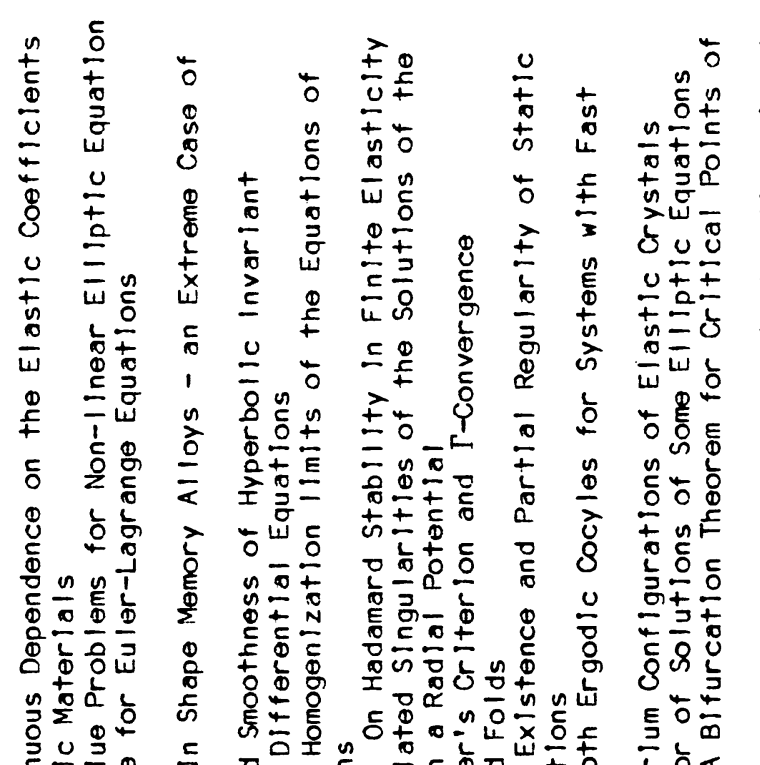
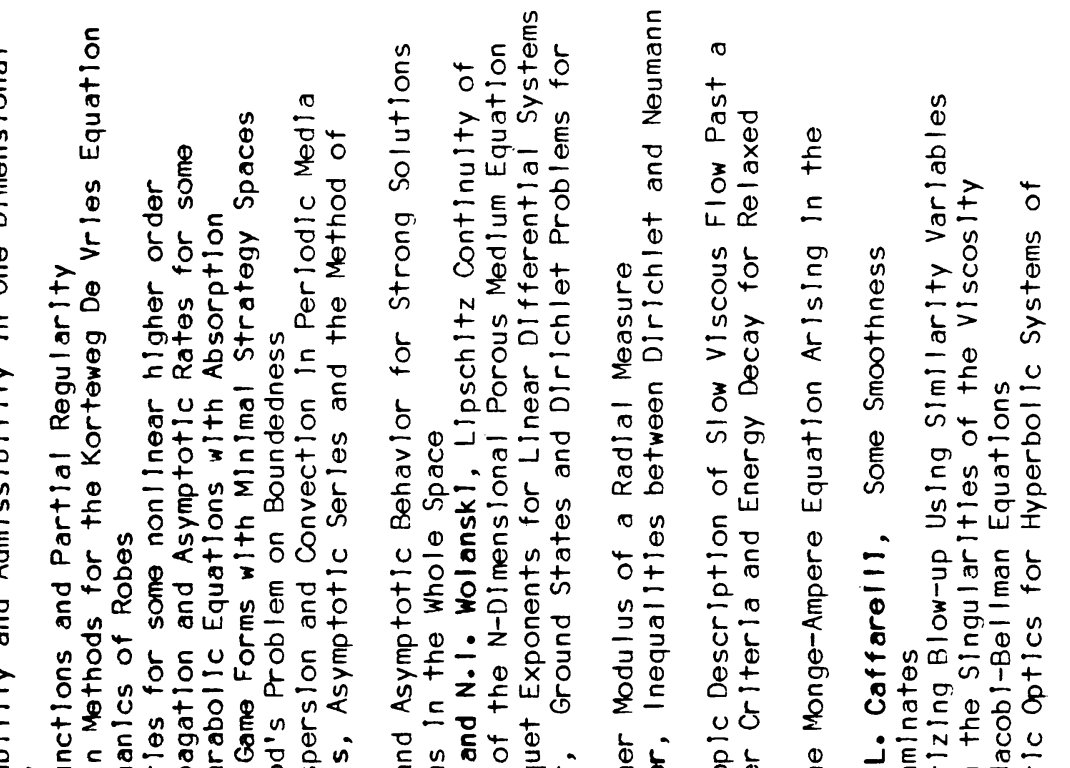

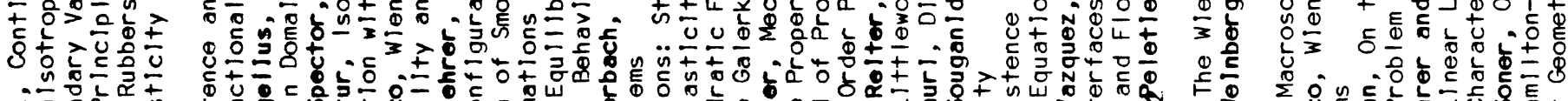

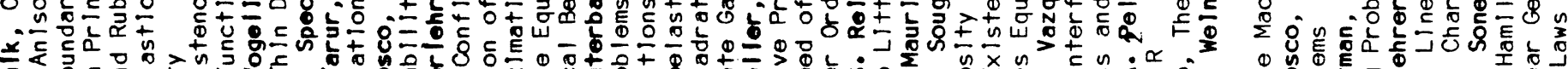

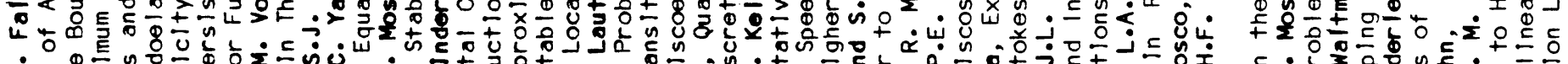

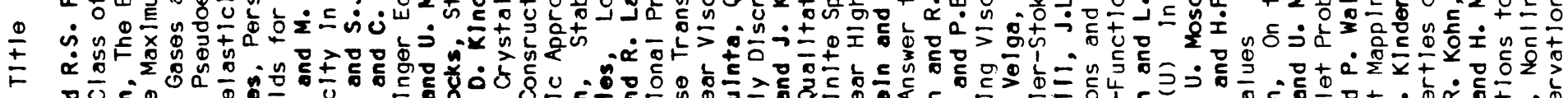

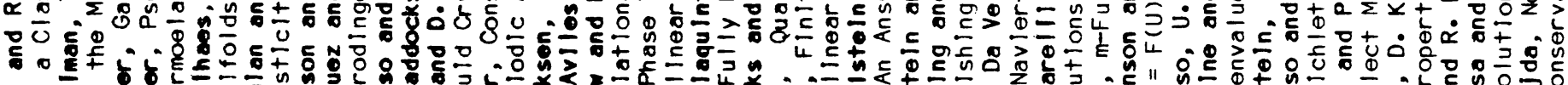

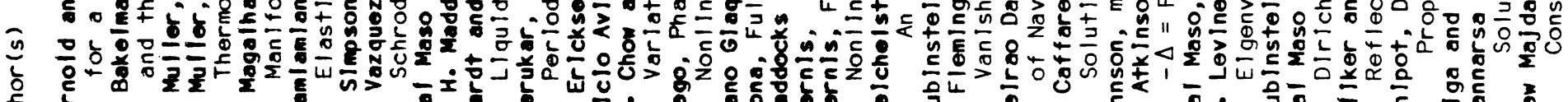




\title{
ANALYSIS OF THE SIMULATION OF SINGLE PHASE FLOW THROUGH A NATURALLY FRACTURED RESERVOIR
}

\author{
Todd Arbogast \\ Department of Mathematics \\ University of Chicago \\ Chicago, Illinois 60637
}

\begin{abstract}
The double porosity model for single phase flow through a naturally fractured reservoir is derived as a parabolic equation coupled to a series of parabolic equations. It is shown that the coupling term can be viewed as a positive-semidefinite perturbation of the time derivative, and hence it is verified that the model is well posed. A finite element method is presented to approximate the solution, and optimal order $\mathrm{L}^{2}$ error estimates are derived.
\end{abstract}

Key words: porous media, fractured reservoir, parabolic equation, finite element method.

AMS (MOS) subject classifications: $35 \mathrm{~K} 99,65 \mathrm{~N} 30,76505$.

\section{Introduction.}

The double porosity model of single phase flow through a naturally fractured reservoir was first described by Barenblatt, Zheltov, and Kochina [1], and Warren and Root [11], each of whom assumed a quasi-steady state flow in the matrix. Kazemi [5] and de Swaan 0. [10] considered the fully unsteady state model. We shall derive and analyze a somewhat more general form of this double porosity model.

A fractured reservoir may be idealized as a porous medium, or matrix, having some regular system of fractures. We consider a fractured reservoir $\Omega \subset \mathbb{R}^{d}, d=2$ or 3 , in which the fracture system decomposes the reservoir into a disjoint union of matrix blocks $\Omega_{i} \subset \Omega$; that is, $\bar{\Omega}=\mathrm{U}_{i} \bar{\Omega}_{i}$ and $\Omega_{i} \cap \Omega_{\mathrm{j}}=\varnothing, i \neq \mathrm{j}$. For a naturally fractured reservoir, we may assume that the diameter of each block is small compared to the dimensions of the reservoir itself.

Because the reservoir is so large, both the matrix and the fracture system have a macroscopically fine structure, each of which may be viewed as a distinct porous medium. The matrix blocks are ordinary porous media (with impermeable rock grains) for which the physics is well known (see [8] and [9]). 
The fracture system is a porous medium whose "grains" (the matrix blocks) are permeable. We consider the matrix blocks as sources (and sinks) within the fracture medium and apply the known physics to it. The macroscopic quantities porosity, (tensor) permeability, pressure, and density can be defined in the usual way with respect to the matrix as $\phi_{m}, k_{m}, P_{m}$, and $\rho_{m}$, respectively. The corresponding quantities for the fracture medium, $\phi, \mathbf{k}, p$, and $\rho$, can be defined similarly by considering the matrix blocks to be impermeable (cf. [1] and [11]).

Several physical assumptions need to be made:

1. The fluid is of constant compressibility; that is, it satisfies the equation of state

$$
\rho^{-1} d \rho=c d p
$$

where $c>0$ is a constant.

2. When gravity is present, the linearization approximation

$$
\rho(\underline{x}, t)^{2}=\left[\rho_{0}(\underline{x})+\left(\rho(\underline{x}, t)-\rho_{0}(\underline{x})\right)\right]^{2} \approx \rho_{0}(\underline{x})\left[2 \rho(\underline{x}, t)-\rho_{0}(\underline{x})\right]
$$

is valid, where $p_{0}(\underline{x})$ is some standard fluid density distribution.

3. Each matrix block interacts only with the fracture system; matrix blocks do not directly interact with each other, nor do they interact with any external source (or sink).

4. The fluid is assumed to be uniform at the surface of each matrix block.

5. The matrix porosity and permeability are constant over each block. For each i, $\phi_{m i}$ and $\mathbf{k}_{m i}$ shall denote $\phi_{m}$ and $\mathbf{k}_{m}$ restricted to $\Omega_{\mathrm{i}}$, respectively.

Assumptions 1 and 2 are customarily made in reservoir simulation. Assumptions 3 through 5 reflect the usual physical situation where the matrix blocks are relatively small and the matrix has a much higher storage capacity and a much lower flow capacity than the fracture system. Assumption 5 is not necessary for the analysis below. With the obvious modifications, all the results remain true if $\phi_{m}$ and $\mathbf{k}_{m}$ are allowed to be smooth functions of $\underline{x}$ (except, of course, the special case considered in Theorem 2). However, we shall retain this assumption to prevent the notation from becoming cumbersome.

In the fracture system, Darcy's law, conservation of mass, (1.1), and assumption 3 give us 


$$
\begin{aligned}
\phi(\underline{x}) \rho_{t}(\underline{x}, t)-\underline{\nabla} \cdot\left[\frac{k(x)}{\mu}\left[c^{-1} \underline{\nabla} p(\underline{x}, t)-p(\underline{x}, t)^{2} g \underline{\nabla} z(\underline{x})\right]=\right. & f(\underline{x}, t)+f_{m}(\underline{x}, t) \\
& \text { for }(\underline{x}, t) \in \Omega \times J,
\end{aligned}
$$

where the $t$ subscript denotes partial differentiation in time, $\mu$ is the viscosity of the fluid, $g$ is the gravitational constant, $z$ is the vertical coordinate, $f$ and $f_{m}$ are the external and matrix source terms, respectively, and $J=(0, T]$ is the time interval of interest. By assumption 2, we linearize (1.3) as

$$
\begin{gathered}
\phi(\underline{x}) \rho_{t}(\underline{x}, t)-\underline{\nabla} \cdot\left[\frac{k(x)}{\mu c} \nabla \rho(\underline{x}, t)-2 \rho_{0}(\underline{x}) g \frac{k(x)}{\mu} \nabla z(\underline{x})\left[\rho(\underline{x}, t)-1 / 2 \rho_{0}(\underline{x})\right]\right] \\
=f(\underline{x}, t)+f_{m}(\underline{x}, t) \quad \text { for }(\underline{x}, t) \in \Omega(x J .
\end{gathered}
$$

Since the boundary of a reservoir is generally ill-defined and of little consequence, we shall simply take the no flow Neumann condition there:

$$
\begin{aligned}
{\left[\frac{k(x)}{\mu C} \nabla \rho(\underline{x}, t)-2 \rho_{0}(\underline{x}) g \frac{k(x)}{\mu} \nabla \underline{\nabla} z(\underline{x})\left[\rho(\underline{x}, t)-1 / 2 \rho_{0}(\underline{x})\right]\right] \cdot \underline{\underline{p}(\underline{x})}=0 } \\
\text { for }(\underline{x}, t) \in \partial \Omega(x],
\end{aligned}
$$

where $\underline{v}$ is the outer normal to $\partial \Omega$. Finally, the initial fluid density

$$
\rho(\underline{x}, 0)=\rho^{0}(\underline{x}) \quad \text { for } \underline{x} \in \Omega
$$

must be given.

The $i$ th matrix block transmits through its surface an average flow of fluid

$$
f_{m i}(t)=-\frac{1}{\left|\Omega_{i}\right|} \int_{\partial \Omega_{i}} \frac{k_{m i}}{\mu c} \nabla \rho_{m} \cdot \underline{v} d s \quad \text { for } t \in J,
$$

where we use the notation $\left|\Omega_{i}\right|=\int_{\Omega_{i}} d x$ for the volume of $\Omega_{i}$. This flow should enter the fracture system near the block. In the continuous medium hypothesis, we locally average fine structure effects. This is implicitly done in defining the various macroscopic quantities above. To model the interchange between the two fine structures, we must explicitly describe the local averaging of the finer matrix structure with respect to the coarser fracture structure. Choose some partition of unity $\left\{X_{i}(x)\right\}$ over $\Omega$ such that each $X_{i}$ is approximately the characteristic function of $\Omega_{i}$ (i.e. each $X_{i}$ is supported near $\Omega_{i}, 0 \leq X_{i}, \int X_{i} d x=\left|\Omega_{i}\right|$, and 
$\left.\Sigma_{i} X_{i} \equiv 1\right)$. Now we can define the matrix source term as

$(1.5 b)$

$$
f_{m}(\underline{x}, t)=\sum_{i} f_{m i}(t) x_{i}(\underline{x}) \quad \text { for }(\underline{x}, t) \in \Omega \times J .
$$

The particular partition of unity used determines the exact manner in which the literal fracture flow around $\partial \Omega_{j}$ is marcoscopically spread out over the domain $\Omega$. The effect of this choice is to smooth out sharp variations in the solution $p$ that would otherwise arise near the boundary of each $\Omega_{i}$ (see Theorem 4 below). In practice, one never looks at the solution in such detail, so this choice is not actually very important.

Analogous to (1.3), we have in the ith matrix block

$$
\phi_{m i} \rho_{m, t}(\underline{x}, t)-\underline{\nabla} \cdot\left[\frac{k_{m}}{\mu c} \underline{\nabla} \rho_{m}(\underline{x}, t)\right]=0 \quad \text { for }(\underline{x}, t) \in \Omega_{j} \times J .
$$

Here we have omitted the gravitational term, since the effects of assumption 4 completely dominate it. Continuity of pressure requires that the block and fracture densities be equal at the surface of the block, while initial equilibrium requires the same equality at time zero. By assumption 4 , at each time $t$, we can simply take a constant, $\hat{p}_{j}(t)$, to represent the density of the fluid in the fractures surrounding the ith block. That is, we can write

$$
\rho_{m}=\hat{\rho}_{i} \quad \text { on } \partial \Omega_{i} \times J
$$

(where the trace is taken from inside $\Omega_{j}$ ) and

$$
\rho_{m}(\underline{x}, 0)=\hat{\rho}_{i}^{0} \quad \text { for } \underline{x} \in \Omega_{i} \text {. }
$$

Finally, we should def ine these constants according to

$$
\hat{u}_{i}=\left|\Omega_{i}\right|^{-1} \int_{\Omega} u X_{i} d x \quad \text { for } u \in L^{1}(\Omega) \text {. }
$$

This definition gives us consistency between the local ( $i$ th block) behavior of the fracture flow in (1.5b) and in $(1.6 \mathrm{~b}, \mathrm{c})$.

Equations (1.4) through (1.7) constitute our model. In the next section, we shall specify the mathematical assumptions as well as define some general notation. In section 3 we analyze the matrix source term. In particular, we show that it is positive-semidefinite in some sense. In section 4 we show that 
the model is mathematically well posed. A finite element method of approximating the solution $\rho$ (and $\rho_{m}$ ) is presented and analyzed in sections 5 and 6 .

\section{General assumptions and notations.}

In the rest of the paper we shall use the following assumptions and notations concerning the coefficients. Assume that $f, \rho_{0}, \rho^{0}$, and the $X_{i}$ are smoothly distributed over the reservoir and sufficiently regular. The (unphysical) assumption on $f$ will ensure that the solution $\rho$ has the necessary smoothness and $L^{2}$-norm properties for the analysis below. Let $\phi(\underline{x})$ and $\phi_{m}$ be bounded above and below by positive constants over $\Omega$ and $\Omega_{m}$ (where $\Omega_{m}=u_{i} \Omega_{j}$ ), respectively, and suppose that $\phi$ is smooth. Let $\mathbf{x}(\underline{x})=\mathbf{k}(\underline{x}) / \mu \mathrm{c}$ and $\mathbf{x}_{m}=\mathbf{k}_{m} / \mu \mathrm{c}$ be symmetric and uniformly positive-definite matrices over $\Omega$ and $\Omega_{m}$, respectively, such that $x$ is smooth and sufficiently regular. Finally, let $\underline{\gamma}(x)=$ $2 p_{0}(\underline{x}) g k(\underline{x}) \underline{\nabla} z(\underline{x}) / \mu$.

We will assume that $\Omega$ is a bounded domain with smooth boundary. Obviously we may not make the same assumption on $\Omega_{j}$; instead, we will assume that $\Omega_{\mathrm{i}}$ is a convex domain, for each i. Hence, $\partial \Omega_{\mathrm{j}}$ is plecewise smooth.

$W^{P}, r, 0 \leq p$ and $1 \leq r \leq \infty$, will denote the usual Sobolev space of $p$ times differentiable functions in $L^{r}$. Let $H^{P}=W^{P}, 2$. $H^{-D}$ will denote the dual of $H^{P}$. $H^{q}(J ; H P)$, q a nonnegative integer, will denote the Banach space of $H^{P}$-valued functions (of $\underline{x}$ ) that are in $\mathrm{H}^{\mathrm{Q}}$ (as functions of $\mathrm{t}$ ). The norm of $\mathrm{U}(\underline{x}, \mathrm{t}) \in \mathrm{H}^{\mathrm{q}}\left(\mathrm{J} ; \mathrm{H}^{\mathrm{P}}\right)$ is given by

$$
\|u\|_{H} q(J ; H P)=\left\|\sum_{j=0}^{q}\right\| \partial j_{u} / \partial t\left\|_{H}\right\|_{H} \|_{L^{2}(J)} \text {. }
$$

Similarly we have the space $L^{\infty}(\mathrm{J}: H P)$. We will also need the spaces $H_{0}^{1}\left(\Omega_{j}\right)=$ $\left\{v \in H^{\prime}\left(\Omega_{i}\right): v=0\right.$ on $\left.\partial \Omega_{j}\right\}$.

We will denote by $(\cdot, \cdot)$ the inner product on $L^{2}(\Omega)$ or $\left(L^{2}(\Omega)\right)^{d}$, whichever is appropriate. We will similarly denote by $(\cdot, \cdot)_{i}$ the inner product on $L^{2}\left(\Omega_{\mathrm{i}}\right)$ or $\left(L^{2}\left(\Omega_{j}\right)\right)^{d}$, and by $(\cdot, \cdot)_{m}$ the inner product on $L^{2}\left(\Omega_{m}\right)$ or $\left(L^{2}\left(\Omega_{m}\right)\right)^{d}$. Define the symmetric bilinear forms

$$
\begin{array}{ll}
B\left(u_{1}, u_{2}\right)=\left(x \underline{\nabla} u_{1}, \underline{\nabla} u_{2}\right) & \text { for any } u_{1}, u_{2} \in H^{\prime}(\Omega), \\
B_{1}\left(v_{1}, v_{2}\right)=\left(\mathbf{x}_{m} \underline{\nabla} v_{1}, \underline{\nabla} v_{2}\right)_{1} & \text { for any } v_{1}, v_{2} \in H^{\prime}\left(\Omega_{1}\right), \text { for each i, }
\end{array}
$$


and

$$
B_{m}\left(v_{1}, v_{2}\right)=\left(x_{m} \underline{\nabla} v_{1}, \underline{\nabla} v_{2}\right)_{m} \quad \text { for any } v_{1}, v_{2} \in H^{1}\left(\Omega_{m}\right)
$$

Finally, $C$ and $\epsilon$ will denote generic positive constants.

Let us write the differential equations of the model in weak form with the above notation. First, note that by applying the divergence theorem to (1.6a), we can rewrite (1.5a) as

$$
f_{m i}(t)=-\phi_{m i} \bar{\rho}_{m, t, i}
$$

with the notation

$$
\bar{v}_{i}=\left|\Omega_{i}\right|^{-1} \int_{\Omega_{i}} v d x
$$

for the average of $v \in L^{\prime}\left(\Omega_{i}\right)$ over $\Omega_{j}$. Now we can write $(1.4 a, b)$ and $(1.5)$ as

$$
\begin{gathered}
\left(\phi \rho_{t}, \varphi\right)+\sum_{i} \phi_{m i} \bar{\rho}_{m, t, i} \hat{\varphi}_{i}\left|\Omega_{i}\right|+B(\rho, \varphi)-(\underline{\gamma} \rho, \underline{\nabla} \varphi) \\
=(f, \varphi)-1 / 2\left(\underline{\gamma} \rho_{0}, \underline{\nabla} \varphi\right) \quad \text { for } \varphi \in H^{1}(\Omega),
\end{gathered}
$$

and, for each i, (1.6a) as

$$
\phi_{m i}\left(\rho_{m, t}, \psi\right)_{i}+B_{i}\left(\rho_{m}, \psi\right)=0 \quad \text { for } \psi \in H^{1}{ }_{0}\left(\Omega_{i}\right)
$$

of course, the boundary condition (1.6b) must be imposed on $\mathrm{Pm}_{\mathrm{m}}$.

\section{Description of the matrix source term.}

For a given $\rho$ in $H^{1}\left(J ; L^{2}(\Omega)\right.$ ), the solution $\rho_{m}$ of $(1.6)$ (or $(2.3),(1.6 b, c)$ ) exists and is unique as a function in $H^{\prime}\left(J ; L^{2}\left(\Omega_{m}\right)\right) \cap L^{2}\left(J ; H^{2}\left(\Omega_{m}\right)\right)$, since $\Omega_{i}$ is convex for each $i$. As a consequence. we can define the matrix source term operator $F_{m}: L^{2}\left(J: L^{2}(\Omega)\right) \rightarrow L^{2}\left(J ; L^{2}(\Omega)\right)$ as

$$
F_{m}\left(u_{*}\right)=\sum_{i} \phi_{m i} \bar{v}_{t, i} x_{i}
$$

where, on $\Omega_{i}$, v satisfies 


$$
\phi_{m i}\left(v_{t}, \psi\right)_{i}+B_{i}(v, \psi)=0 \quad \text { for } \psi \in H^{1}{ }_{0}\left(\Omega_{i}\right)
$$

$$
v=\int_{0}^{t} \hat{u}_{*, i}(\tau) d \tau \quad \text { on } \partial \Omega_{j} \times J
$$

$$
V(\underline{x}, 0)=0 \quad \text { for } \underline{x} \in \Omega_{j} .
$$

Note that $u_{*}=\rho_{t}$ implies that $v_{t}=\rho_{m}, t$; hence, $F_{m}\left(\rho_{t}\right)=-f_{m}$ represents flow from the fracture system to the matrix. We should expect that $\rho_{t}$ and $F_{m}\left(\rho_{t}\right)$ behave similarly, at least in some average sense. This is expressed as

Theorem 1:

$F_{m}$ is a bounded linear positive-semidefinite operator from $L^{2}\left(\mathcal{J}_{;} L^{2}(\Omega)\right)$ to $L^{2}\left(\tilde{J}: L^{2}(\Omega)\right)$ for any subinterval $\tilde{J}=(0, \tau] \subset J$. That is,

$$
\left\|F_{m}\left(U_{*}\right)\right\|_{L^{2}\left(\tilde{J} ; L^{2}(\Omega)\right)} \leq C\left\|u_{*}\right\|_{L^{2}\left(\tilde{J} ; L^{2}(\Omega)\right)}
$$

and

$$
0 \leq \int_{0}^{\tau}\left(F_{m}\left(u_{*}\right), u_{*}\right) d t
$$

for any $u_{*} \in L^{2}\left(\tilde{J} ; L^{2}(\Omega)\right)$. Moreover,

$$
-C\|u(\cdot, 0)\|^{2} L^{2}(\Omega) \leq \int_{0}^{\tau}\left(F_{m}\left(u_{t}\right), u\right) d t
$$

for any $u \in H^{1}\left(\tilde{J} ; L^{2}(\Omega)\right)$.

Proof:

Take $\psi=v_{t}-\hat{U}_{*}, i$ in (3.2a) and integrate over $\tilde{J}$ to see

$$
\begin{aligned}
& \phi_{m i}\left\|v_{t}\right\|^{2} L^{2}\left(\tau_{;} L^{2}\left(\Omega_{j}\right)\right)+1 /{ }_{2} B_{i}(v, v)(\tau) \\
& =\phi_{m i} \int_{0}^{\tau} \bar{v}_{t, i} \hat{u}_{*}, \mathrm{~d} d \mathrm{t}\left|\Omega_{\mathrm{i}}\right| \\
& \leq 1 / 2 \phi_{m i}\left\|V_{t}\right\|^{2} L^{2}\left(\tilde{J}_{i} L^{2}\left(\Omega_{i}\right)\right)+1 / 2 \phi_{m i}\left\|u_{*} X_{i}{ }^{1 / 2}\right\|^{2} L^{2}\left(\tilde{J}_{i} L^{2}(\Omega)\right) \text {. }
\end{aligned}
$$

Canceling terms above, we obtain boundedness: 
(3.7)

$$
\begin{aligned}
& \left\|F_{m}\left(u_{*}\right)\right\|_{L^{2}\left(\tau_{j} L^{2}(\Omega)\right)} \\
& \quad=\|\| \sum_{i} \phi_{m i} \bar{v}_{t, i} x_{i}\left\|_{L^{2}(\Omega)}\right\|_{L^{2}(\Im)}
\end{aligned}
$$

$$
\begin{aligned}
& \leq \sum_{i} \phi_{m i}\left\|v_{t}\right\|_{L^{2}\left(\widetilde{J} ; L^{2}\left(\Omega_{i}\right)\right)} \\
& \leq C\left\|U_{*}\right\|_{L^{2}\left(\widetilde{J}_{;} L^{2}(\Omega)\right)} .
\end{aligned}
$$

Using only the equality in (3.6), we get positive-semidef initeness:

$$
\begin{aligned}
& \int_{0}^{\tau}\left(F_{m}\left(u_{*}\right), u_{*}\right) d t \\
& \quad=\sum_{i} \phi_{m i} \int_{0}^{\tau} \bar{v}_{t, i} \hat{u}_{*, i} d t\left|\Omega_{i}\right| \\
& \quad \geq 0 .
\end{aligned}
$$

To obtain (3.5), take $\psi=v-\int_{0}{ }_{0} \hat{u}_{t, i} d \tau=\left(v+\hat{u}_{i}(0)\right)-\hat{u}_{i}$ in (3.2a) and integrate over $\widetilde{J}$ to see that

$$
\begin{aligned}
& 1 / 2 \phi_{m i}\left\|v+\hat{u}_{i}(0)\right\|_{L^{2}\left(\Omega_{i}\right)}(\tau)-1 / 2 \phi_{m i}\left(\hat{u}_{i}(0)\right)^{2}\left|\Omega_{i}\right|+\int_{0}{ }^{2} B_{i}(v, v) d t \\
& \quad=\phi_{m i} \int_{0}^{\tau} \bar{v}_{t, i} \hat{u}_{i} d t\left|\Omega_{i}\right| .
\end{aligned}
$$

and then estimate

$$
\begin{aligned}
& \int_{0}^{\tau}\left(F_{m}\left(u_{t}\right), u\right) d t \\
& \quad=\sum_{i} \phi_{m i} \int_{0} \tau \bar{v}_{t, i} \hat{u}_{i} d t\left|\Omega_{i}\right| \\
& \quad \geq-C\|u(\cdot, 0)\|^{2}{ }^{2}(\Omega) .
\end{aligned}
$$

A more complete understanding of the matrix source term operator may be obtained by expressing it explicitly in terms of the auxiliary function $\theta$ which, on $\Omega_{\mathrm{j}}$, is the solution to

$$
\phi_{m i} \theta_{t}-\underline{\nabla} \cdot\left(x_{m} \underline{\nabla} \theta\right)=0 \quad \text { on } \Omega_{i} \times J,
$$

$$
\theta=1 \quad \text { on } \partial \Omega_{\mathrm{i}} \times \mathrm{J} \text {, }
$$


(3.11c) $\quad \theta(\underline{x}, 0)=0 \quad$ for $\underline{x} \in \Omega_{j}$.

The solution of (1.6) is the convolution in time

$$
\rho_{m}(\underline{x}, t)=\int_{0}^{t} \theta(\underline{x}, t-\tau) \hat{\rho}_{t, i}(\tau) d \tau+\hat{\rho}_{i} \quad \text { for }(\underline{x}, t) \in \Omega_{i} x J,
$$

and so

$$
F_{m}\left(p_{t}\right)(\underline{x}, t)=\sum_{i} \phi_{m i} \int_{0}{ }^{t} \bar{\theta}_{t, i}(t-\tau) \hat{\rho}_{t, i}(\tau) d \tau X_{i}(\underline{x})
$$

This, in fact, allows us to decouple the system (1.4) through (1.7). Since $\bar{\theta}_{t, i}$ is positive, decreasing, and convex, the Fourier transform of its even extension to $\mathbb{R}$ is positive. An application of Fourier analysis to (3.13) gives an alternate proof of (3.4). This approach enables us to see that, in fact. $\left\{\int_{0}^{\top}\left(F_{m}(\cdot) \cdot \cdot\right) d t\right\}^{1 / 2}$ is a seminorm on $L^{2}\left(J ; L^{2}(\Omega)\right)$ equivalent to the sum on $i$ of the $\mathrm{H}^{-1 / 4}$-norms in time of the local space averages, at least in the following special (Dut very reasonable) case:

\section{Theorem 2:}

Suppose that each $\Omega_{\mathrm{i}}$ is a rectangular parallelepiped whose edges are parallel to the coordinate axes, and that each $\boldsymbol{x}_{\mathrm{mi}}$ is diagonal. Then there exist positive constants $C_{1}$ and $C_{2}$ (independent of $\rho_{t}$ ) such that

$$
\begin{aligned}
& C_{1} \sum_{i}\left\|\hat{\rho}_{t, i}\right\|^{2} H^{-1 / 4}(\mathrm{~J})\left|\Omega_{i}\right| \\
& \leq \int_{0}^{\top}\left(F_{m}\left(\rho_{t}\right), \rho_{t}\right) d t \\
& \leq C_{2} \sum_{i}\left\|\hat{\rho}_{t, i}\right\|^{2} H^{-1 / 4(J)}\left|\Omega_{i}\right| .
\end{aligned}
$$

Proof:

For each $\mathrm{i}$, an appropriate scaling allows us to assume that $\Omega_{i}=(0, \pi)^{d}+\underline{c}_{i}$. Write

$$
\frac{1}{\phi_{m i}} x_{m i}=\left[\begin{array}{lll}
k_{1} & & 0 \\
& \ddots & \\
0 & & k_{d}
\end{array}\right] .
$$

Separation of variables applied to (3.11) gives us 


$$
\bar{\theta}_{t, i}=\left[\frac{8}{\pi^{2}}\right]_{\lambda_{1}, \ldots, \lambda_{d}}^{d} \frac{\sum_{j} \lambda_{j}{ }^{2} k_{j}}{\prod_{j} \lambda_{j}^{2}} \exp \left(-\sum_{j} \lambda_{j}{ }^{2} k_{j} t\right),
$$

where $\Sigma_{0}$ denotes the sum over positive odd indices.

Let $\omega(t)=\bar{\theta}_{t, i}(|t|), t \in \mathbb{R}$, and let $\mathbf{F}$ denote the Fourier transform operator. It is trivial to calculate

$$
\mathbf{F} \omega(s)=2\left[\frac{8}{\pi^{2}}\right]_{\lambda_{1}, \ldots, \lambda_{d}}^{d} \frac{\left(\sum_{j} \lambda_{j}{ }^{2} k_{j}\right)^{2}}{\left(\prod_{j} \lambda_{j}{ }^{2}\right)\left[\left(\sum_{j} \lambda_{j}{ }^{2} k_{j}\right)^{2}+s^{2}\right]} .
$$

We will now show that there exist positive constants $\tilde{\mathrm{C}}_{1}$ and $\tilde{\mathrm{C}}_{2}$ such that

$$
\tilde{C}_{1}\left(1+s^{2}\right)^{-1 / 4} \leq F \omega(s) \leq \tilde{C}_{2}\left(1+s^{2}\right)^{-1 / 4}
$$

Since $\mathbf{F} \omega$ is continuous (Riemann-Lebesgue lemma) and even, it suffices to show the result for $s$ large. In that case,

$$
\begin{aligned}
\mathbf{f} \omega(s) & \geq c \sum_{\lambda_{1}} \frac{\left(\lambda_{1}{ }^{2}+d-1\right)^{2}}{\lambda_{1}{ }^{2}\left[\left(\lambda_{1}{ }^{2}+d-1\right)^{2}+s^{2}\right]} \\
& \geq \quad c \sum_{0} \frac{\lambda_{1}{ }^{2}}{\lambda_{1}{ }^{4}+s^{2}} \\
& 1 / 2_{2} s \leq \lambda_{1}{ }^{2} \leq 3 /{ }_{2} s \\
& \geq C s^{-1 / 2},
\end{aligned}
$$

as $\lambda_{1}=O\left(s^{1 / 2}\right)$ and there are $O\left(s^{1 / 2}\right)$ terms in the last sum, and

$$
\begin{aligned}
& \left(\lambda_{1}{ }^{2}+\sum_{j=2}^{d} \lambda_{j}^{2}\right)^{2} \\
& \boldsymbol{F} \omega(s) \leq \sum_{\substack{c \\
\lambda_{2}, \ldots, \lambda_{d}}} \sum_{\substack{\lambda_{1} \geq \max \left(\lambda_{2}, \ldots, \lambda_{d}\right) \\
\lambda_{1}}} \\
& \lambda_{1}{ }^{2}\left(\prod_{j=2}^{d} \lambda_{j}^{2}\right)\left[\left(\lambda_{1}{ }^{2}+\sum_{j=2}^{d} \lambda_{j}{ }^{2}\right)^{2}+s^{2}\right] \\
& \leq C\left[\sum_{\lambda_{2}, \ldots, \lambda_{d}} \frac{1}{\prod_{j=2}^{d} \lambda_{j}{ }^{2}}\right]\left[\sum_{\lambda_{1}} \frac{\lambda_{1}{ }^{2}}{\lambda_{1}{ }^{4}+s^{2}}\right]
\end{aligned}
$$




$$
\begin{aligned}
& \leq C \sum_{m=-\infty}^{\infty} \sum_{\substack{\lambda_{1} \\
2_{S \leq \lambda_{1}} \leq 2^{m+1} s}} \frac{\lambda_{1}{ }^{2}}{\lambda_{1}{ }^{4}+s^{2}} \\
& \leq C\left[\sum_{m=-\infty}^{\infty} \frac{2^{m}}{\left(2^{2 m+1)}\right.} 2^{m / 2}\right] s^{-1 / 2} \\
& \leq C s^{-1 / 2} .
\end{aligned}
$$

This establishes (3.17).

Our estimate (3.14) now follows directly from (3.13), some Fourier analysis, and (3.17):

$$
\begin{aligned}
& \int_{0}^{T}\left(F_{m}\left(\rho_{t}\right), \rho_{t}\right) d t \\
&= \sum_{i} \int_{0}^{T} \int_{0}^{t} \omega(t-\tau) \hat{\rho}_{t, i}(\tau) \hat{\rho}_{t, i}(t) d \tau d t\left|\Omega_{i}\right| \\
&= \sum_{i} \iint\left[\omega(t-\tau) X_{(0, \infty)}(t-\tau)\right]\left[\hat{\rho}_{t, i}(\tau) X_{J}(\tau)\right] \times \\
& \quad \times\left[\hat{\rho}_{t, i}(t) X_{J}(t)\right] d \tau d t\left|\Omega_{i}\right| \\
&= \sum_{i} \int F\left(\omega X_{(0, \infty)}\right)(s)\left|\mathbf{F}\left(\hat{\rho}_{t, i} X_{J}\right)(s)\right|^{2} d s\left|\Omega_{i}\right| \\
&=1 / 2 \sum \int \mathbf{F} \omega(s)\left|\mathbf{F}\left(\hat{\rho}_{t, i} X_{J}\right)(s)\right|^{2} d s\left|\Omega_{i}\right|
\end{aligned}
$$

which is then equivalent to

$$
\sum_{i} \int\left(1+s^{2}\right)^{-1 / 4}\left|\mathbf{F}\left(\hat{\rho}_{t, i} X_{J}\right)(s)\right|^{2} d s\left|\Omega_{i}\right|
$$

Since the integral above is equivalent to the square of the $\mathrm{H}^{-1 / 4}$-norm, the proof is complete.

As a final remark in this section, we will compare the so called Warren and Root model ([1] and [11]) with the present model. The quasi-steady state assumption, that $\rho_{m, t}=-\alpha\left(\rho_{m}-\rho\right)$, simply says that

$$
\rho_{m}(\underline{x}, t)=\int_{0}^{t}\left(1-e^{-\alpha(t-\tau)}\right) \rho_{t}(\underline{x}, \tau) d \tau+\rho^{O}(\underline{x}) \quad \text { for }(\underline{x}, t) \in \Omega \times J \text {. }
$$

A glance at (3.12) shows that this assumption essentially amounts to a one parameter approximation of $\theta$ (or $\bar{\theta}_{j}$ ) by a single time exponentiai. 
4. Demonstration that the model is well posed.

We will now show the existence, uniqueness, and continuous dependence on the data $\left(f, \rho_{0}, \rho^{0}\right)$ of the solution $\left(\rho, \rho_{m}\right)$. This will be accomplished by applying the method of continuity to the problem (1.4) decoupled from (1.6) via (3.13). The linearization assumption (1.2) creates some difficulties when gravity is present. The equations are not strictly linear, and a given solution $u$ can arise from many different sets of data, as the reference density function $\rho_{0}$ is allowed to be arbitrary. To resolve these difficulties, we shall analyze the equation over a smaller data space. We must be careful to determine the dependence on $\underline{\gamma}$, since $\underline{\gamma}$ is proportional to $\rho_{0}$.

For each $\lambda \in[0,1]$, consider the problem

$$
\begin{aligned}
\phi(\underline{x}) u_{t}(\underline{x}, t)+\lambda F_{m}\left(u_{t}\right)(\underline{x}, t)-\underline{\nabla} \cdot[x(\underline{x}) \underline{\nabla} u(\underline{x}, t)-\underline{\gamma}(\underline{x}) u(\underline{x}, t)]=F(\underline{x}, t) \\
\\
\text { for }(\underline{x}, t) \in \Omega x J,
\end{aligned}
$$

$$
[x(\underline{x}) \underline{\nabla} u(\underline{x}, t)-\underline{\gamma}(\underline{x}) u(\underline{x}, t)] \cdot \underline{v}(\underline{x})=u_{0}(\underline{x}) \quad \text { for }(\underline{x}, t) \in \partial \Omega x J,
$$

$$
u(\underline{x}, 0)=u^{0}(\underline{x}) \quad \text { for } \underline{x} \in \Omega \text {. }
$$

When $\lambda=0$, we have a standard well posed problem (see [6], Theorem 5.3, p. 32); when $\lambda=1$, we have our problem (1.4), where $F(\underline{x}, t)=f(\underline{x}, t)+1 / 2 \underline{\nabla} \cdot\left[\rho_{0}(\underline{x}) \underline{\gamma}(\underline{x})\right]$. $u_{0}(\underline{x})=-1 / 2 \rho_{0}(\underline{x}) \underline{\gamma}(\underline{x}) \cdot \underline{v}(\underline{x})$, and $u^{0}(\underline{x})=\rho^{0}(\underline{x})$.

Let

$$
\begin{aligned}
v=\left\{u \in H^{\prime}\left(J: L^{2}(\Omega)\right) \cap L^{2}\left(J: H^{2}(\Omega)\right):\right. \\
\quad(x \underline{\nabla} u-\underline{\gamma} u) \cdot \underline{v} \text { on } \partial \Omega \times J \text { is independent of } t\}
\end{aligned}
$$

and

$$
\begin{aligned}
\boldsymbol{w}=\left\{\left(F, u_{0}, u^{0}\right) \in L^{2}\left(J ; L^{2}(\Omega)\right) \times H^{1 / 2}(\partial \Omega) \times H^{1}(\Omega):\right. & \\
& \left.\left(\boldsymbol{x} \underline{\nabla} u^{0}-\underline{\gamma} u^{0}\right) \cdot \underline{v}=u_{0} \text { on } \partial \Omega\right\} .
\end{aligned}
$$

We can define for each $\lambda$ the linear operator

$$
\Phi_{\lambda}: v \rightarrow w
$$

representing (4.1) as

$$
\Phi_{\lambda}(u)=\left(\phi u_{t}+\lambda F_{m}\left(u_{t}\right)-\underline{\nabla} \cdot(x \underline{\nabla} u-\underline{\gamma} u),(x \underline{\nabla} u-\underline{\gamma} u) \cdot \underline{v}, u(\cdot, 0)\right),
$$


where the two trace operators are known to be bounded ([6], Theorem 2.1, p. 9).

\section{Lemma:}

The $\Phi_{\lambda}$ are bounded above and below with bounds that are independent of $\lambda \in[0,1]$. These bounds depend on $\underline{\gamma}$ only through its $H^{1}(\Omega)$-norm.

The following technical lemma will enable us to verify the $\underline{\gamma}$ dependence. It will be used with $\psi=\underline{\gamma}$ several times in the proof of the lemma.

Technical lemma:

If the dimension $d \leq 3$, then, for any $\epsilon>0$,

$$
\left(\Psi \varphi_{1}, \varphi_{2}\right) \leq C\|\Psi\|^{4} H^{1}(\Omega)\left\|\varphi_{1}\right\|^{2} L^{2}(\Omega)+\epsilon\left\{\left\|\varphi_{1}\right\|^{2} H^{1}(\Omega)+\left\|\varphi_{2}\right\|^{2} L^{2}(\Omega)\right\}
$$

and

$$
\|\underline{\nabla}(\psi \varphi)\|_{L^{2}(\Omega)} \leq C\|\psi\|^{2} H^{1}(\Omega)\|\varphi\|_{H^{1}(\Omega)}+\epsilon\|\varphi\|_{H^{2}(\Omega)} .
$$

Proof:

By Hölder's inequality and the Sobolev imbedding theorem, we have:

$$
\begin{aligned}
& \left(\Psi \varphi_{1}, \varphi_{2}\right) \leq\|\Psi\|_{L^{6}(\Omega)}\left\|\varphi_{1}\right\|_{L^{3}(\Omega)}\left\|\varphi_{2}\right\|_{L^{2}(\Omega)} \\
& \leq\|\Psi\|_{L^{6}(\Omega)}\left\|\varphi_{1}\right\|^{1 / 2} L^{2}(\Omega)\left\|\varphi_{1}\right\|^{1 / 2} L^{6}(\Omega)\left\|\varphi_{2}\right\|_{L^{2}(\Omega)} \\
& \leq C\|\psi\|_{H^{\prime}(\Omega)}\left\|\varphi_{1}\right\|^{1 / 2} L^{2}(\Omega)\left\|\varphi_{1}\right\|^{1 / 2} H^{\prime}(\Omega)\left\|\varphi_{2}\right\|_{L^{2}(\Omega)} \\
& \leq C\|\Psi\|^{4} H^{\prime}(\Omega)\left\|\varphi_{1}\right\|^{2} L^{2}(\Omega)+\epsilon\left\{\left\|\varphi_{1}\right\|^{2} H^{\prime}(\Omega)+\left\|\varphi_{2}\right\|^{2} L^{2}(\Omega)\right\} \text {. }
\end{aligned}
$$

Similarly,

$$
\begin{aligned}
\|\underline{\nabla}(\Psi \varphi)\|_{L^{2}(\Omega)} \leq & \|(\underline{\nabla} \Psi) \varphi\|_{L^{2}(\Omega)}+\|\Psi(\underline{\nabla} \varphi)\|_{L^{2}(\Omega)} \\
& \leq\|\underline{\nabla} \Psi\|_{L^{2}(\Omega)}\|\varphi\|_{L^{\infty}(\Omega)} \\
& \quad+\|\Psi\|_{L^{6}(\Omega)}\|\underline{\nabla} \varphi\|^{1 / 2} L^{2}(\Omega)\|\underline{\nabla} \varphi\|^{1 / 2} L^{6}(\Omega) \\
& \leq C\|\Psi\|_{H^{1}(\Omega)}\|\varphi\|^{1 / 2} H^{1}(\Omega)\|\varphi\|^{1 / 2} H^{2}(\Omega) \\
& \leq C\|\Psi\|^{2} H^{1}(\Omega)\|\varphi\|_{H^{1}(\Omega)}+\epsilon\|\varphi\|_{H^{2}(\Omega)}
\end{aligned}
$$

Proof of the lemma:

That the $\Phi_{\lambda}$ are bounded above follows directly from Theorem 1 and the boundedness of the trace operators. The second part of the technical lemma 
shows that the bound is proportional to $1+\lambda+\|\underline{\gamma}\|^{2} H^{1}(\Omega) \leq 2+\|\underline{\gamma}\|^{2} H^{1}(\Omega)$.

Multiplying (4.1a) by $u$ and integrating over $\Omega \times \widetilde{\jmath}, \tilde{J}=(0, \tau]<J$, yields

$$
\begin{aligned}
& 1 / 2(\phi u, u)(\tau)+\lambda \int_{0}^{\tau}\left(F_{m}\left(u_{t}\right), u\right) d t+\int_{0}^{\tau} B(u, u) d t \\
&=\int_{0}^{\tau}(F, u) d t+\int_{0}^{\tau} \int_{\partial \Omega} u_{0} u d s d t+\int_{0}^{\tau}(\underline{\gamma} u, \underline{\nabla u}) d t \\
& \quad+1 /{ }_{2}\left(\phi u^{0}, u^{0}\right) \\
& \leq C\left\{\|F\|^{2} L^{2}\left(\widetilde{J} ; L^{2}(\Omega)\right)+\left\|u_{0}\right\|^{2} H^{1 / 2}(\partial \Omega)+\left\|u^{0}\right\|^{2} L^{2}(\Omega)\right. \\
&\left.\quad+\int_{0}^{\tau}(\phi u, u) d t\right\}+1 / 2 \int_{0}^{\tau} B(u, u) d t,
\end{aligned}
$$

since the $H^{1 / 2}(\partial \Omega)$-norm of the trace of $u$ is bounded by the $H^{\prime}(\Omega)$-norm of $u$. The first part of the technical lemma shows that $C$ depends on the $H^{\prime}(\Omega)$-norm of $\underline{\gamma}$. By (3.5), we may simply omit the matrix source term on the far left side of (4.6) with respect to the inequality. Then, Gronwall's inequality gives us

$$
\begin{aligned}
& \|U\|^{2} L^{\infty}\left(J: L^{2}(\Omega)\right)+\|\underline{\nabla} U\|^{2} L^{2}\left(J ; L^{2}(\Omega)\right) \\
& \leq C\left\{\|F\|^{2} L^{2}\left(J ; L^{2}(\Omega)\right)+\left\|U_{0}\right\|^{2} H^{1 / 2}(\partial \Omega)+\left\|U^{0}\right\|^{2} L^{2}(\Omega)\right\} \text {. }
\end{aligned}
$$

Next, multiplying (4.1a) by $u_{t}$ and integrating over $\Omega \times \widetilde{J}$ yields

$$
\begin{aligned}
& \int_{0}^{\tau}\left(\phi u_{t}, u_{t}\right) d t+\lambda \int_{0}^{\tau}\left(F_{m}\left(u_{t}\right), u_{t}\right) d t+1 / 2 B(u, u)(\tau) \\
&=\int_{0}^{\tau}\left(F, u_{t}\right) d t+\int_{0}^{\tau} \int_{\partial \Omega} u_{0} u_{t} d s d t+\int_{0}^{\tau}\left(\underline{\gamma} u, \underline{\nabla} u_{t}\right) d t \\
&+1 / 2 B\left(u^{0}, u^{0}\right) .
\end{aligned}
$$

Integrating in time straightforwardly and by parts gives

$$
\begin{aligned}
\int_{0}^{\tau} \int \partial \Omega & u_{0} u_{t} d s d t+\int_{0}^{\tau}\left(\underline{\gamma} u, \underline{\nabla} u_{t}\right) d t \\
=\int \partial \Omega & u_{0}\left[u(\underline{x}, \tau)-u^{0}\right] d s \\
& +(\underline{\gamma} u, \underline{\nabla} u)(\tau)-\left(\underline{\gamma} u^{0}, \underline{\nabla} u^{0}\right)-\int_{0}^{\tau}\left(\underline{\gamma} u_{t}, \underline{\nabla} u\right) d t \\
\leq C\{ & \left\|u_{0}\right\|^{2} H^{1 / 2}(\partial \Omega)+\left\|u^{0}\right\|^{2} H^{1}(\Omega) \\
& \left.+\|u\|^{2} L^{\infty}\left(\tilde{J}: L^{2}(\Omega)\right)+\|\underline{\nabla} u\|^{2} L^{2}\left(\widetilde{J} ; L^{2}(\Omega)\right)\right\} \\
& +1 /{ }_{4} B(u, u)(\tau)+1 / 4 \int_{0}^{\tau}\left(\phi u_{t}, u_{t}\right) d t+\epsilon\|\underline{\nabla} u\|^{2} L^{2}\left(\widetilde{J} ; H^{1}(\Omega)\right) .
\end{aligned}
$$

where $\epsilon$ is as small as we like and, again, $c$ depends on $\|\underline{\gamma}\|_{H^{\prime}(\Omega)}$ by the technical lemma. Combining (4.8) with (3.4), (4.9), and (4.7), we see 


$$
\begin{aligned}
& 1 / 2 \int_{0}^{\tau}\left(\phi u_{t}, u_{t}\right) d t+1 / 4 B(u, u)(\tau) \\
& \leq C\left\{\|F\|^{2} L^{2}\left(J: L^{2}(\Omega)\right)+\left\|u_{0}\right\|^{2} H^{1 / 2}(\partial \Omega)+\left\|u^{0}\right\|^{2} H^{1}(\Omega)\right\} \\
& \quad+\epsilon\|u\|^{2} L^{2}\left(J ; H^{2}(\Omega)\right) .
\end{aligned}
$$

Next, from (4.1a) itself, Theorem 1, and the technical lemma,

$\|\underline{\nabla} \cdot \mathbf{x} \underline{\nabla} u\|_{L^{2}\left(J ; L^{2}(\Omega)\right)}$

$$
\begin{aligned}
& \leq C\left\{(1+\lambda)\left\|u_{t}\right\|_{L^{2}\left(J ; L^{2}(\Omega)\right)}+\|u\|_{L^{2}\left(J ; H^{1}(\Omega)\right)}+\|F\|_{L^{2}\left(J ; L^{2}(\Omega)\right)}\right\} \\
& \quad+\epsilon\|u\|_{L^{2}\left(J: H^{2}(\Omega)\right)}
\end{aligned}
$$

So, for $\epsilon$ having been chosen small enough above, (4.11) combines with elliptic regularity, (4.10), and (4.7) to show that

$$
\begin{aligned}
& \left.\|u\|_{L^{2}\left(J ; H^{2}(\Omega)\right.} \leq \mathrm{Cl}\|\underline{\nabla} \cdot \mathbf{x} \underline{\nabla}\|_{L^{2}\left(J ; L^{2}(\Omega)\right)}+\|u\|_{L^{2}\left(J ; H^{1}(\Omega)\right)}\right\} \\
& \leq C\left\{\|F\|^{2} L^{2}\left(J ; L^{2}(\Omega)\right)+\left\|u_{0}\right\|^{2} H^{1 / 2}(\partial \Omega)+\left\|U^{0}\right\|^{2} H^{1}(\Omega)\right\} .
\end{aligned}
$$

Finally, (4.12) can be used to complete the estimate (4.10) and finish the proof.

Since $\Phi_{\lambda}=\lambda \Phi_{1}+(1-\lambda) \Phi_{0}$, we can now use the method of continuity to deform the solution $\tilde{u}$ of $\Phi_{0}(\tilde{u})=\left(F, U_{0}, u^{0}\right)$ continuously to a solution $u$ of $\Phi_{1}(u)=\left(F, u_{0}, u^{0}\right)$. That is, from the lemma, if $\Phi_{\lambda_{0}}$ is an isomorphism, then $\Psi\left(u ; \lambda, \lambda_{0}\right)=$ $\Phi_{\lambda_{0}}{ }^{-1}\left(\left(F, u_{0}, u^{0}\right)+\left(\Phi_{\lambda_{0}}-\Phi_{\lambda}\right)(u)\right)$ is a contraction mapping on $\boldsymbol{v}$ for $\left|\lambda-\lambda_{0}\right|$ small enough independently of $\lambda$ and $\lambda_{0}$. The fixed point of $\Psi$ is the solution to (4.1), so $\Phi_{\lambda}$ is also an isomorphism. After a finite number of steps from $\lambda_{0}=0$, we have:

\section{Corollary:}

$\Phi_{1}$ is an isomorphism.

\section{Theorem 3:}

If $f \in L^{2}\left(J ; L^{2}(\Omega)\right)$ and $\left(\rho_{0}\right)^{2}, \rho^{0} \in H^{1}(\Omega)$ are such that $\left[k \underline{\nabla} \rho^{0}-\underline{\gamma}\left(\rho^{0}-1 / 2 \rho_{0}\right)\right] \cdot \underline{v}=0$ on $\partial \Omega$, then the double porosity model (1.4) through (1.7) has a unique solution $\left(\rho, \rho_{m}\right) \in \boldsymbol{V} \times \boldsymbol{v}_{m}, \boldsymbol{v}_{m}=H^{1}\left(J ; L^{2}\left(\Omega_{m}\right)\right) \cap L^{2}\left(J ; H^{2}\left(\Omega_{m}\right)\right)$, which varies continuously with the data: 
$\|\rho\|_{H^{\prime}\left(J ; L^{2}(\Omega)\right)}+\|\rho\|_{L^{2}\left(J: H^{2}(\Omega)\right)}+\left\|\rho_{m}\right\|_{H^{\prime}\left(J: L^{2}\left(\Omega_{m}\right)\right)}$

$$
\begin{gathered}
+\left\|\rho_{m}\right\|_{L^{2}\left(J ; H^{2}\left(\Omega_{m}\right)\right)} \\
\leq C\left\{\|f\|_{L^{2}\left(J ; L^{2}(\Omega)\right)}+\left\|\left(\rho_{0}\right)^{2}\right\|_{H^{\prime}(\Omega)}+\left\|\rho^{0}\right\|_{H^{\prime}(\Omega)}\right\},
\end{gathered}
$$

where $C$ depends on $\left\|\rho_{0}\right\|_{H^{\prime}}(\Omega)$ and, for solutions $\left(\rho_{j}, \rho_{m, j}\right)$ arising from data $\left(f_{j}, \rho_{0, j}, \rho^{0}{ }_{j}\right), j=1$ and 2 ,

(4.14)

$$
\begin{aligned}
& \left\|\rho_{1}-\rho_{2}\right\|_{H^{\prime}\left(J ; L^{2}(\Omega)\right)}+\left\|\rho_{1}-\rho_{2}\right\|_{L^{2}\left(J ; H^{2}(\Omega)\right)} \\
& +\left\|\rho_{m, 1}-\rho_{m, 2}\right\|_{H^{\prime}\left(J ; L^{2}\left(\Omega_{m}\right)\right)}+\left\|\rho_{m, 1}-\rho_{m, 2}\right\|_{L^{2}\left(J ; H^{2}\left(\Omega_{m}\right)\right)} \\
& \leq C\left\{\left\|f_{1}-f_{2}\right\|_{L^{2}\left(J ; L^{2}(\Omega)\right)}+\left\|\left(\rho_{0,1}\right)^{2}-\left(\rho_{0,2}\right)^{2}\right\|_{H^{\prime}(\Omega)}\right. \\
& \left.+\left\|\rho_{0,1}-p_{0,2}\right\|_{H^{\prime}(\Omega)}+\left\|\rho_{1}^{0}-\rho_{2}^{0}\right\|_{H^{\prime}(\Omega)}\right\},
\end{aligned}
$$

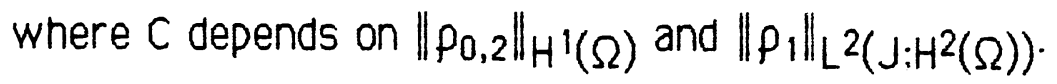

Note that Hölder's inequality and the Sobolev imbedding theorem imply that

$$
\|\Psi \varphi\|_{H^{\prime}(\Omega)} \leq C\|\Psi\|_{W^{1.12 / 5}(\Omega)}\|\varphi\|_{W^{1,12 / 5}(\Omega)}
$$

Hence, we can replace the norm of $\left(\rho_{0}\right)^{2}$ in (4.13) by $\left\|\rho_{0}\right\|^{2} w^{1,12 / 5}(\Omega)$, and we can replace the norms involving $\rho_{0,1}$ and $\rho_{0,2}$ in (4.14) by $\left(1+\left\|\rho_{0,1}\right\|_{W} 1,12 / 5(\Omega)\right.$ $\left.+\left\|\rho_{0,2}\right\|_{W^{1,12 / 5}(\Omega)}\right)\left\|\rho_{0,1}-\rho_{0,2}\right\|_{W^{1,12 / 5}(\Omega)}$

Proof:

The claims for $\rho$ and $\rho_{1}-\rho_{2}$ follow directly from the remarks following (4.1) and the lemma with its corollary. Now, since $\rho$ exists and is unique, $P_{m}$ exists and is unique. If we finish the energy estimates (3.6) and (3.9) (with $u_{*}=\rho_{t}$ and $u=\rho$ so that $\rho_{m}=v+\hat{\rho}^{0}{ }_{j}$ ), and if we use (1.6a) itself and elliptic regularity, we can bound $P_{m}$ in $H^{1}\left(J ; L^{2}\left(\Omega_{m}\right)\right)$ and in $L^{2}\left(J ; H^{2}\left(\Omega_{m}\right)\right)$ by

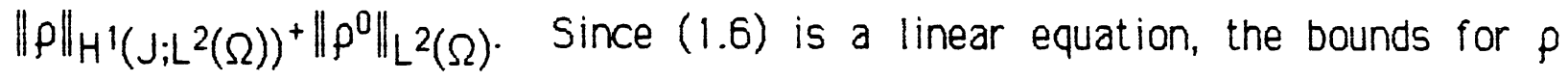
finish the proof.

Later we will want to take higher Sobolev norms of $\rho$ and $\rho_{m}$. so it is appropriate at least to say something about their smoothness here. From (1.6a) 
we see that $\rho_{m}$ is inf initely differentiable on $\Omega_{m} \times J$. Then (2.1) and (1.5b) tell us that $f_{m}$ is infinitely differentiable in time and as smooth in space as the $X_{i}$ are. Finally, then, (1.4a) tells us (see [4], Chapter 3, Theorem 11, p. 74)

Theorem 4:

If each of $\partial^{|\alpha|} x_{i} / \partial x^{\alpha}, \partial^{|\alpha|+j}\left[f+1 / 2 \underline{\nabla} \cdot\left(p_{0} \underline{\gamma}\right)\right] / \partial x^{\alpha} \partial t j, \partial^{|\alpha|} \mid \phi / \partial x^{\alpha}, \partial^{|\alpha|} x / \partial x^{\alpha}$, $\partial^{|\alpha|}(\underline{\gamma}-\underline{\nabla} \cdot x) / \partial x^{\alpha}$, and $\partial^{|\alpha|}(\underline{\nabla} \cdot \underline{\gamma}) / \partial x^{\alpha}$ are Hölder continuous of order $\beta \epsilon(0,1)$ on $\Omega$ or $\Omega \times J$, as appropriate, for $0 \leq|\alpha|+2 j \leq l$ and $0 \leq j \leq m$ (where $\alpha$ is a multi-index), then $\partial^{|\alpha|+j} \rho / \partial x^{\alpha} \partial t j$ is Hölder continuous of order $\beta$ on $\Omega \times J$ for $0 \leqslant|\alpha|+2 j \leq l+2$ and $0 \leq j \leq m+1$.

\section{Formulation of the finite element method.}

For each $h$ and $h_{m}$ in $(0,1]$, let $\mathbf{M C H}(\Omega)$ and, for each $i, \mathbf{N}_{i} C H_{0}^{1}\left(\Omega_{j}\right)$ be standard Galerkin finite dimensional $\mathrm{H}^{\prime}$-approximation spaces of order $\mathrm{r}$ in $\mathrm{h}$ and $s$ in $h_{m}$, respectively. That is, let

$$
\inf _{\varphi \in \mathcal{M}}\|u-\varphi\|_{H} j(\Omega) \leq C\left\|_{u}\right\|_{H} P(\Omega)^{h P-j}, \quad 1 \leq p \leq r, j=-1,0,1 ;
$$

for any $\mathrm{uH}^{\mathrm{r}}(\Omega)$ and, for each i,

$$
\text { inf }\|v-\psi\|_{H} j\left(\Omega_{i}\right) \leq C\|v\|_{H} q\left(\Omega_{i}\right)^{h_{m}}{ }^{q-j}, \quad 1 \leq q \leq s, j=-1,0,1
$$

$$
\Psi \in \mathbf{N}_{\mathrm{i}}
$$

for any $v \in H^{S}\left(\Omega_{i}\right) \cap H_{0}{ }_{0}\left(\Omega_{j}\right)$ (where the constants $C$ are independent of $u, v, h$, and $\left.h_{m}\right)$. To avoid trivialities below, we will assume that $\boldsymbol{M}$ contains the constant functions. We will also let $\mathbf{N}_{i}^{*}=\left\{v^{*}=v+c: v \in \mathbf{N}_{j}\right.$ and $\left.c \in \mathbb{R}\right\}$. In both (5.1) and (5.2), the result for $\mathrm{j}=1$ implies the result for $\mathrm{j}=-1$ and $\mathrm{j}=0$ (see (6.6) and (6.7) Delow).

For $u \in H^{1}(\Omega)$, let $u^{\prime} \in \boldsymbol{M}$ denote the elliptic projection defined by

$$
B\left(u-u^{\prime}, \varphi\right)=0 \quad \text { for all } \varphi \in \boldsymbol{M}
$$

and

(5.3b) $\quad \int_{\Omega}\left(u-u^{\prime}\right) d x=0$. 
For each positive integer $N$, let $\Delta t=T / N$. We will use the following notation (where $u$ is any function of time):

$$
t_{n}=n \Delta t
$$

and

$$
u^{n}=u\left(t_{n}\right)
$$

for $n=0,1, \ldots, N$, and

$$
u^{n-1 / 2}=\frac{u^{n}+u^{n-1}}{2}
$$

and

$$
\partial u^{n}=\frac{u^{n}-u^{n-1}}{\Delta t}
$$

for $n=1,2, \ldots, N$.

We shall now describe our finite element procedure. We seek a map $U:\left\{t_{0}\right.$, $\left.t_{1}, \ldots, t_{N}\right\} \rightarrow M$ and, on each $\Omega_{i}$, a map $W:\left\{t_{1}, t_{2}, \ldots, t_{N}\right\} \rightarrow N_{i}^{*}$ and a function $Z \in \mathbb{N}_{i}^{*}$ that satisfy the following equations:

$(5.4 a)$

$$
\left(\phi \partial U^{n}, \varphi\right)+\sum_{i} \phi_{m i} \partial \bar{V}_{i}^{n} \hat{\varphi}_{i}\left|\Omega_{i}\right|+B\left(U^{n-1 / 2}, \varphi\right)-\left(\underline{\gamma} u^{n-1 / 2}, \underline{\nabla} \varphi\right)
$$

(5.4D) $\quad U^{0}=\rho^{0}$,

$(5.5 a)$

$$
=\left(f^{n-1 / 2}, \varphi\right)-1 / 2\left(\underline{\gamma} \rho_{0}, \underline{\nabla} \varphi\right) \quad \text { for } \varphi \in \boldsymbol{M} .
$$

$(5.5 b)$

$$
\phi_{m i}\left[\frac{w^{n}-v^{n-1}}{\Delta t}, \psi\right]_{i}+B_{i}\left[\frac{w^{n}+v^{n-1}}{2}, \psi\right]=0 \quad \text { for } \psi \in N_{i}
$$

$$
w^{n}=\hat{U}_{i}^{n-1} \quad \text { on } \partial \Omega_{i} \text {, }
$$

$$
\phi_{m i}\left[\frac{Z}{\Delta t}, \psi\right]_{i}+B_{i}\left[\frac{Z}{2}, \psi\right]=0 \quad \text { for } \psi \in \mathbb{N}_{i}
$$

(5.6b) $\quad z=\Delta t \quad$ on $\partial \Omega_{j}$, 
$(5.7 a)$

$$
v^{n}=w^{n}+\partial \hat{U}_{i}^{n} z \quad \text { on } \Omega_{i}
$$

and

$$
V^{0}=\hat{U}_{i}^{0}
$$

First, we solve (5.4b) for $U^{0}$ and (5.6) for $Z$. Then, successively for $n=1,2, \ldots$, $N$, we solve (5.5) for $w^{n},(5.7 a)$ for $V^{n}$ (with its explicit dependence on $U^{n}$ ), (5.4a) for $U^{n}$, and, finally, we return to (5.7a) to remove the $U^{n}$ dependence from $v^{n}$. Since uniqueness implies existence, it is not terribly hard to see that the equations can indeed be solved, provided that, if gravity is present, $\Delta t$ is not too large. This, in fact, follows from the analysis below.

It will be shown in the next section that $U$ and $V$ approximate $\rho$ and $\rho_{m}$. respectively.

Because of the enormous number of matrix blocks, the approximation procedure appears at first glance to require a massive amount of calculation. However, in actual reservoir simulation, $h$ is of necessity taken to be much larger than the diameters of the matrix blocks; consequently, the triangulation over each matrix block should be rather coarse (see Theorem 5 below). and thus the linear systems arising from (5.5) and (5.6) should be small. Since one is generally only interested in effects that are on the scale of the discretization of the fracture system, it is sufficient to solve (5.5) and (5.6) only on the blocks that sit over the quadrature points of (5.4a). If $\Omega_{j}$ is symmetric about its center, the solution $V$ is also symmetric over $\Omega_{j}$, so, with some care, one can further reduce the size of the linear systems in (5.5) and (5.6). Finally, the block problems are independent of each other by assumption 3, so naturally fractured reservoir simulation (and, in particular, our finite element procedure) is well suited to parallel type computing machines.

\section{Analysis of the approximation error.}

Let us define the error functions

$$
\zeta=\rho-U
$$

and

$$
\xi=\rho_{m}-V .
$$


Subtract (5.4a) from the average of (2.2) at times $t_{n}$ and $t_{n-1}$ to obtain the error equation

$$
\begin{array}{r}
\left(\phi \partial \zeta^{n}, \varphi\right)+\sum_{i} \phi_{m i} \partial \bar{\xi}_{i}^{n} \hat{\varphi}_{i}\left|\Omega_{i}\right|+B\left(\zeta^{n-1 / 2}, \varphi\right)-\left(\underline{\gamma} \zeta^{n-1 / 2}, \underline{\nabla} \varphi\right) \\
=\left(\phi\left(\partial \rho^{n}-\rho_{t}^{n-1 / 2}\right), \varphi\right)+\sum_{i} \phi_{m i}\left(\partial \bar{\rho}_{m}, i^{n}-\bar{\rho}_{m}, t, i^{n-1 / 2}\right) \hat{\varphi}_{i}\left|\Omega_{i}\right| \\
\text { for } \varphi \in \mathbf{M},
\end{array}
$$

$$
\zeta^{0}=\rho^{0}-\rho^{0} .
$$

Now subtract the sum of (5.5a) and $\partial \hat{U}_{i}^{n}$ times (5.6a) from the average of (2.3) at times $t_{n}$ and $t_{n-1}$ to obtain the other error equation

$$
\begin{aligned}
& \phi_{m i}\left(\partial \xi^{n}, \psi\right)_{i}+B_{i}\left(\xi^{n-1 / 2}, \psi\right) \\
& =\phi_{m i}\left(\partial \rho_{m}{ }^{n}-\rho_{m, t} t^{n-1 / 2}, \psi\right)_{i} \quad \text { for } \psi \in N_{i},
\end{aligned}
$$

$$
\xi^{n}=\hat{\zeta}_{i}^{n} \quad \text { on } \partial \Omega_{i} \text {, }
$$

$$
\xi^{0}=\hat{\zeta}_{i}^{0}
$$

Note [12] that $\zeta=\rho^{-} \rho^{\prime}+\rho^{\prime}-U=\left(\rho^{-} \rho^{\prime}\right)+\zeta^{\prime}$. Similarly, we can write $\xi=\left(\rho_{m}\right.$ $\left.-\rho_{m}^{\prime \prime}\right)+\xi^{\prime \prime}$. where we extend the following definition to all of $\Omega_{m}$ : for any $v \in H^{1}\left(\Omega_{\mathrm{i}}\right)$ that is constant on $\partial \Omega_{\mathrm{i}}, V^{\prime \prime} \in \mathbf{N}_{\mathrm{i}}{ }^{*}$ is the elliptic projection given by

$$
B_{i}\left(v-v^{\prime \prime}, \Psi\right)=0 \quad \text { for all } \Psi \in \mathbf{N}_{i}
$$

and

$$
v^{\prime \prime}=v \quad \text { on } \partial \Omega_{i}
$$

Elliptic theory tells us that

$$
\begin{aligned}
\left\|\partial^{k}\left(\rho-\rho^{\prime}\right) / \partial t^{k}\right\|_{H} J(\Omega) \leq C\left\|\partial^{k} \rho / \partial t^{k}\right\|_{H P(\Omega)} h^{P^{-j}} ; \\
\qquad 1 \leq p \leq r ; j=-1,0 ; k=0,1 ;
\end{aligned}
$$

$$
\left\|\underline{\nabla}\left(\rho-\rho^{\prime}\right)\right\|_{L^{2}(\Omega)} \leq C\|\rho\|_{H} P(\Omega)^{h^{P-1}}, \quad 1 \leq p \leq r ;
$$




$$
\begin{array}{r}
\left\|\partial^{k}\left(\rho_{m}-\rho_{m}{ }^{\prime)}\right) / \partial t^{k}\right\|_{H} j\left(\Omega_{m}\right) \leq C\left\|\partial^{k} \rho_{m} / \partial t^{k}\right\|_{H} q\left(\Omega_{m}\right)^{n_{m}}{ }^{q-j}, \\
1 \leq q \leq s ; j=-1,0 ; k=0,1 ;
\end{array}
$$

and

(6.7b) $\quad\left\|\underline{\nabla}\left(\rho_{m}-\rho_{m}{ }^{\prime \prime}\right)\right\|_{L}{ }^{2}\left(\Omega_{m}\right) \leq C\left\|\rho_{m}\right\|_{H} q\left(\Omega_{m}\right)^{n_{m}}{ }^{q-1}, \quad 1 \leq q \leq s$.

In deriving (6.6a), the standard Nitsche duality argument [7] applies, as the smoothness of $\partial \Omega$ ensures the regularity of the dual problem. In deriving (6.7a), since $\Omega_{i}$ is convex, the dual problem is regular enough when $j=0$, but not necessarily so when $j=-1$. However, in this case, the duality argument can still be performed by posing the dual problem on a larger domain $\Omega_{i}^{*}$ with smooth boundary via the (bounded) Calderón extension operator $E: H^{\prime}\left(\Omega_{i}\right) \rightarrow H^{1}\left(\Omega_{j}^{*}\right)$ [2] as

$$
-\underline{\nabla} \cdot\left[\left(E x_{m}\right) \underline{\nabla} \varphi\right]=E \Psi, \quad \text { on } \Omega_{i}^{*}
$$

$$
\varphi=0, \quad \text { on } \partial \Omega_{i}^{*},
$$

to obtain the needed regularity:

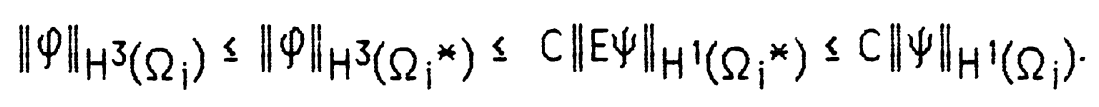

The boundary term from the integration by parts, which is normally zero by the dual problem, is now zero simply because $\rho_{m}-\rho_{m}{ }^{\prime \prime}=0$ on $\partial \Omega_{\mathrm{i}}$.

It remains to estimate the errors ' $\zeta^{\prime}$ and $\xi "$. Rewrite the error equations (6.3) and (6.4) in terms of these elliptic projections:

(6.10a)

$$
\begin{aligned}
& \left(\phi \partial \zeta^{\prime n}, \varphi\right)+\sum_{i} \phi_{m i} \partial \bar{\xi}^{\prime \prime}{ }_{i}^{n} \hat{\varphi}_{i}\left|\Omega_{i}\right|+B\left(\zeta^{n-1 / 2}, \varphi\right)-\left(\underline{\gamma} \zeta^{, n-1 / 2}, \underline{\nabla} \varphi\right) \\
& =\left(\phi\left(\partial \rho^{n}-\rho_{t}^{n-1 / 2}\right), \varphi\right)+\sum_{i} \phi_{m i}\left(\partial \bar{\rho}_{m}{ }^{n} i^{n}-\bar{\rho}_{m, t, i}{ }^{n-1 / 2}\right) \hat{\varphi}_{i}\left|\Omega_{i}\right| \\
& -\left(\underline{\gamma}\left(\rho^{n-1 / 2}-\rho^{n-1 / 2}\right), \underline{\nabla} \varphi\right) \quad \text { for } \varphi \in \mathbf{M} \text {. }
\end{aligned}
$$

(6.10b) $\quad \zeta^{\circ}=0$, 
$(6.11 a)$

$$
\phi_{m i}\left(\partial \xi^{n n}, \psi\right)_{i}+B_{i}\left(\xi^{n n-1 / 2}, \psi\right)
$$

$$
=\phi_{m i}\left(\partial \rho_{m}{ }^{\prime n}-\rho_{m, t}{ }^{n-1 / 2}, \psi\right)_{i} \quad \text { for } \psi \in N_{i} \text {, }
$$

(6.11b)

$$
\xi^{\prime \prime}=\hat{\zeta}_{i}^{n}-\left(\hat{\rho}_{i}^{\prime}{ }^{n}-\hat{\rho}_{i}^{n}\right) \quad \text { on } \partial \Omega_{i} \text {, }
$$

$(6.11 c)$

$$
\xi^{\prime \prime 0}=-\left(\hat{\rho}^{0}{ }_{i}-\hat{\rho}_{i}^{0}\right) .
$$

It is well known how to analyze every term above ([3] and [12]) except those that represent the matrix source. We will combine these known ideas with generalizations of the ideas presented in sections 3 and 4 to accomplish our error analysis.

Let

$$
\begin{aligned}
& E_{1}(P, q, j)=\left\{\|\rho\|_{L^{\infty}\left(J ; H^{P+}(\Omega)\right)} h^{j-1}+\left\|\rho_{t}\right\|_{L^{2}(J ; H P(\Omega))}\right\} h^{P+1}
\end{aligned}
$$

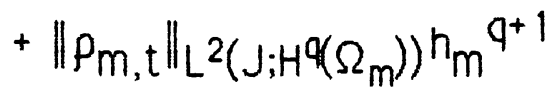

$$
\begin{aligned}
& +\left\{\left\|\partial^{3} \rho / \partial t^{3}\right\|_{L^{2}\left(J ; H^{-1}(\Omega)\right)}+\left\|\partial^{3} P_{m} / \partial t^{3}\right\|_{L^{2}\left(J ; H^{-1}\left(\Omega_{m}\right)\right)}\right\}(\Delta t)^{2}
\end{aligned}
$$

where, always, $1 \leq p \leq r-j, 1 \leq q \leq 5$, and $j=0$ or 1 . Note that

$$
\begin{aligned}
& \text { N } \\
& \sum\left\{\left\|\partial \rho^{\prime n}-\rho_{t}{ }^{n-1 / 2}\right\|^{2} H^{-1}(\Omega)+\left\|\partial \rho_{m}{ }^{n}-\rho_{m}, t^{n-1 / 2}\right\|^{2} H^{-1}\left(\Omega_{m}\right)\right. \\
& n=1 \\
& \left.+\sum_{i}\left[\left(\partial \hat{\rho}_{i}^{\prime}{ }^{n}-\partial \hat{\rho}_{i}{ }^{n}\right)^{2}+\left(\partial \bar{\rho}_{m}{ }^{\prime \prime} i^{n}-\bar{\rho}_{m, t, i} i^{n-1 / 2}\right)^{2}\right]\right\} \Delta t \\
& +\max _{0 \leq n \leq N}\left\|\rho^{n}-\rho^{n}\right\|^{2} L^{2}(\Omega) \\
& \leq C E_{1}^{2}(p, q, j) \text {. }
\end{aligned}
$$

In obtaining the optimal bound on the third term on the left side above, we have used that $\chi_{i} \in H^{\prime}(\Omega)$, for all i.

First, take $\psi=\xi^{\prime \prime n-1 / 2}-\left[\hat{\zeta}_{i}^{n-1 / 2}-\left(\hat{\rho}^{\prime} i^{n-1 / 2}-\hat{p}_{i}^{n-1 / 2}\right)\right]$ in (6.11a). After manipulating, summing on $n$ from 1 to $\eta, \eta \leq N$, and summing on $i$, we see 


$$
\begin{aligned}
& 1 / 2\left(\phi_{m} \xi^{\prime \prime \eta} \cdot \xi^{\prime \prime} \eta\right)_{m}-\sum_{n=1}^{\eta} \sum_{i} \phi_{m i} \partial \overline{\xi^{\prime \prime}}{ }_{i}{ }^{n} \hat{\zeta}_{i}{ }^{n-1 / 2}\left|\Omega_{i}\right| \Delta t \\
& +\sum_{n=1}^{\eta} B_{m}\left(\xi^{n-1 / 2}, \xi^{n n-1 / 2}\right) \Delta t \\
& =1 / 2\left(\phi_{m} \xi^{n 0}, \xi^{\prime \prime 0}\right)_{m} \\
& -\sum_{n=1}^{\eta} \sum_{i} \phi_{m i} \partial \bar{\xi}^{\prime \prime} i^{n}\left(\hat{p}_{i}{ }^{n-1 / 2}-\hat{p}_{i}^{n-1 / 2}\right)\left|\Omega_{i}\right| \Delta t \\
& +\sum_{n=1}^{\eta}\left\{\left(\phi_{m}\left(\partial \rho_{m} n-\rho_{m}, t^{n-1 / 2}\right), \xi^{n n-1 / 2}\right)_{m}\right. \\
& \begin{aligned}
-\sum_{i} \phi_{m i}\left(\partial \bar{\rho}_{m}{ }^{n}{ }^{n}-\bar{\rho}_{m, t, i} i^{n-1 / 2}\right)\left[\hat{\rho}_{i}^{n-1 / 2}\right. & \\
& \left.\left.-\left(\hat{\rho}_{i}^{\prime}{ }^{n-1 / 2}-\hat{\rho}_{i}^{n-1 / 2}\right)\right]\left|\Omega_{i}\right|\right\} \Delta t
\end{aligned}
\end{aligned}
$$

$$
\begin{aligned}
& =-1 / 2 \sum_{i} \phi_{m i}\left(\hat{\rho}^{0_{i}}-\hat{\rho}_{i}^{0}\right)^{2}\left|\Omega_{i}\right| \\
& -\sum_{i} \phi_{m i} \bar{\xi}_{i}^{\prime \prime} \eta\left(\hat{\rho}_{i}^{\prime} \eta-\hat{\rho}_{i} \eta\right)\left|\Omega_{i}\right| \\
& +\sum_{n=1}^{\eta} \sum_{i} \phi_{m i} \bar{\xi}_{i}^{n} i^{n-1 / 2}\left(\partial \hat{\rho}_{i}^{\prime}{ }^{n}-\partial \hat{\rho}_{i}^{n}\right)\left|\Omega_{i}\right| \Delta t \\
& +\sum_{n=1}^{\eta}\left\{\left(\phi_{m}\left(\partial \rho_{m}{ }^{\prime n}-\rho_{m}, t^{n-1 / 2}\right), \xi^{n n-1 / 2}\right)_{m}\right. \\
& -\sum_{i} \phi_{m i}\left(\partial \bar{\rho}_{m}{ }^{\prime \prime}{ }^{n}-\bar{\rho}_{m, t, i} i^{n-1 / 2}\right)\left[\hat{\zeta}_{i}^{n} i^{n-1 / 2}\right. \\
& \left.\left.-\left(\hat{\rho}_{i}^{\prime} i^{n-1 / 2}-\hat{\rho}_{i}^{n-1 / 2}\right)\right]\left|\Omega_{i}\right|\right\} \Delta t
\end{aligned}
$$

$$
\begin{aligned}
& \leq C E_{1}^{2}(p, q, j)+\epsilon\left\{\sum_{n=1}^{\eta}\left(\phi_{m} \xi^{m n-1 / 2}, \xi^{n n-1 / 2}\right)_{m} \Delta t\right. \\
& \left.+\sum_{n=1}^{\eta}\left(\phi \zeta^{n-1 / 2}, \zeta^{n-1 / 2}\right) \Delta t\right\} \\
& +1 / 4\left(\phi_{m} \xi^{n \eta \eta}, \xi^{\prime \prime \eta}\right)_{m}+1 / 2 \sum_{n=1}^{\eta} B_{m}\left(\xi^{n n-1 / 2}, \xi^{n-1 / 2}\right) \Delta t
\end{aligned}
$$


where we have used summation by parts in the second equality above.

Next, estimate the sum on $n$ of (6.10a) with $\varphi=\zeta^{n-1 / 2}$ to see

$$
\begin{aligned}
& 1 / 2\left(\phi \zeta^{\prime} \eta, \zeta^{\prime} \eta\right)+\sum^{\eta} \sum \phi_{m i} \partial \bar{\xi}_{i}^{\prime \prime}{ }_{i}^{n} \hat{\zeta}_{i}^{\prime n-1 / 2}\left|\Omega_{i}\right| \Delta t \\
& n=1 \text { i } \\
& +\sum_{n=1}^{\eta} B\left(\zeta^{n-1 / 2}, \zeta^{, n-1 / 2}\right) \Delta t \\
& =\sum_{n=1}^{\eta}\left(\underline{\gamma} \zeta^{n-1 / 2}, \underline{\nabla} \zeta^{n-1 / 2}\right) \Delta t \\
& +\sum_{n=1}^{\eta}\left\{\left(\phi\left(\partial \rho^{\prime n}-\rho t^{n-1 / 2}\right), \zeta^{n-1 / 2}\right)\right. \\
& +\sum_{i} \phi_{m i}\left(\partial \bar{\rho}_{m}{ }_{i}^{n}-\bar{\rho}_{m, t, i}{ }^{n-1 / 2}\right) \hat{\zeta}_{i}^{n-1 / 2}\left|\Omega_{i}\right| \\
& \left.-\left(\underline{\gamma}\left(\rho^{n-1 / 2}-\rho^{n-1 / 2}\right), \underline{\nabla} \zeta^{\cdot n-1 / 2}\right)\right\} \Delta t \\
& \leq C\left\{E_{1}{ }^{2}(p, q, j)+\sum_{n=1}^{\eta}\left(\phi \zeta^{n-1 / 2}, \zeta^{n-1 / 2}\right) \Delta t\right\} \\
& +1 / 2 \sum_{n=1}^{\eta} B\left(\zeta^{n-1 / 2} \cdot \zeta^{n-1 / 2}\right) \Delta t .
\end{aligned}
$$

We remove the coupling term between (6.13) and (6.14) by adding the two equations together. With the obvious cancellations, we obtain

$$
\begin{aligned}
& 1 / 2\left(\phi \zeta^{\prime} \eta, \zeta^{\prime} \eta\right)+1 / 2 \sum_{n=1}^{\eta} B\left(\zeta^{\prime n-1 / 2}, \zeta^{n-1 / 2}\right) \Delta t \\
& +1 / 4\left(\phi_{m} \xi^{\prime \prime \eta}, \xi^{\prime \prime \eta}\right)_{m}+1 / 2 \sum_{n=1}^{\eta} B_{m}\left(\xi^{\prime \prime n-1 / 2}, \xi^{\prime n-1 / 2}\right) \Delta t
\end{aligned}
$$

$$
\begin{aligned}
& \leq C\left\{E_{1}^{2}(p, q, j)+\sum_{n=1}^{\eta}\left(\phi \zeta^{, n-1 / 2}, \zeta^{n-1 / 2}\right) \Delta t\right\} \\
&+\epsilon \sum_{n=1}^{\eta}\left(\phi_{m} \xi^{n n-1 / 2}, \xi^{n n-1 / 2}\right)_{m} \Delta t .
\end{aligned}
$$

If $\Delta t$ is not too large, the discrete form of Gronwall's inequality can be applied to $(6.15)$ to yield, after some manipulation, the error estimates 
(6.16)

$$
\max _{0 \leq n \leq N}\left\|\xi^{\prime \prime n}\right\|^{2}{ }^{2}\left(\Omega_{m}\right)+\sum_{n=1}^{N}\left\|\underline{\nabla} \xi^{\prime \prime n}\right\|^{2} L^{2}\left(\Omega_{m}\right) \Delta t \leq C E_{1}{ }^{2}(p, q, j)
$$

and

$$
\max _{0 \leq n \leq N}\left\|\zeta^{\prime n}\right\|^{2} L^{2}(\Omega)+\sum_{n=1}^{N}\left\|\underline{\nabla} \zeta^{\prime n}\right\|^{2} L^{2}(\Omega) \Delta t \leq C E_{1}^{2}(p, q, j) .
$$

For the next set of error estimates, let

$$
\begin{aligned}
E_{2}(P, q)= & \left\{\|p\|_{L^{\infty}(J ; H P(\Omega))}+\left\|p_{t}\right\|_{L^{2}(J ; H P(\Omega))}\right\} h^{P} \\
& \left.+\left\|\rho_{m}, t\right\|_{L^{2}(J ; H} q\left(\Omega_{m}\right)\right)^{h_{m}}{ }^{q} \\
& +\left\{\left\|\partial^{3} \rho / \partial t^{3}\right\|_{L^{2}\left(J ; L^{2}(\Omega)\right)}+\left\|\partial^{3} P_{m} / \partial t^{3}\right\|_{L^{2}\left(J ; L^{2}\left(\Omega_{m}\right)\right)}\right\}(\Delta t)^{2}
\end{aligned}
$$

where $1 \leq p \leq r$ and $1 \leq q \leq 5$. Note that

$$
\begin{aligned}
& \sum_{n=1}^{N}\left\{\left\|\partial \rho^{\prime n}-\rho_{t}^{n-1 / 2}\right\|^{2} L^{2}(\Omega)+\left\|\partial \rho^{\prime n}-\partial \rho^{n}\right\|^{2} L^{2}(\Omega)\right. \\
& \left.+\left\|\partial \rho_{m}{ }^{\prime \prime}-\rho m, t^{n-1 / 2}\right\|^{2} L^{2}\left(\Omega_{m}\right)\right\} \Delta t \\
& \quad+\max _{0 \leq n \leq N}\left\|\rho^{\prime n}-\rho^{n}\right\|^{2} L^{2}(\Omega)+E_{1}{ }^{2}(p, q, 0) \\
& \leq C E_{2}{ }^{2}(p, q) .
\end{aligned}
$$

This time, take $\psi=\partial \xi^{\prime n}-\left[\partial \hat{\zeta}_{i}^{\prime n}-\left(\partial \hat{p}^{\prime}{ }^{n}-\partial \hat{p}_{i}^{n}\right)\right]$ in (6.11a). Again, manipulation and summation on $n$ and $i$ yields

$$
\begin{gathered}
\sum_{n=1}^{\eta}\left(\phi_{m} \partial \xi^{\prime \prime n}, \partial \xi^{\prime \prime n}\right)_{m} \Delta t-\sum_{n=1}^{\eta} \sum_{i} \phi_{m i} \partial \xi^{\prime \prime}{ }_{i}^{n} \partial \hat{\zeta}_{i}^{\prime}{ }^{n}\left|\Omega_{i}\right| \Delta t \\
+1 / 2 B_{m}(\xi " \eta, \xi " \eta)
\end{gathered}
$$


(6.19)

$$
\begin{aligned}
& =-\sum \sum \phi_{m i} \partial \bar{\xi}_{i}^{\prime n}\left(\partial \hat{\rho}_{i}^{\prime}{ }^{n}-\partial \hat{\rho}_{i}^{n}\right)\left|\Omega_{i}\right| \Delta t \\
& n=1 \text { i } \\
& +\sum^{\eta} \sum\left(\phi_{m i}\left(\partial \rho_{m}{ }^{n n}-\rho_{m, t}{ }^{n-1 / 2}\right)\right. \\
& \left.n=1 i \quad \partial \xi^{\prime \prime}-\left[\partial \hat{\zeta}_{i}^{\prime}{ }^{n}-\left(\partial \hat{\rho}_{i}^{\prime} i^{n}-\partial \hat{\rho}_{i}^{n}\right)\right]\right)_{i} \Delta t \\
& \leq C E_{2}^{2}(p, q)+1 / 2 \sum_{n=1}^{\eta}\left(\phi_{m} \partial \xi^{\prime \prime}, \partial \xi^{\prime \prime n}\right)_{m} \Delta t \\
& +1 / 4 \sum_{n=1}^{\eta}\left(\phi \partial \zeta^{\prime n}, \partial \zeta^{\prime n}\right) \Delta t
\end{aligned}
$$

Also, take $\varphi=\partial \zeta^{\prime n}$ in (6.10a) and sum on $n$ to see

$$
\begin{aligned}
& \sum_{n=1}^{\eta}\left(\phi \partial \zeta^{\prime n}, \partial \zeta^{\prime n}\right) \Delta t+\sum_{n=1}^{\eta} \sum_{i} \phi_{m i} \partial \bar{\xi}_{i}^{\prime \prime}{ }^{n} \partial \hat{\zeta}_{i}^{\prime}{ }_{i}^{n}\left|\Omega_{i}\right| \Delta t \\
& +1 /{ }_{2} B\left(\zeta^{\prime} \eta, \zeta^{\prime} \eta\right) \\
& =\sum_{n=1}^{\eta}\left(\underline{\gamma} \zeta^{, n-1 / 2}, \underline{\nabla} \partial \zeta^{, n}\right) \Delta t \\
& +\sum_{n=1}^{\eta}\left\{\left(\phi\left(\partial \rho^{n}-\rho_{t}^{n-1 / 2}\right), \partial \zeta^{n}\right)\right. \\
& +\sum_{i} \phi_{m i}\left(\partial \bar{\rho}_{m}{ }_{i}{ }^{n}-\bar{\rho}_{m, t, i}{ }^{n-1 / 2}\right) \partial \hat{\zeta}_{i}^{\prime}{ }^{n}\left|\Omega_{i}\right| \\
& \left.-\left(\underline{\gamma}\left(\rho^{\prime n-1 / 2}-\rho^{n-1 / 2}\right), \underline{\nabla} \partial \zeta^{\prime n}\right)\right\} \Delta t \\
& =\left(\underline{\gamma} \zeta^{\prime \eta} \cdot \underline{\nabla} \zeta^{\prime} \eta\right)-\sum_{n=1}^{\eta}\left(\underline{\gamma} \partial \zeta^{\prime n} \cdot \underline{\nabla} \zeta^{\cdot n-1 / 2}\right) \Delta t \\
& +\sum^{\eta}\left\{\left(\phi\left(\partial \rho^{, n}-\rho_{t}^{n-1 / 2}\right), \partial \zeta^{, n}\right)\right. \\
& n=1 \\
& +\sum_{i} \phi_{m i}\left(\partial \bar{\rho}_{m}{ }^{n} i^{n}-\bar{\rho}_{m, t, i}{ }^{n-1 / 2}\right) \partial \hat{\zeta}_{i}{ }^{n}\left|\Omega_{i}\right|
\end{aligned}
$$




$$
\begin{gathered}
\left.+\left(\underline{\gamma}\left(\partial \rho^{\prime n}-\partial \rho^{n}\right), \underline{\nabla} \zeta^{\prime n-1 / 2}\right)\right\} \Delta t \\
-\left(\underline{\gamma}\left(\rho^{\prime} \eta-\rho^{\eta}\right), \underline{\nabla} \zeta^{\prime \eta}\right) \\
\leq C\left\{E_{2}^{2}(p, q)+\max _{0 \leq n \leq N}\left\|\zeta^{\prime n}\right\|^{2} L^{2}(\Omega)+\sum_{n=1}^{N}\left\|\underline{\nabla} \zeta^{\prime n}\right\|^{2} L^{2}(\Omega) \Delta t\right\} \\
+\underset{n=1}{\eta} \sum_{n=1}\left(\phi \partial \zeta^{\prime n}, \partial \zeta^{\prime n}\right) \Delta t+1 / 4 B\left(\zeta^{\prime} \eta, \zeta^{\prime} \eta\right) .
\end{gathered}
$$

where we use summation by parts twice in the second equality above.

Now, by adding (6.19) and (6.20) together, making the obvious cancellations, and using the previous estimate (6.17) (with $j=0$ ), we get the other set of error estimates:

$$
\sum_{n=1}^{N}\left\|\partial \zeta^{\prime n}\right\|^{2} L^{2}(\Omega) \Delta t+\max _{0 \leq n \leq N}\left\|\underline{\nabla} \zeta^{\prime n}\right\|^{2} L^{2}(\Omega) \leq C E_{2}^{2}(p, q)
$$

and

$$
\sum_{n=1}^{N}\left\|\partial \xi^{\prime n}\right\|^{2} L^{2}\left(\Omega_{m}\right) \Delta t+\max _{0 \leq n \leq N}\left\|\nabla \xi^{\mu n}\right\|^{2} L^{2}\left(\Omega_{m}\right) \leq C E_{2}^{2}(p, q) .
$$

Finally, if we combine (6.16), (6.17), (6.21), and (6.22) with the elliptic estimates (6.6) and (6.7), we obtain the approximation error estimates:

\section{Theorem 5:}

For $\Delta t$ sufficiently small, the solution $(U, V)$ of the finite element method (5.4) through (5.7) approximates the solution $\left(\rho . \rho_{m}\right.$ ) of the double porosity model (1.4) through (1.7) as follows:

$$
\max _{0 \leq n \leq N}\left\|\rho^{n}-U^{n}\right\|_{L} 2(\Omega)
$$

$$
\begin{aligned}
& \leq C\left\{\left[\|\rho\|_{L^{\infty}(J ; H P(\Omega))}+\left\|\rho_{t}\right\|_{L^{2}\left(J ; \tilde{H}^{D-1}(\Omega)\right)}\right] h^{P}\right. \\
& \left.\quad+\left\|\rho_{m}, t\right\|_{L^{2}(J ; H}\left(\Omega_{m}\right)\right)_{m}{ }^{q^{+1}} \\
& \quad+\left[\left\|\partial^{3} \rho / \partial t^{3}\right\|_{L^{2}\left(J ; H^{-1}(\Omega)\right)}+\left\|\partial^{3} \rho_{m} / \partial t^{3}\right\|_{L^{2}\left(J ; H^{-1}\left(\Omega_{m}\right)\right)} K(\Delta t)^{2}\right\}
\end{aligned}
$$


$\max \left\|\underline{\nabla}(\rho-U)^{n}\right\|_{L^{2}(\Omega)}$

\section{$0 \leq n \leq N$}

(6.23b)

$\leq C\left\{\left[\|\|_{L} \infty\left(J ; H^{P}(\Omega)\right)+\left\|\rho_{t}\right\|_{L} 2\left(J ; \mathbb{H}^{\mathrm{P}-1}(\Omega)\right)\right]\right]^{\mathrm{P}-1}$

$\left.+\left\|\rho_{m, t}\right\|_{L 2(J ; H} \alpha\left(\Omega_{m}\right)\right)_{m}{ }^{q}$

$\left.+\left[\left\|\partial^{3} \rho / \partial t^{3}\right\|_{L^{2}\left(J ; L^{2}(\Omega)\right)}+\left\|\partial^{3} P_{m} / \partial t^{3}\right\|_{L^{2}\left(J: L^{2}\left(\Omega_{m}\right)\right)}\right](\Delta t)^{2}\right\}$

$\sqrt{\sum_{n=1}^{N}\left\|\partial(\rho-U)^{n}\right\|^{2} L^{2}(\Omega) \Delta t}$

(6.23c)

$\leq C\left\{\left[\|\rho\|_{L^{\infty}(J ; H P(\Omega))}+\left\|\rho_{t}\right\|_{L^{2}(J ; H P(\Omega))}\right]\right] P$

$\left.+\left\|\rho_{m, t}\right\|_{L 2(J: H} q\left(\Omega_{m}\right)\right)_{m}{ }^{n}{ }^{q}$

$\left.+\left[\left\|\partial^{3} \rho / \partial t^{3}\right\|_{L^{2}\left(J: L^{2}(\Omega)\right)}+\left\|\partial^{3} \rho_{m} / \partial t^{3}\right\|_{L^{2}\left(J: L^{2}\left(\Omega_{m}\right)\right)}\right](\Delta t)^{2}\right\}$

$\max \left\|\rho_{m}{ }^{n}-v^{n}\right\|_{L^{2}\left(\Omega_{m}\right)}$

$0 \leq n \leq N$

(6.24a)

$\leq C\left\{\left[\|\|_{L}^{\infty}(J: H P(\Omega))+\left\|\rho_{t}\right\|_{L} 2\left(J ; \mathbb{A P}^{-1}(\Omega)\right)\right] h P\right.$

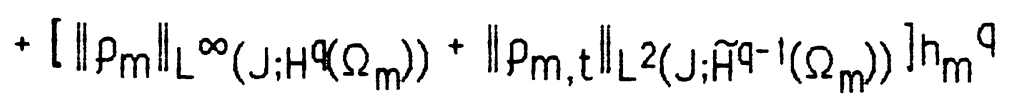

$\left.+\left[\left\|\partial^{3} \rho / \partial t^{3}\right\|_{\left.L^{2}\left(J ; H^{-1}(\Omega)\right)\right)^{+}}\left\|\partial^{3} \rho_{m} / \partial t^{3}\right\|_{L}\left(J \cdot H^{-1}\left(\Omega_{m}\right)\right)\right](\Delta t)^{2}\right\}$

$\max \left\|\nabla\left(\rho_{m}-V\right)^{n}\right\|_{L^{2}\left(\Omega_{m}\right)}$

$0 \leq n \leq N$

(6.24b)

$\leq C\left\{\left[\|\rho\|_{L} \infty(J ; H P(\Omega))+\left\|\rho_{t}\right\|_{L} 2(J ; H P(\Omega))\right] H P\right.$

$+\left[\left\|\rho_{m}\right\|_{L^{\infty}}\left(J ; H q\left(\Omega_{m}\right)\right)+\left\|\rho_{m, t}\right\|_{L 2}\left(J ; \tilde{H}^{q^{-1}}\left(\Omega_{m}\right)\right)\right] n_{m}{ }^{q-1}$

$\left.+\left[\left\|\partial^{3} \rho / \partial t^{3}\right\|_{L^{2}\left(J: L^{2}(\Omega)\right)}+\left\|\partial^{3} \rho_{m} / \partial t^{3}\right\|_{L^{2}\left(J: L^{2}\left(\Omega_{m}\right)\right)}\right](\Delta t)^{2}\right\}$

$\sqrt{\sum_{n=1}^{N}\left\|\partial\left(\rho_{m}-v\right)^{n}\right\|^{2} L^{2}\left(\Omega_{m}\right) \Delta t}$

(6.24c)

$\leq c\left\{\left[\|\|_{L}{ }_{L}^{\infty}(J ; H P(\Omega))+\left\|\rho_{t}\right\|_{L}(J ; H P(\Omega))\right] \ln P\right.$ 


$$
\begin{aligned}
& +\left\|\rho_{m, t}\right\|_{L 2\left(J ; H^{q}\left(\Omega_{m}\right)\right)} n_{m}{ }^{q} \\
& \left.+\left[\left\|\partial^{3} \rho / \partial t^{3}\right\|_{L^{2}\left(J ; L^{2}(\Omega)\right)}+\left\|\partial^{3} P_{m} / \partial t^{3}\right\|_{L^{2}\left(J ; L^{2}\left(\Omega_{m}\right)\right)}\right](\Delta t)^{2}\right\}
\end{aligned}
$$

where $1 \leq p \leq r, 1 \leq q \leq s$, and $\tilde{H} j=H^{\max }(1, j)$.

Note that the error estimates are optimal with respect to both the discretization parameters and the regularity required of the solution.

The restriction on $\Delta t$ arises from the use of Gronwall's inequality, which was, in fact, only used to bound the main gravitational term in (6.10a). The size of this restriction is independent of $h$ and $n_{m}$, and it is proportional to the reciprocal of the magnitude of the gravity coefficient $\underline{\gamma}$. Since the effect of gravity is small (assumption 2), the restriction on the size of $\Delta t$ should not be very important in practice. Further, if gravity is neglected, a careful analysis of the above proof shows that no restriction on $\Delta t$ arises at all.

\section{Acknow ledgement.}

The author would like to thank his advisor, Jim Douglas, Jr., for his encouragement and guidance, as well as specifically for his help in the formulation of the model and the finite element procedure.

\section{References.}

[1] G. I. Barenblatt, lu. P. Zheltov, and I. N. Kochina, Basic concepts in the theory of seepage of homogeneous liquids in rissured rocks [strata], Prikl. Mat. Mekh., 24 (1960), Pp. 852-864. J. Appl. Math. Mech., 24 (1960), Dp. 1286-1303.

[2] A. P. Calderón, Lebesgue spaces of differentiable functions and distributions, Proc. Sympos. Pure Math., 4 (1961), Pp. 33-49.

[3] J. Douglas, Jr. and T. Dupont, Galerkin methods for parabolic equations, SIAM J. Numer. Anal., 7 (1970), pp. 575-626.

[4] A. Friedman, Partial Differential Equations of Parabolic Type, Prentice Hall, Englewood Cliffs, N.J., 1964.

[5] H. Kazemi, Pressure transient analysis or naturally fractured reservoirs with uniform fracture distribution, Soc. Pet. Eng. J. (Dec. 1969), pp. 451462.

[6] J. L. Lions and E. Magenes, Non-homogeneous Boundary Value Problems and Applications, v. 2, Springer-Verlag, Berlin, 1972. 
[7] J. A. Nitsche, Ein kriterium für die quasi-optimatitat des Ritzchen verrahrens, Numer. Math., 11 (1968), pD. 346-348.

[8] D. W. Peaceman. Fundamentals of Numerical Reservoir Simulation, Elsevier, New York, 1977.

[9] A. E. Scheidegger, The Physics of Flow Through Porous Media, 3rd edition, Univ. of Toronto Press, Toronto and Buffalo, 1974.

[10] A. de Swaan 0., Analytic solutions for determining naturally fractured reservair properties by well testing, Soc. Pet. Eng. J. (June 1976), Pp. 117-122.

[11] J. E. Warren and P. J. Root. The Dehavior of naturally fractured reservoirs, Soc. Pet. Eng. J. (Sept. 1963), pp. 245-255.

[12] M. F. Wheeler, A priori $L_{2}$ error estimates for Galerkin approximations to parabolic partial differential equations, SIAM J. Numer. Anal., 10 (1973), PP. 723-759. 


\title{
ANALYSIS OF THE SIMULATION OF SINGLE PHASE FLOW THROUGH A NATURALLY FRACTURED RESERVOIR
}

\author{
Todd Arbogast \\ Department of Mathematics \\ University of Chicago \\ Chicago, Illinois 60637
}

\begin{abstract}
The double porosity model for single phase flow through a naturally fractured reservoir is derived as a parabolic equation coupled to a series of parabolic equations. It is shown that the coupling term can be viewed as a positive-semidefinite perturbation of the time derivative, and hence it is verified that the model is well posed. A finite element method is presented to approximate the solution, and optimal order $L^{2}$ error estimates are derived.
\end{abstract}

Key words: porous media, fractured reservoir, parabolic equation, finite element method.

\section{AMS (MOS) sub ject classif ications: $35 \mathrm{~K} 99,65 \mathrm{~N} 30,76505$.}

\section{Introduction.}

The double porosity model of single phase flow through a naturally fractured reservoir was first described by Barenblatt, Zheltov, and Kochina [1], and Warren and Root [11], each of whom assumed a quasi-steady state flow in the matrix. Kazemi [5] and de Swaan 0 . [10] considered the fully unsteady state model. We shall derive and analyze a somewhat more general form of this double porosity model.

A fractured reservoir may be idealized as a porous medium, or matrix, having some regular system of fractures. We consider a fractured reservoir $\Omega \subset \mathbb{R}^{d}, d=2$ or 3 , in which the fracture system decomposes the reservoir into a disjoint union of matrix blocks $\Omega_{i} \subset \Omega$; that is, $\bar{\Omega}=U_{i} \bar{\Omega}_{i}$ and $\Omega_{i} \cap \Omega_{j}=\varnothing$, izj. For a naturally fractured reservoir, we may assume that the diameter of each block is small compared to the dimensions of the reservoir itself.

Because the reservoir is so large, both the matrix and the fracture system have a macroscopically fine structure, each of which may be viewed as a distinct porous medium. The matrix blocks are ordinary porous media (with impermeable rock grains) for which the physics is well known (see [8] and [9]). 
The fracture system is a porous medium whose "grains" (the matrix blocks) are permeable. We consider the matrix blocks as sources (and sinks) within the fracture medium and apply the known physics to it. The macroscopic quantities porosity, (tensor) permeability, pressure, and density can be defined in the usual way with respect to the matrix as $\phi_{m}, k_{m}, P_{m}$, and $\rho_{m}$, respectively. The corresponding quantities for the fracture medium, $\phi, \mathbf{k}, p$, and $\rho$, can be defined similarly by considering the matrix blocks to be impermeable (cf. [1] and [11]).

Several physical assumptions need to be made:

1. The fluid is of constant compressibility; that is, it satis-

fies the equation of state

$$
\rho^{-1} d \rho=c d p
$$

where $c>0$ is a constant.

2. When gravity is present, the linearization approximation

$$
\rho(\underline{x}, t)^{2}=\left[\rho_{0}(\underline{x})+\left(\rho(\underline{x}, t)-\rho_{0}(\underline{x})\right)\right]^{2} \approx \rho_{0}(\underline{x})\left[2 \rho(\underline{x}, t)-\rho_{0}(\underline{x})\right]
$$

is valid, where $\rho_{0}(\underline{x})$ is some standard fluid density distribution.

3. Each matrix block interacts only with the fracture system; matrix blocks do not directly interact with each other, nor do they interact with any external source (or sink).

4. The fluid is assumed to be uniform at the surface of each matrix block.

5. The matrix porosity and permeability are constant over each block. For each i, $\phi_{m i}$ and $\boldsymbol{k}_{\mathrm{mi}}$ shall denote $\phi_{\mathrm{m}}$ and $\boldsymbol{k}_{\mathrm{m}}$ restricted to $\Omega_{j}$, respectively.

Assumptions 1 and 2 are customarily made in reservoir simulation. Assumptions 3 through 5 reflect the usual physical situation where the matrix blocks are relatively small and the matrix has a much higher storage capacity and a much lower flow capacity than the fracture system. Assumption 5 is not necessary for the analysis below. With the obvious modifications, all the results remain true if $\phi_{m}$ and $\boldsymbol{k}_{m}$ are allowed to be smooth functions of $\underline{x}$ (except, of course, the special case considered in Theorem 2). However, we shall retain this assumption to prevent the notation from becoming cumbersome.

In the fracture system, Darcy's law, conservation of mass, (1.1), and assumption 3 give us 


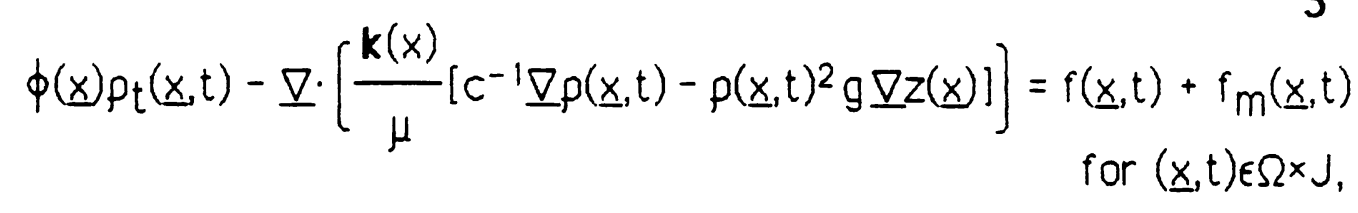

where the t subscript denotes partial differentiation in time, $\mu$ is the viscosity of the fluid, $g$ is the gravitational constant, $z$ is the vertical coordinate, $f$ and $f_{m}$ are the external and matrix source terms, respectively, and $J=(0, T]$ is the time interval of interest. By assumption 2, we linearize (1.3) as

$$
\begin{gathered}
\phi(\underline{x}) \rho_{t}(\underline{x}, t)-\underline{\nabla} \cdot\left[\frac{k(x)}{\mu c} \underline{\nabla} \rho(\underline{x}, t)-2 \rho_{0}(\underline{x}) g \frac{k(x)}{\mu} \underline{\nabla} z(\underline{x})\left[\rho(\underline{x}, t)-1 / 2 \rho_{0}(\underline{x})\right]\right] \\
=f(\underline{x}, t)+f_{m}(\underline{x}, t) \quad \text { for }(\underline{x}, t) \in \Omega x J .
\end{gathered}
$$

Since the boundary of a reservoir is generally ill-defined and of little consequence, we shall simply take the no flow Neumann condition there:

$$
\begin{aligned}
{\left[\frac{k(x)}{\mu c} \underline{\nabla} \rho(\underline{x}, t)-2 \rho_{0}(\underline{x}) g \frac{k(x)}{\mu} \underline{\nabla} z(\underline{x})\left[\rho(\underline{x}, t)-1 / 2 \rho_{0}(\underline{x})\right]\right] \cdot \underline{v}(\underline{x})=0 } \\
\\
\text { for }(\underline{x}, t) \in \partial \Omega x J,
\end{aligned}
$$

where $\underline{v}$ is the outer normal to $\partial \Omega$. Finally, the initial fluid density

$$
\rho(\underline{x}, 0)=\rho^{0}(\underline{x}) \quad \text { for } \underline{x} \in \Omega
$$

must be given.

The ith matrix block transmits through its surface an average flow of fluid

$$
f_{m i}(t)=-\frac{1}{\left|\Omega_{i}\right|} \int_{\partial \Omega_{i}} \frac{k_{m i}}{\mu c} \nabla \rho_{m} \cdot \underline{v} d s \quad \text { for } t \in J \text {, }
$$

where we use the notation $\left|\Omega_{i}\right|=\int_{\Omega_{i}} d x$ for the volume of $\Omega_{i}$. This flow should enter the fracture system near the block. In the continuous medium hypothesis, we locally average fine structure effects. This is implicitly done in defining the various macroscopic quantities above. To model the interchange between the two fine structures, we must explicitly describe the local averaging of the finer matrix structure with respect to the coarser fracture structure. Choose some partition of unity $\left\{\chi_{i}(x)\right\}$ over $\Omega$ such that each $\chi_{i}$ is approximately the characteristic function of $\Omega_{i}$ (i.e. each $X_{i}$ is supported near $\Omega_{i}, 0 \leq X_{i}, \int X_{i} d x=\left|\Omega_{i}\right|$, and 
$\left.\Sigma_{i} X_{i} \equiv 1\right)$. Now we can define the matrix source term as

$$
f_{m}(\underline{x}, t)=\sum_{i} f_{m i}(t) x_{i}(\underline{x}) \quad \text { for }(\underline{x}, t) \in \Omega \times J .
$$

The particular partition of unity used determines the exact manner in which the literal fracture flow around $\partial \Omega_{j}$ is marcoscopically spread out over the domain $\Omega$. The effect of this choice is to smooth out sharp variations in the solution $p$ that would otherwise arise near the boundary of each $\Omega_{i}$ (see Theorem 4 below). In practice, one never looks at the solution in such detail, so this choice is not actually very important.

Analogous to (1.3), we have in the i th matrix block

$$
\phi_{m i} P_{m, t}(\underline{x}, t)-\underline{\nabla} \cdot\left[\frac{k_{m}}{\mu c} \nabla P_{m}(\underline{x}, t)\right]=0 \quad \text { for }(\underline{x}, t) \in \Omega_{j} x J .
$$

Here we have omitted the gravitational term, since the effects of assumption 4 completely dominate it. Continuity of pressure requires that the block and fracture densities be equal at the surface of the block, while initial equilibrium requires the same equality at time zero. By assumption 4 , at each time $t$, we can simply take a constant, $\hat{\rho}_{j}(t)$, to represent the density of the fluid in the fractures surrounding the $i$ th block. That is, we can write

$$
P_{m}=\hat{\rho}_{i} \quad \text { on } \partial \Omega_{i} \times J
$$

(where the trace is taken from inside $\Omega_{j}$ ) and

$$
\rho_{m}(\underline{x}, 0)=\hat{\rho}^{0} i \quad \text { for } \underline{x} \in \Omega_{i} \text {. }
$$

Finally, we should define these constants according to

$$
\hat{u}_{i}=\left|\Omega_{i}\right|^{-1} \int_{\Omega} u X_{i} d x \quad \text { for } u \in L^{1}(\Omega)
$$

This definition gives us consistency between the local ( $i$ th block) behavior of the fracture flow in (1.5b) and in $(1.6 \mathrm{~b}, \mathrm{c})$.

Equations (1.4) through (1.7) constitute our model. In the next section, we shall specify the mathematical assumptions as well as define some general notation. In section 3 we analyze the matrix source term. In particular, we show that it is positive-semidef inite in some sense. In section 4 we show that 
the model is mathematically well posed. A finite element method of approx:mating the solution $\rho$ (and $\rho_{m}$ ) is presented and analyzed in sections 5 and 6 .

\section{General assumptions and notations.}

In the rest of the paper we shall use the following assumptions and notations concerning the coefficients. Assume that $f, \rho_{0}, \rho^{0}$, and the $\chi_{i}$ are smoothly distributed over the reservoir and sufficiently regular. The (unphysical) assumption on $f$ will ensure that the solution $\rho$ has the necessary smoothness and $L^{2}$-norm properties for the analysis below. Let $\phi(\underline{x})$ and $\phi_{m}$ De Dounded above and below by positive constants over $\Omega$ and $\Omega_{m}$ (where $\Omega_{m}=U_{i} \Omega_{j}$ ), respectively, and suppose that $\phi$ is smooth. Let $\boldsymbol{x}(\underline{x})=\mathbf{k}(\underline{x}) / \mu \mathrm{c}$ and $\boldsymbol{x}_{m}=\boldsymbol{k}_{m} / \mu \mathrm{c}$ be symmetric and uniformly positive-definite matrices over $\Omega$ and $\Omega_{m}$, respectively, such that $x$ is smooth and sufficiently regular. Finally, let $\underline{\gamma}(\underline{x})=$ $2 \rho_{0}(\underline{x}) g k(\underline{x}) \underline{\nabla} z(\underline{x}) / \mu$.

We will assume that $\Omega$ is a bounded domain with smooth boundary. Obviously we may not make the same assumption on $\Omega_{j}$; instead, we will assume that $\Omega_{j}$ is a convex domain, for each i. Hence, $\partial \Omega_{j}$ is piecewise smooth.

$W^{P, r}, 0 \leq p$ and $1 \leq r \leq \infty$, will denote the usual Sobolev space of $p$ times differentiable functions in $L^{r}$. Let $H^{P}=W P, 2$. $H^{-D}$ will denote the dual of $H^{P}$. $H^{Q}\left(J ; H^{P}\right), q$ a nonnegative integer, will denote the Banach space of $H^{P}$-valued functions (of $\underline{x}$ ) that are in $H^{q}$ (as functions of $t$ ). The norm of $u(\underline{x}, t) \in H^{q}\left(J ; H^{P}\right)$ is given by

$$
\|u\|_{H} Q(J ; H P)=\left\|\sum_{j=0}^{q}\right\| \partial j_{U} / \partial t\left\|_{H} P\right\|_{L}{ }^{2}(J) .
$$

Similarly we have the space $L^{\infty}(J: H P)$. We will also need the spaces $H_{0}^{1}\left(\Omega_{i}\right)=$ $\left\{v \in H^{\prime}\left(\Omega_{j}\right): v=0\right.$ on $\left.\partial \Omega_{j}\right\}$.

We will denote by $(\cdot, \cdot)$ the inner product on $L^{2}(\Omega)$ or $\left(L^{2}(\Omega)\right)^{d}$, whichever is appropriate. We will similarly denote by $(\cdot, \cdot)_{i}$ the inner product on $L^{2}\left(\Omega_{i}\right)$ or $\left(L^{2}\left(\Omega_{j}\right)\right)^{d}$, and by $(\cdot, \cdot)_{m}$ the inner product on $L^{2}\left(\Omega_{m}\right)$ or $\left(L^{2}\left(\Omega_{m}\right)\right)^{d}$. Define the symmetric bilinear forms

$$
\begin{array}{ll}
B\left(u_{1}, u_{2}\right)=\left(\boldsymbol{k} \underline{\nabla} u_{1}, \underline{\nabla} u_{2}\right) & \text { for any } u_{1}, u_{2} \in H^{\prime}(\Omega), \\
B_{1}\left(v_{1}, v_{2}\right)=\left(k_{m} \underline{\nabla} v_{1}, \underline{\nabla} v_{2}\right)_{1} & \text { for any } v_{1}, v_{2} \in H^{\prime}\left(\Omega_{1}\right), \text { for each i, }
\end{array}
$$


and

$$
B_{m}\left(v_{1}, v_{2}\right)=\left(x_{m} \underline{\nabla} v_{1}, \underline{\nabla} v_{2}\right)_{m} \quad \text { for any } v_{1}, v_{2} \in H^{\prime}\left(\Omega_{m}\right)
$$

Finally, $C$ and $\epsilon$ will denote generic positive constants.

Let us write the differential equations of the model in weak form with the above notation. First, note that by applying the divergence theorem to (1.6a), we can rewrite (1.5a) as

$$
f_{m i}(t)=-\phi_{m i} \bar{\rho}_{m, t, i}
$$

with the notation

$$
\overline{v_{i}}=\left|\Omega_{i}\right|^{-1} \int_{\Omega_{i}} v d x
$$

for the average of $v \in L^{\prime}\left(\Omega_{i}\right)$ over $\Omega_{j}$. Now we can write $(1.4 a, b)$ and (1.5) as

$$
\begin{gathered}
\left(\phi \rho_{t}, \varphi\right)+\sum_{i} \phi_{m i} \bar{\rho}_{m, t, i} \hat{\varphi}_{i}\left|\Omega_{i}\right|+B(\rho, \varphi)-(\underline{\gamma} \rho, \underline{\varphi} \varphi) \\
=(f, \varphi)-1 / 2\left(\underline{\gamma} p_{0}, \underline{\nabla} \varphi\right) \quad \text { for } \varphi \in H^{\prime}(\Omega),
\end{gathered}
$$

and, for each i, (1.6a) as

$$
\phi_{m i}\left(\rho_{m, t}, \psi\right)_{i}+B_{i}\left(\rho_{m}, \psi\right)=0 \quad \text { for } \psi \in H^{1}{ }_{0}\left(\Omega_{i}\right)
$$

of course, the boundary condition (1.6b) must be imposed on $\mathrm{Pm}_{\mathrm{m}}$.

\section{Description of the matrix source term.}

For a given $\rho$ in $H^{1}\left(J ; L^{2}(\Omega)\right.$ ), the solution $\rho_{m}$ of $(1.6)$ (or $(2.3),(1.6 \mathrm{~b}, \mathrm{c})$ ) exists and is unique as a function in $H^{1}\left(J ; L^{2}\left(\Omega_{m}\right)\right) \cap L^{2}\left(J ; H^{2}\left(\Omega_{m}\right)\right)$, since $\Omega_{j}$ is convex for each i. As a consequence, we can define the matrix source term operator $F_{m}: L^{2}\left(J ; L^{2}(\Omega)\right) \rightarrow L^{2}\left(J ; L^{2}(\Omega)\right)$ as

$$
F_{m}\left(u_{*}\right)=\sum_{i} \phi_{m i} \bar{v}_{t, i} X_{i}
$$

where, on $\Omega_{i}$, $v$ satisfies 


$$
\phi_{m i}\left(v_{t}, \psi\right)_{i}+B_{i}(v, \psi)=0 \quad \text { for } \psi \in H^{1}{ }_{0}\left(\Omega_{i}\right)
$$

$$
v=\int_{0}^{t} \hat{U}_{*}, i(\tau) d \tau \quad \text { on } \partial \Omega_{i} \times J
$$

$$
v(\underline{x}, 0)=0 \quad \text { for } \underline{x} \in \Omega_{j} .
$$

Note that $u_{*}=\rho_{t}$ implies that $v_{t}=\rho_{m}, t$; hence, $F_{m}\left(p_{t}\right)=-f_{m}$ represents flow from the fracture system to the matrix. We should expect that $\rho_{t}$ and $F_{m}\left(\rho_{t}\right)$ behave similarly, at least in some average sense. This is expressed as

Theorem 1:

$F_{m}$ is a bounded linear positive-semidefinite operator from $L^{2}\left(\mathcal{J}_{;} L^{2}(\Omega)\right)$ to $L^{2}\left(\tilde{J}: L^{2}(\Omega)\right)$ for any subinterval $\tilde{J}=(0, \tau] \subset J$. That is,

$$
\left\|F_{m}\left(U_{*}\right)\right\|_{L^{2}\left(\tilde{J} ; L^{2}(\Omega)\right)} \leq C\left\|U_{*}\right\|_{L^{2}\left(\tilde{J} ; L^{2}(\Omega)\right)}
$$

and

$$
0 \leq \int_{0}^{\tau}\left(F_{m}\left(u_{*}\right), u_{*}\right) d t
$$

for any $U_{*} \in L^{2}\left(\tilde{J} ; L^{2}(\Omega)\right)$. Moreover,

$$
-C\|u(\cdot, 0)\|^{2} L^{2}(\Omega) \leq \int_{0}^{\tau}\left(F_{m}\left(u_{t}\right), u\right) d t
$$

for any $U \in H^{1}\left(\widetilde{J} ; L^{2}(\Omega)\right)$.

Proof:

Take $\psi=v_{t}-\hat{U}_{*}, i$ in (3.2a) and integrate over $\tilde{J}$ to see

$$
\begin{aligned}
& \phi_{m i}\left\|v_{t}\right\|^{2}{ }^{2}\left(\tau_{i} L^{2}\left(\Omega_{i}\right)\right)+1 /{ }_{2} B_{i}(v, v)(\tau) \\
& \quad=\phi_{m i} \int_{0}^{\tau} \bar{v}_{t, i} \hat{u}_{*}, i d t\left|\Omega_{i}\right| \\
& \leq 1 / 2 \phi_{m i}\left\|v_{t}\right\|^{2}{ }^{2}\left(\tau_{j} L^{2}\left(\Omega_{i}\right)\right)+1 / 2 \phi_{m i}\left\|u_{*} X_{i}{ }^{1 / 2}\right\|^{2} L^{2}\left(\widetilde{J} ; L^{2}(\Omega)\right)
\end{aligned}
$$

Canceling terms above, we obtain boundedness: 
(3.7)

$$
\begin{aligned}
& \left\|F_{m}\left(U_{*}\right)\right\|_{L^{2}\left(\widetilde{J} L^{2}(\Omega)\right)} \\
& \quad=\|\| \sum_{i} \phi_{m i} \frac{v_{t}, i}{i} x_{i}\left\|_{L^{2}(\Omega)}\right\|_{L^{2}(\Im)}
\end{aligned}
$$

$$
\begin{aligned}
& \leq \sum_{i} \phi_{m i}\left\|v_{t}\right\|_{L^{2}\left(\widetilde{J} ; L^{2}\left(\Omega_{i}\right)\right)} \\
& \leq C\|U *\|_{L^{2}\left(\widetilde{J}_{i} L^{2}(\Omega)\right)} .
\end{aligned}
$$

Using only the equality in (3.6), we get positive-semidef initeness:

$$
\begin{aligned}
& \int_{0}^{\tau}\left(F_{m}\left(u_{*}\right), u_{*}\right) d t \\
& \quad=\sum_{i} \phi_{m i} \int_{0}^{\tau} \bar{v}_{t, i} \hat{u}_{*, i} d t\left|\Omega_{i}\right| \\
& \quad \geq 0 .
\end{aligned}
$$

To obtain (3.5), take $\psi=v-\int_{0} \hat{u}_{t, i} d \tau=\left(v+\hat{u}_{i}(0)\right)-\hat{u}_{i}$ in (3.2a) and integrate over $\widetilde{J}$ to see that

$$
\begin{aligned}
& 1 / 2 \phi_{m i}\left\|v+\hat{u}_{i}(0)\right\|^{2} L^{2}\left(\Omega_{i}\right)(\tau)-1 / 2 \phi_{m i}\left(\hat{u}_{i}(0)\right)^{2}\left|\Omega_{i}\right|+\int_{0}{ }^{2} B_{i}(v, v) d t \\
& \quad=\phi_{m i} \int_{0} \tau \bar{v}_{t, i} \hat{u}_{i} d t\left|\Omega_{i}\right| .
\end{aligned}
$$

and then estimate

$$
\begin{aligned}
& \int_{0}^{\tau}\left(F_{m}\left(u_{t}\right), u\right) d t \\
& \quad=\sum_{i} \phi_{m i} \int_{0}^{\tau} \bar{v}_{t, i} \hat{u}_{i} d t\left|\Omega_{i}\right| \\
& \quad \geq-C\|u(\cdot, 0)\|^{2}{ }^{2}(\Omega) .
\end{aligned}
$$

A more complete understanding of the matrix source term operator may be obtained by expressing it explicitly in terms of the auxiliary function $\theta$ which, on $\Omega_{\mathrm{i}}$, is the solution to

$$
\phi_{m i} \theta_{t}-\underline{\nabla} \cdot\left(\boldsymbol{x}_{m} \underline{\nabla} \theta\right)=0 \quad \text { on } \Omega_{\mathrm{i}} \times J \text {, }
$$

$$
\theta=1 \quad \text { on } \partial \Omega_{\mathrm{i}} \times \mathrm{J} \text {, }
$$



$(3.110)$
$\theta(\underline{x}, 0)=0 \quad$ for $\underline{x} \in \Omega_{1}$.

The solution of (1.6) is the convolution in time

$$
\rho_{m}(\underline{x}, t)=\int_{0}^{t} \theta(\underline{x}, t-\tau) \hat{\rho}_{t, i}(\tau) d \tau+\hat{\rho}_{i}^{0} \quad \text { for }(\underline{x}, t) \in \Omega_{i} \times J
$$

and so

$$
F_{m}\left(p_{t}\right)(\underline{x}, t)=\sum_{i} \phi_{m i} \int_{0}^{t} \bar{\theta}_{t, i}(t-\tau) \hat{p}_{t, i}(\tau) d \tau X_{i}(\underline{x})
$$

This, in fact, allows us to decouple the system (1.4) through (1.7). Since $\bar{\theta}_{t, 1}$ is positive, decreasing, and convex, the Fourier transform of its even extension to $\mathbb{R}$ is positive. An application of Fourier analysis to (3.13) gives an alternate proof of (3.4). This approach enables us to see that, in fact, $\left\{\int_{0}^{\top}\left(F_{m}(\cdot) \text {, }\right) d t\right\}^{1 / 2}$ is a seminorm on $L^{2}\left(J ; L^{2}(\Omega)\right)$ equivalent to the sum on $i$ of the $H^{-1 / 4}$-norms in time of the local space averages, at least in the following special (but very reasonable) case:

\section{Theorem 2:}

Suppose that each $\Omega_{i}$ is a rectangular parallelepiped whose edges are parallel to the coordinate axes, and that each $\boldsymbol{x}_{\mathrm{mi}}$ is diagonal. Then there exist positive constants $C_{1}$ and $C_{2}$ (independent of $\rho_{t}$ ) such that

$$
\begin{aligned}
& C_{1} \sum_{i}\left\|\hat{\rho}_{t, i}\right\|^{2} H^{-1 / 4}(J)\left|\Omega_{i}\right| \\
& \leq \int_{0}^{\top}\left(F_{m}\left(\rho_{t}\right), \rho_{t}\right) d t \\
& \leq C_{2} \sum_{i}\left\|\hat{\rho}_{t, i}\right\|^{2} H^{-1 / 4}(J)\left|\Omega_{i}\right| .
\end{aligned}
$$

\section{Proof:}

For each $i$, an appropriate scaling allows us to assume that $\Omega_{i}=(0, \pi)^{d}+\underline{c}_{i}$. Write

$$
\frac{1}{\phi_{m i}} x_{m i}=\left[\begin{array}{lll}
k_{1} & & 0 \\
& \ddots & \\
0 & & k_{d}
\end{array}\right] .
$$

Separation of variables applied to (3.11) gives us 


$$
\bar{\theta}_{t, i}=\left[\frac{8}{\pi^{2}}\right]_{\lambda_{1}, \ldots, \lambda_{d}}^{d} \sum_{j} \frac{\sum_{j} \lambda_{j}{ }^{2} k_{j}}{\prod_{j}^{2}} \exp \left(-\sum_{j} \lambda_{j}{ }^{2} k_{j} t\right),
$$

where $\Sigma_{0}$ denotes the sum over positive odd indices.

Let $\omega(t)=\bar{\theta}_{t, i}(|t|), t \in \mathbb{R}$, and let $\mathbf{F}$ denote the Fourier transform operator. It is trivial to calculate

$$
\mathbf{F} \omega(s)=2\left[\frac{8}{\pi^{2}}\right]^{d} \sum_{\lambda_{1}, \ldots, \lambda_{d}} \frac{\left(\sum_{j} \lambda_{j}{ }^{2} k_{j}\right)^{2}}{\left(\prod_{j} \lambda_{j}^{2}\right)\left[\left(\sum_{j} \lambda_{j}{ }^{2} k_{j}\right)^{2}+s^{2}\right]} .
$$

We will now show that there exist positive constants $\tilde{C}_{1}$ and $\tilde{C}_{2}$ such that

$$
\tilde{C}_{1}\left(1+s^{2}\right)^{-1 / 4} \leq \boldsymbol{F} \omega(s) \leq \tilde{C}_{2}\left(1+s^{2}\right)^{-1 / 4}
$$

Since $\mathbf{F} \boldsymbol{\omega}$ is continuous (Riemann-Lebesgue lemma) and even, it suffices to show the result for s large. In that case,

$$
\begin{aligned}
\mathbf{F} \omega(s) & \geq c \sum_{0} \frac{\left(\lambda_{1}{ }^{2}+d-1\right)^{2}}{\lambda_{1}{ }^{2}\left[\left(\lambda_{1}{ }^{2}+d-1\right)^{2}+s^{2}\right]} \\
& \geq \quad C \sum_{0} \frac{\lambda_{1}{ }^{2}}{\lambda_{1}{ }^{4}+s^{2}} \\
& 1 /{ }_{2} s \leq \lambda_{1}{ }^{2} \leq 3 /{ }_{2} s \\
& \geq C s^{-1 / 2},
\end{aligned}
$$

as $\lambda_{1}=O\left(s^{1 / 2}\right)$ and there are $O\left(s^{1 / 2}\right)$ terms in the last sum, and

$$
\begin{aligned}
& \left(\lambda_{1}^{2}+\sum_{j=2}^{d} \lambda_{j}^{2}\right)^{2}
\end{aligned}
$$

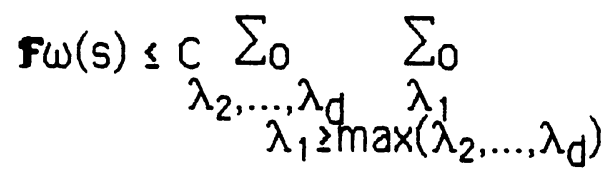

$$
\begin{aligned}
& \lambda_{1}{ }^{2}\left(\prod_{j=2}^{d} \lambda_{j}{ }^{2}\right)\left[\left(\lambda_{1}{ }^{2}+\sum_{j=2}^{d} \lambda_{j}\right)^{2}+s^{2}\right] \\
& \leq C\left[\sum_{\lambda_{2}, \ldots, \lambda_{d}} \frac{1}{\prod_{j=2}^{0} \lambda_{j}{ }^{2}}\right]\left[\sum_{\lambda_{1}} \frac{\lambda_{1}{ }^{2}}{\lambda_{1}{ }^{4}+s^{2}}\right]
\end{aligned}
$$




$$
\begin{aligned}
& \leq c \sum_{m=-\infty}^{\infty} \sum_{2_{0}} \frac{\lambda_{1}{ }^{2}}{\lambda_{s \leq \lambda_{1}{ }^{2} \leq 2^{m+i} s}} \frac{\lambda_{1}{ }^{4+s^{2}}}{2^{m}} \\
& \leq C\left[\sum_{m=-\infty}^{\infty} \frac{2^{m}}{\left(2^{2 m+1}+2^{m / 2}\right.}\right] s^{-1 / 2} \\
& \leq C s^{-1 / 2} .
\end{aligned}
$$

This establishes (3.17).

Our estimate (3.14) now follows directly from (3.13), some Fourier analy515, and (3.17):

$$
\begin{aligned}
& \int_{0}^{T}\left(F_{m}\left(\rho_{t}\right), \rho_{t}\right) d t \\
& =\sum_{i} \int_{0}^{T} \int_{0}^{t} \omega(t-\tau) \hat{\rho}_{t, i}(\tau) \hat{\rho}_{t, i}(t) d \tau d t\left|\Omega_{i}\right| \\
& =\sum \iint\left[\omega(\mathrm{t}-\tau) X_{(0, \infty)}(\mathrm{t}-\tau)\right]\left[\hat{\rho}_{\mathrm{t}, \mathrm{i}}(\tau) X_{J}(\tau)\right] \times \\
& i \quad \times\left[\hat{\rho}_{t, j}(t) \chi_{j}(t)\right] d \tau d t\left|\Omega_{i}\right| \\
& =\sum \int \boldsymbol{F}\left(\omega X_{(0, \infty)}\right)(s)\left|\boldsymbol{F}\left(\hat{p}_{t, i} X_{\jmath}\right)(s)\right|^{2} d s\left|\Omega_{i}\right| \\
& =1 / 2 \sum_{i} \int \boldsymbol{F} \omega(s)\left|\boldsymbol{F}\left(\hat{\rho}_{t, i} X_{J}\right)(s)\right|^{2} d s\left|\Omega_{i}\right|
\end{aligned}
$$

which is then equivalent to

$$
\sum_{i} \int\left(1+s^{2}\right)^{-1 / 4}\left|\boldsymbol{F}\left(\hat{\rho}_{t, i} X_{J}\right)(s)\right|^{2} d s\left|\Omega_{i}\right|
$$

Since the integral above is equivalent to the square of the $\mathrm{H}^{-1 / 4}$-norm, the proof is complete.

As a final remark in this section, we will compare the so called warren and Root model ([1] and [11]) with the present model. The quasi-steady state assumption, that $\rho_{m, t}=-\alpha\left(\rho_{m}-\rho\right)$, simply says that

$$
\rho_{m}(\underline{x}, t)=\int_{0}^{t}\left(1-e^{-\alpha(t-\tau)}\right) \rho_{t}(\underline{x}, \tau) d \tau+\rho^{0}(\underline{x}) \quad \text { for }(\underline{x}, t) \in \Omega \times J
$$

A glance at (3.12) shows that this assumption essentially amounts to a one parameter approximation of $\theta$ (or $\bar{\theta}_{j}$ ) by a single time exponential. 


\section{Demonstration that the model is well posed.}

We will now show the existence, uniqueness, and continuous dependence on the data $\left(f, \rho_{0}, \rho^{0}\right)$ of the solution $\left(\rho, \rho_{m}\right)$. This will be accomplished by applying the method of continuity to the problem (1.4) decoupled from (1.6) via (3.13). The linearization assumption (1.2) creates some difficulties when gravity is present. The equations are not strictly linear, and a given solution $u$ can arise from many different sets of data, as the reference density function $p_{0}$ is allowed to be arbitrary. To resolve these difficulties, we shall analyze the equation over a smaller data space. We must be careful to determine the dependence on $\underline{\gamma}$, since $\underline{\gamma}$ is proportional to $p_{0}$.

For each $\lambda \in[0,1]$, consider the problem

$$
\begin{aligned}
\phi(\underline{x}) u_{t}(\underline{x}, t)+\lambda F_{m}\left(u_{t}\right)(\underline{x}, t)-\underline{\nabla} \cdot[x(\underline{x}) \underline{\nabla} u(\underline{x}, t)-\underline{x}(\underline{x}) u(\underline{x}, t)] & =F(\underline{x}, t) \\
& \text { for }(\underline{x}, t) \in \Omega x J,
\end{aligned}
$$

$$
[x(\underline{x}) \underline{\nabla} u(\underline{x}, t)-\underline{\gamma}(\underline{x}) u(\underline{x}, t)] \cdot \underline{v}(\underline{x})=u_{0}(\underline{x}) \quad \text { for }(\underline{x}, t) \in \partial \Omega x J,
$$

$$
u(\underline{x}, 0)=u^{0}(\underline{x}) \quad \text { for } \underline{x} \in \Omega .
$$

When $\lambda=0$, we have a standard well posed problem (see [6], Theorem 5.3, p. 32); when $\lambda=1$, we have our problem (1.4), where $F(\underline{x}, t)=f(\underline{x}, t)+1 / 2 \underline{\nabla} \cdot\left[\rho_{0}(\underline{x}) \underline{\gamma}(\underline{x})\right]$, $u_{0}(\underline{x})=-1 / 2 \rho_{0}(\underline{x}) \underline{\gamma}(\underline{x}) \cdot \underline{v}(\underline{x})$, and $u^{0}(\underline{x})=\rho^{0}(\underline{x})$.

Let

$$
\begin{aligned}
\boldsymbol{v}=\left\{u \in H^{\prime}\left(J ; L^{2}(\Omega)\right) \cap L^{2}\left(J ; H^{2}(\Omega)\right):\right. \\
\quad(\boldsymbol{x} \underline{\nabla} u-\underline{\gamma} u) \cdot \underline{v} \text { on } \partial \Omega \times J \text { is independent of } t\}
\end{aligned}
$$

and

$$
\begin{aligned}
\boldsymbol{w}=\left\{\left(F, u_{0}, u^{0}\right) \in L^{2}\left(J ; L^{2}(\Omega)\right) \times H^{1 / 2}(\partial \Omega) \times H^{1}(\Omega):\right. \\
\\
\left.\quad\left(\boldsymbol{k} \underline{\nabla} u^{0}-\underline{\gamma} u^{0}\right) \cdot \underline{v}=u_{0} \text { on } \partial \Omega\right\} .
\end{aligned}
$$

We can define for each $\lambda$ the linear operator

$$
\Phi_{\lambda}: v \rightarrow w
$$

representing (4.1) as

$$
\Phi_{\lambda}(u)=\left(\phi u_{t}+\lambda F_{m}\left(u_{t}\right)-\underline{\nabla} \cdot(x \underline{\nabla} u-\underline{\gamma} u),(x \underline{\nabla} u-\underline{\gamma} u) \cdot \underline{v}, u(\cdot, 0)\right),
$$


where the two trace operators are known to be bounded ([6], Theorem 2.1, p. 9).

Lemma:

The $\Phi_{\lambda}$ are bounded above and below with bounds that are independent of $\lambda \in[0,1]$. These bounds depend on $\underline{\gamma}$ on! $y$ through its $H^{1}(\Omega)$-norm.

The following technical lemma will enable us to verify the $\underline{\gamma}$ dependence. It will be used with $\psi=\underline{\gamma}$ several times in the proof of the lemma.

Technical lemma:

If the dimension $d \leq 3$, then, for any $\epsilon>0$.

$$
\left(\psi \varphi_{1}, \varphi_{2}\right) \leq C\|\psi\|^{4} H^{1}(\Omega)\left\|\varphi_{1}\right\|^{2} L^{2}(\Omega)+\epsilon\left\{\left\|\varphi_{1}\right\|^{2} H^{1}(\Omega)+\left\|\varphi_{2}\right\|^{2} L^{2}(\Omega)\right\}
$$

and

$$
\|\underline{\nabla}(\psi \varphi)\|_{L^{2}(\Omega)} \leq C\|\psi\|^{2} H^{\prime}(\Omega)\|\varphi\|_{H^{\prime}(\Omega)}+\epsilon\|\varphi\|_{H^{2}(\Omega)}
$$

Proof:

By Hölder's inequality and the Sobolev imbedding theorem, we have:

$$
\begin{aligned}
\left(\Psi \varphi_{1}, \varphi_{2}\right) & \leq\|\psi\|_{L^{6}(\Omega)}\left\|\varphi_{1}\right\|_{L^{3}(\Omega)}\left\|\varphi_{2}\right\|_{L^{2}(\Omega)} \\
& \leq\|\Psi\|_{L^{6}(\Omega)}\left\|\varphi_{1}\right\|\left\|^{1 / 2} L^{2}(\Omega)\right\| \varphi_{1}\left\|^{1 / 2} L^{6}(\Omega)\right\| \varphi_{2} \|_{L^{2}(\Omega)} \\
& \leq C\|\Psi\|_{H^{\prime}(\Omega)}\left\|\varphi_{1}\right\|^{1 / 2} L^{2}(\Omega)\left\|\varphi_{1}\right\|\left\|^{1 / 2} H^{\prime}(\Omega)\right\| \varphi_{2} \|_{L^{2}(\Omega)} \\
& \leq C\|\psi\|^{4} H^{\prime}(\Omega)\left\|\varphi_{1}\right\|_{L^{2}(\Omega)}+\epsilon\left\{\left\|\varphi_{1}\right\|^{2} H^{\prime}(\Omega)+\left\|\varphi_{2}\right\|_{L^{2}(\Omega)}\right\} .
\end{aligned}
$$

Similarly,

$$
\begin{aligned}
\|\underline{\nabla}(\Psi \varphi)\|_{L^{2}(\Omega)} \leq & \|(\nabla \psi) \varphi\|_{L^{2}(\Omega)}+\|\psi(\underline{\nabla} \varphi)\|_{L^{2}(\Omega)} \\
& \leq\|\underline{\nabla} \Psi\|_{L^{2}(\Omega)}\|\varphi\|_{L^{\infty}(\Omega)} \\
& \quad+\|\Psi\|_{L^{6}(\Omega)}\|\underline{\nabla} \varphi\|^{1 / 2} L^{2}(\Omega)\|\underline{\nabla} \varphi\|^{1 / 2} L^{6}(\Omega) \\
& \leq C\|\psi\|_{H^{1}(\Omega)}\|\varphi\|^{1 / 2} H^{1}(\Omega)\|\varphi\|^{1 / 2} H^{2}(\Omega) \\
& \leq C\|\psi\|^{2} H^{1}(\Omega)\|\varphi\|_{H^{1}(\Omega)}+\epsilon\|\varphi\|_{H^{2}(\Omega)} .
\end{aligned}
$$

Proof of the lemma:

That the $\Phi_{\lambda}$ are bounded above follows directly from Theorem 1 and the boundedness of the trace operators. The second part of the technical lemma 
shows that the bound is proportional to $1+\lambda+\|\underline{\gamma}\|^{2} H^{\prime}(\Omega) \leq 2+\|\underline{\gamma}\|^{2} H^{\prime}(\Omega)$.

Multiplying (4.1a) by $u$ and integrating over $\Omega \times \tilde{J}, \tilde{J}=(0, \tau] \subset J$, yields

$$
\begin{aligned}
& 1 / 2(\phi u, u)(\tau)+\lambda \int_{0}^{\tau}\left(F_{m}\left(u_{t}\right), u\right) d t+\int_{0}^{\tau} B(u, u) d t \\
& =\int_{0}^{\tau}(F, u) d t+\int_{0}^{\tau} \int \partial \Omega u_{0} u d s d t+\int_{0}^{\tau}(\underline{\gamma} u, \underline{\nabla} u) d t
\end{aligned}
$$

$$
\begin{aligned}
& +1 / 2\left(\phi u^{0}, u^{0}\right) \\
\leq C & \|F\|^{2} L^{2}\left(J: L^{2}(\Omega)\right)+\left\|u_{0}\right\|^{2} H^{1 / 2}(\partial \Omega)+\left\|u^{0}\right\|^{2} L^{2}(\Omega) \\
& \left.+\int_{0} \tau(\phi u, u) d t\right\}+1 / 2 \iint_{0}^{\tau} B(u, u) d t,
\end{aligned}
$$

since the $H^{1 / 2}(\partial \Omega)$-norm of the trace of $u$ is bounded by the $H^{\prime}(\Omega)$-norm of $u$. The first part of the technical lemma shows that $C$ depends on the $H^{\prime}(\Omega)$-norm of $\underline{\gamma}$. By (3.5), we may simply omit the matrix source term on the far left side of (4.6) with respect to the inequality. Then, Gronwall's inequality gives us

$$
\begin{aligned}
& \|U\|^{2} L^{\infty}\left(J: L^{2}(\Omega)\right)+\|\nabla U\|^{2} L^{2}\left(J ; L^{2}(\Omega)\right) \\
& \leq C\left\{\|F\|^{2} L^{2}\left(J ; L^{2}(\Omega)\right)+\left\|u_{0}\right\|^{2} H^{1 / 2}(\partial \Omega)+\left\|u^{0}\right\|^{2} L^{2}(\Omega)\right\} .
\end{aligned}
$$

Next, multiplying (4.1a) by $u_{t}$ and integrating over $\Omega \times \widetilde{J}$ yields

$$
\begin{aligned}
& \int_{0}^{\tau}\left(\phi u_{t}, u_{t}\right) d t+\lambda \int_{0}^{\tau}\left(F_{m}\left(u_{t}\right), u_{t}\right) d t+1 / 2 B(u, u)(\tau) \\
&=\int_{0}^{\tau}\left(F, u_{t}\right) d t+\int_{0}^{\tau} \int_{\partial \Omega} u_{0} u_{t} d s d t+\int_{0}^{\tau}\left(\underline{\gamma} u, \underline{\nabla} u_{t}\right) d t \\
&+1 / 2 B\left(u^{0}, u^{0}\right) .
\end{aligned}
$$

Integrating in time straightforwardly and by parts gives

$$
\begin{aligned}
\int_{0}^{\tau} \int \partial \Omega & u_{0} u_{t} d s d t+\int_{0}^{\tau}\left(\underline{\gamma} u, \underline{\nabla} u_{t}\right) d t \\
=\int \partial \Omega & u_{0}\left[u(\underline{x}, \tau)-u^{0}\right] d s \\
& +(\underline{\gamma} u, \underline{\nabla} u)(\tau)-\left(\underline{\gamma} u^{0}, \underline{\nabla} u^{0}\right)-\int_{0}^{\tau}\left(\underline{\gamma} u_{t}, \underline{\nabla} u\right) d t \\
\leq C\{ & \left\|u_{0}\right\|^{2} H^{1 / 2}(\partial \Omega)+\left\|u^{0}\right\|^{2} H^{1}(\Omega) \\
& \left.+\|u\|^{2} L^{\infty}\left(\tilde{J}: L^{2}(\Omega)\right)+\|\underline{\nabla} u\|^{2} L^{2}\left(\tilde{J}: L^{2}(\Omega)\right)\right\} \\
& +1 /{ }_{4} B(u, u)(\tau)+1 / \int_{0}^{\tau}\left(\phi u_{t}, u_{t}\right) d t+\epsilon\|\underline{\nabla}\|^{2} L^{2}\left(\tilde{J} ; H^{1}(\Omega)\right),
\end{aligned}
$$

where $\epsilon$ is as small as we like and, again, $C$ depends on $\|\underline{\gamma}\|_{H^{\prime}(\Omega)}$ by the technical lemma. Combining (4.8) with (3.4), (4.9), and (4.7), we see 


$$
\begin{aligned}
& 1 / 2 \int_{0}^{\tau}\left(\phi u_{t}, u_{t}\right) d t+1 / 4 B(u, U)(\tau) \\
& \leq C\left\{\|F\|^{2} L^{2}\left(J: L^{2}(\Omega)\right)+\left\|u_{0}\right\|^{2} H^{1 / 2}(\partial \Omega)+\left\|U^{0}\right\|^{2} H^{1}(\Omega)\right. \\
& \\
& \quad+\epsilon\|u\|^{2} L^{2}\left(J: H^{2}(\Omega)\right) .
\end{aligned}
$$

Next, from (4.1a) itself. Theorem 1, and the technical lemma,

$$
\begin{aligned}
& \|\underline{\nabla} \cdot \mathbf{x} \nabla u\|_{L^{2}\left(J ; L^{2}(\Omega)\right)} \\
& \leq \mathrm{C}\left\{(1+\lambda)\left\|_{\mathrm{L}}\right\|_{L^{2}\left(\mathrm{~J} ; \mathrm{L}^{2}(\Omega)\right)}+\|u\|_{L^{2}\left(\mathrm{~J} ; H^{1}(\Omega)\right)}+\|F\|_{L^{2}\left(\mathrm{~J} ; \mathrm{L}^{2}(\Omega)\right)}\right\} \\
& +\epsilon\|u\|_{L}{ }^{2}\left(\mathrm{~J} ; H^{2}(\Omega)\right)
\end{aligned}
$$

So, for $\epsilon$ having been chosen small enough above, (4.11) combines with elliptic regularity, (4.10), and (4.7) to show that

$$
\begin{aligned}
& \left.\|u\|_{L^{2}\left(J: H^{2}(\Omega)\right.} \leq C l\|\underline{\nabla} \cdot \mathbf{x} \underline{\nabla}\|_{L^{2}\left(J ; L^{2}(\Omega)\right)}+\|u\|_{L^{2}\left(J ; H^{\prime}(\Omega)\right)}\right\} \\
& \leq C\left\{\|F\|^{2} L^{2}\left(J ; L^{2}(\Omega)\right)+\left\|U_{0}\right\|^{2} H^{1 / 2}(\partial \Omega)+\left\|U^{0}\right\|^{2} H^{1}(\Omega)\right\} \text {. }
\end{aligned}
$$

Finally, (4.12) can be used to complete the estimate (4.10) and finish the proof.

Since $\Phi_{\lambda}=\lambda \Phi_{1}+(1-\lambda) \Phi_{0}$, we can now use the method of continuity to deform the solution $\tilde{u}$ of $\Phi_{0}(\tilde{u})=\left(F, U_{0}, U^{0}\right)$ continuously to a solution $u$ of $\Phi_{1}(u)=\left(F, U_{0}, U^{0}\right)$. That is, from the lemma, if $\Phi_{\lambda_{0}}$ is an isomorphism, then $\Psi\left(u: \lambda, \lambda_{0}\right)=$ $\Phi_{\lambda_{0}}{ }^{-1}\left(\left(F, \cup_{0}, U^{0}\right)+\left(\Phi_{\lambda_{0}}-\Phi_{\lambda}\right)(U)\right)$ is a contraction mapping on $\boldsymbol{v}$ for $\left|\lambda-\lambda_{0}\right|$ small enough independently of $\lambda$ and $\lambda_{0}$. The fixed point of $\Psi$ is the solution to (4.1), so $\Phi_{\lambda}$ is also an isomorphism. After a finite number of steps from $\lambda_{0}=0$, we have:

Corollary:

$\Phi_{1}$ is an isomorphism.

\section{Theorem 3:}

If $f \in L^{2}\left(J ; L^{2}(\Omega)\right)$ and $\left(\rho_{0}\right)^{2}, \rho^{0} \in H^{1}(\Omega)$ are such that $\left[\boldsymbol{x} \underline{\nabla} \rho^{0}-\underline{\gamma}\left(\rho^{0}-1 / 2 \rho_{0}\right)\right] \cdot \underline{\nu}=0$ on $\partial \Omega$, then the double porosity model (1.4) through (1.7) has a unique solution $\left(\rho, \rho_{m}\right) \in \boldsymbol{V} \times \boldsymbol{v}_{m}, \boldsymbol{v}_{m}=H^{1}\left(J ; L^{2}\left(\Omega_{m}\right)\right) \cap L^{2}\left(J ; H^{2}\left(\Omega_{m}\right)\right)$, which varies continuously with the data: 


$$
\|\rho\|_{H^{\prime}\left(J ; L^{2}(\Omega)\right)}+\|\rho\|_{L^{2}\left(J ; H^{2}(\Omega)\right)}+\left\|\rho_{m}\right\|_{H^{\prime}\left(J ; L^{2}\left(\Omega_{m}\right)\right)}
$$

$$
\begin{aligned}
& \quad\left\|\rho_{m}\right\|_{L^{2}\left(J ; H^{2}\left(\Omega_{m}\right)\right)} \leq C\left\{\|f\|_{L^{2}\left(J ; L^{2}(\Omega)\right)}+\left\|\left(\rho_{0}\right)^{2}\right\|_{H^{\prime}(\Omega)}+\left\|\rho^{0}\right\|_{H^{\prime}(\Omega)}\right\},
\end{aligned}
$$

where $C$ depends on $\left\|\rho_{0}\right\|_{H^{\prime}}(\Omega)$ and, for solutions $\left(p_{j}, P_{m, j}\right)$ arising from data $\left(f_{j}, \rho_{0, j}, \rho_{j}^{0}\right), j=1$ and 2 ,

$$
\begin{aligned}
\left\|\rho_{1}-\rho_{2}\right\|_{H^{1}}\left(J ; L^{2}(\Omega)\right) & \left\|\rho_{1}-\rho_{2}\right\|_{L^{2}\left(J ; H^{2}(\Omega)\right)} \\
& +\left\|\rho_{m, 1}-\rho_{m, 2}\right\|_{H^{1}\left(J ; L^{2}\left(\Omega_{m}\right)\right)}+\left\|\rho_{m, 1}-\rho_{m, 2}\right\|_{L^{2}}\left(J ; H^{2}\left(\Omega_{m}\right)\right) \\
\leq C\{ & \left\|f_{1}-f_{2}\right\|_{L^{2}\left(J: L^{2}(\Omega)\right)}+\left\|\left(\rho_{0,1}\right)^{2}-\left(\rho_{0,2}\right)^{2}\right\|_{H^{1}}(\Omega) \\
& \left.+\left\|\rho_{0,1}-\rho_{0,2}\right\|_{H^{1}(\Omega)}+\left\|\rho^{0}-\rho_{1}{ }_{2}\right\|_{H^{1}(\Omega)}\right\},
\end{aligned}
$$

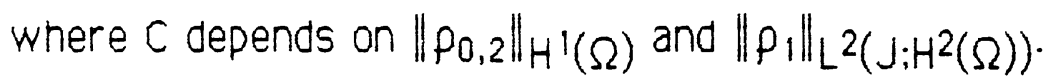

Note that Hölder's inequality and the Sobolev imbedding theorem imply that

$$
\|\Psi \varphi\|_{H^{\prime}(\Omega)} \leq C\|\Psi\|_{W^{1,12 / 5}(\Omega)}\|\varphi\|_{W^{1,12 / 5}(\Omega)} .
$$

Hence, we can replace the norm of $\left(\rho_{0}\right)^{2}$ in (4.13) by $\left\|\rho_{0}\right\|^{2} W^{11.12 / 5}(\Omega)$, and we can replace the norms involving $p_{0,1}$ and $p_{0,2}$ in $(4.14)$ by $\left(1+\left\|_{p_{0}, 1}\right\|_{W} 1,12 / 5(\Omega)\right.$ $\left.+\left\|\rho_{0,2}\right\|_{W^{1,12 / 5}(\Omega)}\right)\left\|\rho_{0,1}-\rho_{0,2}\right\|_{W^{1,12 / 5}(\Omega)}$.

Proof:

The claims for $\rho$ and $\rho_{1}-\rho_{2}$ follow directly from the remarks following (4.1) and the lemma with its corollary. Now, since $\rho$ exists and is unique, $P_{m}$ exists and is unique. If we finish the energy estimates (3.6) and (3.9) (with $u_{*}=\rho_{t}$ and $u=\rho$ so that $\left.\rho_{m}=v+\hat{\rho}_{j}\right)$, and if we use (1.6a) itself and elliptic regularity, we can bound $\rho_{m}$ in $H^{1}\left(J ; L^{2}\left(\Omega_{m}\right)\right)$ and in $L^{2}\left(J ; H^{2}\left(\Omega_{m}\right)\right)$ by

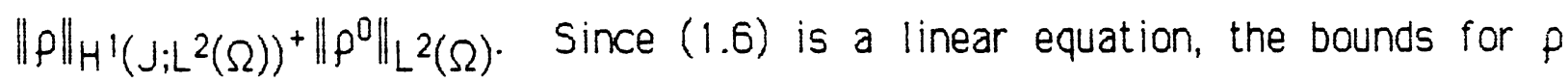
finish the proof.

Later we will want to take higher Sobolev norms of $\rho$ and $\rho_{m}$, so it is appropriate at least to say something about their smoothness here. From (1.6a) 
we see that $\rho_{m}$ is infinitely differentiable on $\Omega_{m} \times J$. Then (2.1) and (1.5b) tell us that $f_{m}$ is infinitely differentiable in time and as smooth in space as the $\chi_{\text {; }}$ are. Finally, then, (1.4a) tells us (see [4], Chapter 3, Theorem 11, p. 74)

Theorem 4:

If each of $\partial^{|\alpha|} x_{i} / \partial x^{\alpha}, \partial^{|\alpha|+}\left[\left[f+1 / 2 \underline{\nabla} \cdot\left(p_{0} \underline{\gamma}\right)\right] / \partial x^{\alpha} \partial t j, \partial^{|\alpha|} \mid \phi / \partial x^{\alpha}, \partial^{|\alpha|} x / \partial x^{\alpha}\right.$, $\partial^{|\alpha|}(\underline{\gamma}-\underline{\nabla} \cdot \mathbf{x}) / \partial x^{\alpha}$, and $\partial^{|\alpha|}(\underline{\nabla} \cdot \underline{\gamma}) / \partial x^{\alpha}$ are Hölder continuous of order $\beta \in(0,1)$ on $\Omega$ or $\Omega \times J$, as appropriate, for $0 \leq|\alpha|+2 j \leq \ell$ and $0 \leq j \leq m$ (where $\alpha$ is a multi-index), then $\partial^{|\alpha|+j} \rho / \partial x^{\alpha} \partial t j$ is Hölder continuous of order $\beta$ on $\Omega \times J$ for $0 \leq|\alpha|+2 \mid \leq l+2$ and $0 \leq j \leq m+1$.

\section{Formulation of the finite element method.}

For each $h$ and $h_{m}$ in $(0,1]$, let $\mathbf{M} \subset H^{\prime}(\Omega)$ and, for each $i, \mathbf{N}_{i} \subset H_{0}^{1}\left(\Omega_{j}\right)$ be standard Galerkin finite dimensional $H^{1}$-approximation spaces of order $r$ in $h$ and s in $n_{m}$, respectively. That is, let

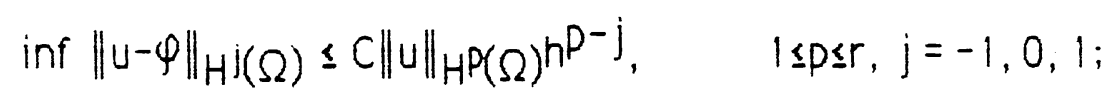

\section{$\varphi \in \mathbf{M}$}

for any $u \in H^{r}(\Omega)$ and, for each i,

$$
\text { inf }\|v-\psi\|_{H} i\left(\Omega_{j}\right) \leq C\|v\|_{H} q\left(\Omega_{j}\right)^{n_{m}}{ }^{q-j}, \quad 1 \leq q \leq s, j=-1,0,1
$$

$\Psi \in \mathbf{N}_{\mathrm{i}}$

for any $v \in H^{S}\left(\Omega_{j}\right) \cap H_{0}^{1}\left(\Omega_{j}\right)$ (where the constants $C$ are independent of $u, v, h$, and $\left.n_{m}\right)$. To avoid trivialities below, we will assume that $\boldsymbol{M}$ contains the constant functions. We will also let $\mathbf{N}_{i}^{*}=\left\{v^{*}=v+c: v \in \mathbf{N}_{i}\right.$ and $\left.c \in \mathbb{R}\right\}$. In both (5.1) and (5.2), the result for $\mathrm{j}=1$ implies the result for $\mathrm{j}=-1$ and $\mathrm{j}=0$ (see (6.6) and (6.7) below).

For $u \in H^{\prime}(\Omega)$, let $U^{\prime} \in \mathcal{M}$ denote the elliptic projection defined by

$$
B\left(u-u^{\prime}, \varphi\right)=0 \quad \text { for all } \varphi \in M
$$

and

$$
\int_{\Omega}\left(u-u^{\prime}\right) d x=0
$$


For each positive integer $\mathrm{N}$, let $\Delta t=T / N$. We will use the following notation (where $u$ is any function of time):

$$
t_{n}=n \Delta t
$$

and

$$
u^{n}=u\left(t_{n}\right)
$$

for $n=0,1, \ldots, N$, and

$$
u^{n-1 / 2}=\frac{u^{n}+u^{n-1}}{2}
$$

and

$$
\partial u^{n}=\frac{u^{n}-u^{n-1}}{\Delta t}
$$

for $n=1,2, \ldots, N$.

We shall now describe our finite element procedure. We seek a map $U:\left\{t_{0}\right.$, $\left.t_{1}, \ldots, t_{N}\right\} \rightarrow \mathcal{M}$ and, on each $\Omega_{i}$, a map $w:\left\{t_{1}, t_{2}, \ldots, t_{N}\right\} \rightarrow \mathbf{N}_{i}^{*}$ and a function $Z \in \mathbf{N}_{i}^{*}$ that satisfy the following equations:

$$
\left(\phi \partial U^{n}, \varphi\right)+\sum_{i} \phi_{m i} \partial \bar{V}_{i}^{n} \hat{\varphi}_{i}\left|\Omega_{i}\right|+B\left(u^{n-1 / 2}, \varphi\right)-\left(\underline{\gamma} u^{n-1 / 2}, \underline{\nabla} \varphi\right)
$$

$$
=\left(f^{n-1 / 2}, \varphi\right)-1 / 2\left(\underline{\gamma} \rho_{0}, \underline{\nabla} \varphi\right) \quad \text { for } \varphi \in M \text {. }
$$

$$
\phi_{m i}\left[\frac{W^{n}-v^{n-1}}{\Delta t}, \Psi\right]_{i}+B_{i}\left[\frac{W^{n}+v^{n-1}}{2}, \Psi\right]=0 \quad \text { for } \Psi \in N_{i}
$$

$$
w^{n}=\hat{U}_{i}^{n-1} \quad \text { on } \partial \Omega_{i}
$$

$$
\phi_{m i}\left[\frac{Z}{\Delta t}, \psi\right]_{i}+B_{i}\left[\frac{2}{2}, \psi\right]=0 \quad \text { for } \psi \in N_{i}
$$

$$
z=\Delta t \quad \text { on } \partial \Omega_{i} \text {, }
$$


$(5.7 a)$

$$
v^{n}=w^{n}+\partial \hat{U}_{i}^{n} z \quad \text { on } \Omega_{i}
$$

and

$$
V^{0}=\hat{u}_{i}^{0}
$$

First, we solve (5.4b) for $U^{0}$ and (5.6) for 2 . Then, successively for $n=1,2, \ldots$. $N$. we solve (5.5) for $w^{n},(5.7 a)$ for $V^{n}$ (with its explicit dependence on $U^{n}$ ), (5.4a) for $U^{n}$, and, finally, we return to (5.7a) to remove the $U^{n}$ dependence from $v^{n}$. Since uniqueness implies existence, it is not terribly hard to see that the equations can indeed be solved, provided that, if gravity is present, $\Delta t$ is not too large. This, in fact, follows from the analysis below.

It will be shown in the next section that $U$ and $V$ approximate $\rho$ and $\rho_{m}$. respectively.

Because of the enormous number of matrix blocks, the approximation procedure appears at first glance to require a massive amount of calculation. However, in actual reservoir simulation, $h$ is of necessity taken to be much larger than the diameters of the matrix blocks; consequently, the triangulation over each matrix block should be rather coarse (see Theorem 5 below), and thus the linear systems arising from (5.5) and (5.6) should be small. Since one is generally only interested in effects that are on the scale of the discretization of the fracture system, it is sufficient to solve (5.5) and (5.6) only on the blocks that sit over the quadrature points of $(5.4 a)$. If $\Omega_{j}$ is symmetric about its center, the solution $V$ is also symmetric over $\Omega_{i}$, so, with some care, one can further reduce the size of the linear systems in (5.5) and (5.6). Finally, the block problems are independent of each other by assumption 3, so naturally fractured reservoir simulation (and, in particular, our finite element procedure) is well suited to parallel type computing machines.

\section{Analysis of the approximation error.}

Let us def ine the error functions

$$
\zeta=\rho-U
$$

and

$$
\xi=\rho_{m}-V \text {. }
$$


Subtract (5.4a) from the average of (2.2) at times $t_{n}$ and $t_{n-1}$ to obtain the error equation

$$
\begin{aligned}
& \left(\phi \partial \zeta^{n}, \varphi\right)+\sum_{i} \phi_{m i} \partial \bar{\xi}_{i}^{n} \hat{\varphi}_{i}\left|\Omega_{i}\right|+B\left(\zeta^{n-i / 2}, \varphi\right)-\left(\underline{\gamma} \zeta^{n-1 / 2}, \underline{\nabla} \varphi\right) \\
& =\left(\phi\left(\partial \rho^{n}-\rho_{t}^{n-1 / 2}\right), \varphi\right)+\sum_{i} \phi_{m i}\left(\partial \bar{\rho}_{m, i} i^{n}-\bar{\rho}_{m, t, i} i^{n-i / 2}\right) \hat{\varphi}_{i}\left|\Omega_{i}\right| \\
& \quad \text { for } \varphi \in \boldsymbol{M} .
\end{aligned}
$$

$$
\zeta^{0}=\rho^{0}-\rho^{0} .
$$

Now subtract the sum of (5.5a) and $\partial \hat{u}_{i}^{n}$ times (5.6a) from the average of (2.3) at times $t_{n}$ and $t_{n-1}$ to obtain the other error equation

$$
\begin{aligned}
& \phi_{m i}\left(\partial \xi^{n}, \psi\right)_{i}+B_{i}\left(\xi^{n-1 / 2}, \psi\right) \\
& \quad=\phi_{m i}\left(\partial \rho_{m}{ }^{n}-\rho_{m, t} t^{n-1 / 2}, \psi\right)_{i} \quad \text { for } \psi \in \mathbf{N}_{i},
\end{aligned}
$$

$$
\xi^{n}=\hat{\zeta}_{i}^{n} \quad \text { on } \partial \Omega_{i} \text {, }
$$

(6.4c) $\quad \xi^{0}=\hat{\zeta}_{i}^{0}$.

Note [12] that $\zeta=\rho-\rho^{\prime}+\rho^{\prime}-U=\left(\rho-\rho^{\prime}\right)+\zeta^{\prime}$. Similarly, we can write $\xi=\left(\rho_{m}\right.$ $\left.-\rho_{m} \prime\right)+\xi "$, where we extend the following definition to all of $\Omega_{m}$; for any $v \in H^{\prime}\left(\Omega_{j}\right)$ that is constant on $\partial \Omega_{i}, v^{\prime \prime} \in \mathbf{N}_{i}{ }^{*}$ is the elliptic projection given by

$$
B_{i}\left(v-v^{\prime \prime}, \Psi\right)=0 \quad \text { for all } \Psi \in \mathbf{N}_{\mathrm{i}}
$$

and

(6.5b)

$$
v^{\prime \prime}=v \quad \text { on } \partial \Omega_{j}
$$

Elliptic theory tells us that

$$
\begin{aligned}
& \left\|\partial^{k}\left(\rho-\rho^{\prime}\right) / \partial t^{k}\right\|_{H}(\Omega) \leq C\left\|\partial^{k} \rho / \partial t^{k}\right\|_{H P(\Omega) h^{P-j},} \\
& 1 \leq P \leq r ; j=-1,0 ; k=0,1 ;
\end{aligned}
$$

$$
\left\|Z\left(\rho-\rho^{\prime}\right)\right\|_{L^{2}(\Omega)} \leq C\|\rho\|_{H P(\Omega)} h^{P^{-1}} . \quad 1 \leq p \leq r ;
$$


(6.7a)

$$
\begin{array}{r}
\left\|\partial^{k}\left(\rho_{m}-\rho_{m}\right) / \partial t^{k}\right\|_{H} j\left(\Omega_{m}\right) \leq c\left\|\partial^{k} \rho_{m} / \partial t^{k}\right\|_{H} q\left(\Omega_{m}\right)^{n}{ }_{m}^{q^{-j}}, \\
1 \leq q \leq s ; j=-1,0 ; k=0,1 ;
\end{array}
$$

and

$$
\left\|\underline{\nabla}\left(\rho_{m}-\rho_{m} "\right)\right\|_{L^{2}\left(\Omega_{m}\right)} \leq C\left\|\rho_{m}\right\|_{H} q\left(\Omega_{m}\right)^{h_{m}} q^{-1}, \quad 1 \leq q \leq s .
$$

In deriving (6.6a), the standard Nitsche duality argument [7] applies, as the smoothness of $\partial \Omega$ ensures the regularity of the dual problem. In deriving (6.7a), since $\Omega_{j}$ is convex, the dual problem is regular enough when $j=0$, but not necessarily so when $j=-1$. However, in this case, the duality argument can still be performed by posing the dual problem on a larger domain $\Omega_{i}^{*}$ with smooth boundary via the (bounded) Calderón extension operator $E: H^{1}\left(\Omega_{i}\right) \rightarrow H^{1}\left(\Omega_{i}^{*}\right)[2]$ as

$$
-\underline{\nabla} \cdot\left[\left(E x_{m}\right) \underline{\nabla} \varphi\right]=E \Psi, \quad \text { on } \Omega_{i}^{*},
$$

to obtain the needed regularity:

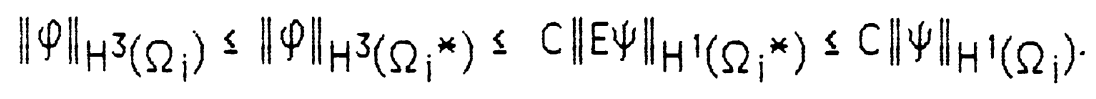

The boundary term from the integration by parts, which is normally zero by the dual problem, is now zero simply because $\rho_{m}{ }^{-} \rho_{m}{ }^{n}=0$ on $\partial \Omega_{j}$.

It remains to estimate the errors ' $\zeta^{\prime}$ and $\xi "$. Rewrite the error equations (6.3) and (6.4) in terms of these elliptic projections:

$$
\begin{gathered}
\left(\phi \partial \zeta^{\prime n}, \varphi\right)+\sum_{i} \phi_{m i} \partial \bar{\xi}_{i}^{\prime n} \hat{\varphi}_{i}\left|\Omega_{i}\right|+B\left(\zeta^{n-1 / 2}, \varphi\right)-\left(\underline{\gamma} \underline{\zeta}^{n-1 / 2}, \underline{\nabla} \varphi\right) \\
=\left(\phi\left(\partial \rho^{\prime n}-\rho_{t}^{n-1 / 2}\right), \varphi\right)+\sum_{i} \phi_{m i}\left(\partial \bar{\rho}_{m}{ }^{\prime \prime}{ }^{n}-\bar{\rho}_{m, t, i^{n-1 / 2}}\right) \hat{\varphi}_{i}\left|\Omega_{i}\right| \\
-\left(\underline{\gamma}\left(\rho^{\prime n-1 / 2}-\rho^{n-1 / 2}\right), \underline{\nabla} \varphi\right) \quad \text { for } \varphi \in \mathbb{M},
\end{gathered}
$$

(6.10D) $\quad \zeta^{\circ}=0$, 
$(6.11 a)$

$$
\phi_{m i}\left(\partial \xi^{\mu n}, \Psi\right)_{i}+B_{i}\left(\xi^{\mu n-1 / 2}, \Psi\right)
$$

$$
=\phi_{m i}\left(\partial \rho_{m}{ }^{\prime n}-\rho_{m, t}{ }^{n-1 / 2}, \psi\right)_{i} \quad \text { for } \psi \in \mathbf{N}_{i} \text {, }
$$

$(6.11 b)$

$$
\xi^{\prime \prime n}=\hat{\zeta}_{i}^{n}-\left(\hat{\rho}_{i}^{n}-\hat{\rho}_{i}^{n}\right) \quad \text { on } \partial \Omega_{i} \text {, }
$$

$$
\xi^{\prime \prime 0}=-\left(\hat{\rho}^{0}{ }_{i}-\hat{\rho}_{i}^{0}\right)
$$

It is well known how to analyze every term above ([3] and [12]) except those that represent the matrix source. We will combine these known ideas with generalizations of the ideas presented in sections 3 and 4 to accomplish our error analysis.

Let

$$
\begin{aligned}
& \left.E_{1}(P, q, j)=\left\{\|\rho\|_{L^{\infty}\left(J ; H^{+}\right.} P^{+}(\Omega)\right) h^{j-1}+\left\|\rho_{t}\right\|_{L^{2}(J ; H P(\Omega))}\right\} h^{P+1} \\
& \left.+\left\|\rho_{m, t}\right\|_{L^{2}(J ; H}{ }^{q}\left(\Omega_{m}\right)\right) h_{m}{ }^{q+1} \\
& +\left\{\left\|\partial^{3} p / \partial t^{3}\right\|_{L^{2}\left(J ; H^{-1}(\Omega)\right)}+\left\|\partial^{3} P_{m} / \partial t^{3}\right\|_{L^{2}\left(J ; H^{-1}\left(\Omega_{m}\right)\right)}\right\}(\Delta t)^{2}
\end{aligned}
$$

where, always, $1 \leq p \leq r-j, 1 \leq q \leq s$, and $j=0$ or 1 . Note that

$$
\begin{aligned}
& \text { N } \\
& \sum\left\{\left\|\partial \rho^{\prime n}-\rho_{t}{ }^{n-1 / 2}\right\|^{2} H^{-1}(\Omega)+\left\|\partial \rho_{m}{ }^{n} n-\rho_{m}, t^{n-1 / 2}\right\|^{2} H^{-1}\left(\Omega_{m}\right)\right. \\
& n=1 \\
& \left.+\sum_{i}\left[\left(\partial \hat{\rho}_{i}^{\prime}{ }^{n}-\partial \hat{\rho}_{i}^{n}\right)^{2}+\left(\partial \bar{\rho}_{m}{ }^{\prime \prime}{ }^{n}-\bar{\rho}_{m, t, i^{n-1 / 2}}\right)^{2}\right]\right\} \Delta t \\
& +\max _{0 \leq n \leq N}\left\|\rho^{\prime n}-\rho^{n}\right\|^{2} L^{2}(\Omega) \\
& \leq C E_{1}^{2}(p, q, j) \text {. }
\end{aligned}
$$

In obtaining the optimal bound on the third term on the left side above, we have used that $\chi_{i} \in H^{\prime}(\Omega)$, for all $i$.

First, take $\psi=\xi^{\prime n-1 / 2}-\left[\hat{\zeta}_{i}^{n-1 / 2}-\left(\hat{p}_{i}^{\prime}{ }^{n-1 / 2}-\hat{\rho}_{i}^{n-1 / 2}\right)\right]$ in (6.11a). After manipulating, summing on $n$ from 1 to $\eta, \eta \leq N$, and summing on $i$, we see 


$$
\begin{aligned}
& 1 /{ }_{2}\left(\phi_{m} \xi^{\prime \prime \eta}, \xi^{\prime \prime \eta}\right)_{m}-\sum_{n=1}^{\eta} \sum_{i} \phi_{m i} \partial \bar{\xi}_{i}{ }_{i}^{n} \hat{\zeta}_{i}{ }^{n-1 / 2}\left|\Omega_{i}\right| \Delta t \\
& +\sum_{n}^{\eta} B_{m}\left(\xi^{\prime \prime n-1 / 2}, \xi^{n-1 / 2}\right) \Delta t \\
& n=1 \\
& =1 / 2\left(\phi_{m} \xi^{\prime \prime}, \xi^{\prime \prime 0}\right)_{m} \\
& -\sum_{n=1}^{\eta} \sum_{i} \phi_{m i} \partial \bar{\xi}_{i}^{n}\left(\hat{\rho}_{i}^{n-1 / 2}-\hat{\rho}_{i}^{n-1 / 2}\right)\left|\Omega_{i}\right| \Delta t \\
& +\sum_{n=1}^{\eta}\left\{\left(\phi_{m}\left(\partial \rho_{m}{ }^{n}-\rho_{m}, t^{n-1 / 2}\right), \xi^{\prime n-1 / 2}\right)_{m}\right. \\
& -\sum \phi_{m i}\left(\partial \bar{\rho}_{m}{ }^{n}{ }^{n}-\bar{\rho}_{m, t, i} i^{n-1 / 2}\right)\left[\hat{\zeta}_{i}{ }^{n-1 / 2}\right. \\
& \left.\left.-\left(\hat{\rho}_{i}^{\prime}{ }^{n-1 / 2}-\hat{\rho}_{i}^{n-1 / 2}\right)\right]\left|\Omega_{i}\right|\right\} \Delta t
\end{aligned}
$$

$$
\begin{aligned}
& =-1 / 2 \sum \phi_{m i}\left(\hat{\rho}^{0}{ }_{i}-\hat{\rho}_{i}^{0}\right)^{2}\left|\Omega_{i}\right| \\
& -\sum_{i} \phi_{m i} \bar{\xi}_{i} \eta\left(\hat{\rho}_{i} \eta-\hat{\rho}_{i}^{\eta}\right)\left|\Omega_{i}\right| \\
& +\sum \sum \phi_{m i} \bar{\xi}_{i}^{n}{ }^{n-1 / 2}\left(\partial \hat{p}_{i}^{n}-\partial \hat{p}_{i}^{n}\right)\left|\Omega_{i}\right| \Delta t \\
& n=1 \text { i } \\
& +\sum_{n=1}^{\eta}\left\{\left(\phi_{m}\left(\partial \rho_{m}{ }^{\prime \prime n}-\rho_{m}, t^{n-1 / 2}\right), \xi^{\prime n-1 / 2}\right)_{m}\right. \\
& -\sum \phi_{m i}\left(\partial \bar{\rho}_{m}{ }^{n} i^{n}-\bar{\rho}_{m, t, i} i^{n-1 / 2}\right)\left[\hat{\zeta}_{i}^{n}{ }^{n-1 / 2}\right. \\
& \left.\left.-\left(\hat{p}_{i}^{\prime}{ }^{n-1 / 2}-\hat{p}_{i}^{n-1 / 2}\right)\right]\left|\Omega_{i}\right|\right\} \Delta t
\end{aligned}
$$

$$
\begin{aligned}
& \leq C E_{1}^{2}(p, q, j)+\epsilon\left\{\sum_{n=1}^{\eta}\left(\phi_{m} \xi^{\prime n-1 / 2}, \xi^{n-1 / 2}\right)_{m} \Delta t\right. \\
& \left.+\sum_{n=1}^{\eta}\left(\phi \zeta^{n-1 / 2}, \zeta^{, n-1 / 2}\right) \Delta t\right\} \\
& +1 / 4\left(\phi_{m} \xi^{\prime \prime \eta}, \xi^{\prime \prime \eta}\right)_{m}+1 / 2 \sum_{n=1}^{\eta} B_{m}\left(\xi^{\prime n-1 / 2}, \xi^{n n-1 / 2}\right) \Delta t
\end{aligned}
$$


where we have used summation by part.s in the second equality above.

Next, estimate the sum on $n$ of $(6.10 a)$ with $\varphi=\zeta^{n-1 / 2}$ to see

$(6.14)$

$$
\begin{aligned}
& 1 / 2\left(\phi \zeta^{\prime} \eta, \zeta^{\prime} \eta\right)+\sum_{n=1}^{\eta} \sum_{i} \phi_{m i} \partial \bar{\xi}_{i}^{\prime \prime} \hat{\zeta}_{i}^{\prime n-1 / 2}\left|\Omega_{i}\right| \Delta t \\
& +\sum_{n=1}^{\eta} B\left(\zeta^{n-1 / 2}, \zeta^{n-1 / 2}\right) \Delta t \\
& =\sum_{n=1}^{\eta}\left(\underline{\gamma} \zeta^{n-1 / 2}, \underline{\nabla} \zeta^{n-1 / 2}\right) \Delta t \\
& +\sum_{n=1}^{\eta}\left\{\left(\phi\left(\partial \rho^{\prime n}-\rho_{t}^{n-1 / 2}\right), \zeta^{n-1 / 2}\right)\right. \\
& n=1 \\
& +\sum_{i} \phi_{m i}\left(\partial \bar{\rho}_{m}{ }_{i}^{n}{ }^{n}-\bar{\rho}_{m, t, i}{ }^{n-1 / 2}\right) \hat{\zeta}_{i}^{n-1 / 2}\left|\Omega_{i}\right| \\
& \left.-\left(\underline{\gamma}\left(\rho^{n-1 / 2}-\rho^{n-1 / 2}\right), \underline{\nabla} \zeta^{n-1 / 2}\right)\right\} \Delta t \\
& \leq C\left\{E_{1}^{2}(p, q, j)+\sum_{n=1}^{\eta}\left(\phi \zeta^{n-1 / 2}, \zeta^{n-1 / 2}\right) \Delta t\right\} \\
& +1 / 2 \sum_{n=1}^{\eta} B\left(\zeta^{n-1 / 2}, \zeta^{\cdot n-1 / 2}\right) \Delta t
\end{aligned}
$$

We remove the coupling term between (6.13) and (6.14) by adding the two equations together. With the obvious cancellations, we obtain

$$
\begin{aligned}
& 1 / 2\left(\phi \zeta^{\prime} \eta, \zeta^{\prime \eta}\right)+1 / 2 \sum_{n=1}^{\eta} B\left(\zeta^{\prime n-1 / 2}, \zeta^{n-1 / 2}\right) \Delta t \\
& +1 / 4\left(\phi m^{\prime \prime \prime \eta}, \xi^{\prime \prime \eta}\right)_{m}+1 / 2 \sum_{n=1}^{\eta} B_{m}\left(\xi^{\prime \prime n-1 / 2}, \xi^{\prime \prime n-1 / 2}\right) \Delta t
\end{aligned}
$$

$$
\begin{aligned}
\leq C & \left\{E_{1}^{2}(p, q, j)+\sum_{n=1}^{\eta}\left(\phi \zeta^{, n-1 / 2}, \zeta^{n-1 / 2}\right) \Delta t\right\} \\
& +\in \sum_{n=1}^{\eta}\left(\phi_{m} \xi^{n n-1 / 2}, \xi^{n n-1 / 2}\right)_{m} \Delta t .
\end{aligned}
$$

If $\Delta t$ is not too large, the discrete form of Gronwall's inequality can be applied to (6.15) to yield, after some manipulation, the error estimates 


$$
\max _{0 \leq n \leq N}\left\|\xi^{n \cap}\right\|^{2} L^{2}\left(\Omega_{m}\right)+\sum_{n=1}^{N}\left\|\underline{z} \xi^{n \cap}\right\|^{2} L^{2}\left(\Omega_{m}\right) \Delta t \leq C E_{1}{ }^{2}(p, q, j)
$$

and

$$
\max _{0 \leq n \leq N}\left\|\zeta^{\prime n}\right\|^{2} L^{2}(\Omega)+\sum_{n=1}^{N}\left\|\underline{\nabla} \zeta^{\prime n}\right\|^{2} L^{2}(\Omega) \Delta t \leq C E_{1}^{2}(p, q, j) .
$$

For the next set of error estimates, let

$$
\begin{aligned}
E_{2}(P, q)= & \left\{\|\rho\|_{L^{\infty}(J ; H P(\Omega))}+\left\|p_{t}\right\|_{L^{2}(J ; H P(\Omega))}\right\} h P \\
& \left.+\left\|P_{m},\right\|_{L^{2}(J ; H} q\left(\Omega_{m}\right)\right) n_{m}{ }^{q} \\
& +\left\{\left\|\partial^{3} \rho / \partial t^{3}\right\|_{L^{2}\left(J ; L^{2}(\Omega)\right)}+\left\|\partial^{3} P_{m} / \partial t^{3}\right\|_{L^{2}\left(J ; L^{2}\left(\Omega_{m}\right)\right)}\right\}(\Delta t)^{2}
\end{aligned}
$$

where $1 \leq p \leq r$ and $1 \leq q \leq s$. Note that

$$
\begin{aligned}
& \text { N } \\
& \sum\left\{\left\|\partial \rho^{\prime n}-\rho_{t}^{n-1 / 2}\right\|^{2} L^{2}(\Omega)+\left\|\partial \rho^{\prime n}-\partial \rho^{n}\right\|^{2} L^{2}(\Omega)\right. \\
& n=1 \\
& \left.+\left\|\partial \rho_{m}{ }^{\prime n}-\rho_{m, t} t^{n-1 / 2}\right\|^{2} L^{2}\left(\Omega_{m}\right)\right\} \Delta t \\
& +\max \left\|\rho^{\prime n}-\rho^{n}\right\|^{2} L^{2}(\Omega)+E_{1}{ }^{2}(p, q, 0) \\
& 0 \leq n \leq N \\
& \leq C E_{2}^{2}(p, q) \text {. }
\end{aligned}
$$

This time, take $\psi=\partial \xi^{\prime \prime}-\left[\partial \hat{\zeta}_{i}^{\prime}{ }^{n}-\left(\partial \hat{p}^{\prime} i^{n}-\partial \hat{p}_{i}^{n}\right)\right]$ in (6.11a). Again, manipulation and summation on $n$ and $i$ yields

$$
\begin{gathered}
\sum_{n=1}^{\eta}\left(\phi_{m} \partial \xi^{\prime \prime \prime}, \partial \xi^{\prime \prime n}\right)_{m} \Delta t-\sum_{n=1}^{\eta} \sum_{i} \phi_{m i} \partial \bar{\xi}^{\prime \prime} i^{n} \partial \hat{\zeta}_{i}^{\prime n}\left|\Omega_{i}\right| \Delta t \\
+1 / 2 B_{m}(\xi " \eta, \xi " \eta)
\end{gathered}
$$


(6.19)

$$
\begin{aligned}
& =-\sum_{n=1}^{\eta} \sum_{i} \phi_{m i} \partial \bar{\xi}_{i}^{\prime \prime}{ }^{n}\left(\partial \hat{p}_{i}^{\prime}{ }^{n}-\partial \hat{p}_{i}^{n}\right)\left|\Omega_{i}\right| \Delta t \\
& \begin{array}{l}
+\sum_{n=1}^{\eta} \sum_{i}\left(\phi_{m i}\left(\partial \rho_{m}{ }^{n} n-\rho_{m, t} t^{n-1 / 2}\right),\right. \\
\left.\partial \xi^{\prime \prime n}-\left[\partial \hat{\zeta}_{i}^{n}-\left(\partial \hat{\rho^{\prime}} i^{n}-\partial \hat{\rho} i^{n}\right)\right]\right)_{i} \Delta t
\end{array} \\
& \leq C E_{2}{ }^{2}(p, q)+1 / 2 \sum_{n=1}^{\eta}\left(\phi_{m} \partial \xi^{\prime n}, \partial \xi^{\prime n}\right)_{m} \Delta t \\
& +1 / 4 \sum^{\eta}\left(\phi \partial \zeta^{n}, \partial \zeta^{\prime n}\right) \Delta t . \\
& n=1
\end{aligned}
$$

Also, take $\varphi=\partial \zeta^{, n}$ in (6.10a) and sum on $n$ to see

$$
\begin{aligned}
& \sum_{n=1}^{\eta}\left(\phi \partial \zeta^{\prime n}, \partial \zeta^{\prime n}\right) \Delta t+\sum_{n=1}^{\eta} \sum_{i} \phi_{m i} \partial \bar{\xi}^{\prime \prime} i^{n} \partial \hat{\zeta}_{i}^{\prime}{ }^{n}\left|\Omega_{i}\right| \Delta t \\
& +1 / 2 B\left(\zeta^{\prime} \eta, \zeta^{\prime} \eta\right) \\
& =\sum_{n=1}^{\eta}\left(\underline{\gamma} \zeta^{, n-1 / 2}, \underline{\nabla} \partial \zeta^{, n}\right) \Delta t \\
& +\sum_{n=1}^{\eta}\left\{\left(\phi\left(\partial \rho^{, n}-\rho_{t}^{n-1 / 2}\right), \partial \zeta^{\cdot n}\right)\right. \\
& +\sum_{i} \phi_{m i}\left(\partial \bar{\rho}_{m}{ }^{n}{ }^{n}-\bar{\rho}_{m, t, i}{ }^{n-1 / 2}\right) \partial \hat{\zeta}_{i}^{n}\left|\Omega_{i}\right| \\
& \left.-\left(\underline{\gamma}\left(\rho^{, n-1 / 2}-\rho^{n-1 / 2}\right), \underline{\nabla} \partial \zeta^{, n}\right)\right\} \Delta t \\
& =\left(\underline{\gamma} \zeta^{\prime} \eta \cdot \nabla \zeta \cdot \eta\right)-\sum_{n=1}^{\eta}\left(\underline{\gamma} \partial \zeta^{\prime n}, \nabla \zeta^{\cdot n-1 / 2}\right) \Delta t \\
& +\sum^{\eta}\left\{\left(\phi\left(\partial \rho^{\prime n}-\rho t^{n-1 / 2}\right), \partial \zeta^{\prime n}\right)\right. \\
& n=1 \\
& +\sum_{i} \phi_{m i}\left(\partial \bar{\rho}_{m}{ }_{i}^{n}-\bar{\rho}_{m, t, i}{ }^{n-1 / 2}\right) \partial \hat{\zeta}_{i}^{\prime n}\left|\Omega_{i}\right|
\end{aligned}
$$




$$
\begin{gathered}
\left.+\left(\underline{\gamma}\left(\partial \rho^{\prime n}-\partial \rho^{n}\right), \underline{\nabla} \zeta^{n-1 / 2}\right)\right\} \Delta t \\
-\left(\underline{\gamma}\left(\rho^{\prime \eta}-\rho^{\eta}\right), \underline{\nabla} \zeta^{\prime \eta}\right) \\
\leq C\left\{E_{2}^{2}(p, q)+\max _{0 \leq n \leq N}\left\|\zeta^{\prime n}\right\|{ }^{2} L^{2}(\Omega)+\sum_{n=1}^{N}\left\|\underline{\nabla} \zeta^{\prime n}\right\|{ }^{2} L^{2}(\Omega) \Delta t\right\} \\
+1 / 4 \sum_{n=1}^{\eta}\left(\phi \partial \zeta^{\prime n}, \partial \zeta^{\prime n}\right) \Delta t+1 / 4 B\left(\zeta^{\prime} \eta, \zeta^{\prime \eta}\right)
\end{gathered}
$$

where we use summation by parts twice in the second equality above.

Now, by adding (6.19) and (6.20) together, making the obvious cancellations, and using the previous estimate (6.17) (with $j=0$ ), we get the other set of error estimates:

$$
\sum_{n=1}^{N}\left\|\partial \zeta^{\prime n}\right\|^{2} L^{2}(\Omega) \Delta t+\max _{0 \leq n \leq N}\left\|\underline{\nabla} \zeta^{\prime}\right\|^{2} \|^{2}(\Omega) \leq C E_{2}^{2}(p, q)
$$

and

$$
\sum_{n=1}^{N}\|\partial \xi " n\|^{2} L^{2}\left(\Omega_{m}\right) \Delta t+\max _{0 \leq n \leq N}\left\|\nabla \xi^{\prime n}\right\|^{2} L^{2}\left(\Omega_{m}\right) \leq C E_{2}^{2}(p, q) .
$$

Finally, if we combine (6.16), (6.17), (6.21), and (6.22) with the elliptic estimates (6.6) and (6.7), we obtain the approximation error estimates:

\section{Theorem 5:}

For $\Delta t$ sufficiently small, the solution $(U, V)$ of the finite element method (5.4) through (5.7) approximates the solution $\left(\rho, \rho_{m}\right)$ of the double porosity model (1.4) through (1.7) as follows:

$$
\max _{0 \leq n \leq N}\left\|\rho^{n}-U^{n}\right\|_{L 2}(\Omega)
$$

$$
\begin{aligned}
\leq C & \left\{\left[\|\rho\|_{L}{ }^{\infty}\left(J ; H^{P}(\Omega)\right)+\left\|\rho_{t}\right\|_{L^{2}\left(J ; \tilde{H}^{-1}(\Omega)\right)}\right] h^{P}\right. \\
& \left.+\left\|\rho_{m},\right\|_{L^{2}(J ; H}{ }\left(\Omega_{m}\right)\right) n_{m}{ }^{+1} \\
& +\left[\left\|\partial^{3} \rho / \partial t^{3}\right\|_{L^{2}\left(J ; H^{-1}(\Omega)\right)}+\left\|\partial^{3} \rho_{m} / \partial t^{3}\right\|_{L^{2}\left(J ; H^{-1}\left(\Omega_{m}\right)\right)} k(\Delta t)^{2}\right\}
\end{aligned}
$$


$\max \left\|\underline{\nabla}(\rho-U)^{n}\right\|_{L^{2}(\Omega)}$

$0 \leq n \leq N$

(6.230)

$$
\begin{aligned}
\leq C & \left\{\left[\|\rho\|_{L^{\infty}}(J ; H P(\Omega))+\left\|\rho_{t}\right\|_{\left.L^{2}\left(J ; \tilde{H}^{-1}(\Omega)\right)\right] h^{P-1}}\right.\right. \\
& +\|\rho m,\|_{L} \|^{2}\left(J ; H q\left(\Omega_{m}\right)\right) n_{m}{ }^{q} \\
& \left.+\left[\left\|\partial^{3} \rho / \partial t^{3}\right\|_{L^{2}\left(J ; L^{2}(\Omega)\right)}+\left\|\partial^{3} \rho_{m} / \partial t^{3}\right\|_{L^{2}\left(J ; L^{2}\left(\Omega_{m}\right)\right)}\right](\Delta t)^{2}\right\}
\end{aligned}
$$

$\sqrt{\sum_{n=1}^{N}\left\|\partial(\rho-U)^{n}\right\|^{2} L^{2}(\Omega) \Delta t}$

(6.23c)

$$
\begin{aligned}
\leq C\{ & {\left[\|\rho\|_{L} \infty(J ; H P(\Omega))+\left\|\rho_{t}\right\|_{L^{2}(J ; H P(\Omega))}\right] h^{P} } \\
& \left.+\left\|\rho_{m, t}\right\|_{L^{2}(J ; H} q\left(\Omega_{m}\right)\right) n_{m}{ }^{q} \\
& \left.+\left[\left\|\partial^{3} \rho / \partial t^{3}\right\|_{L^{2}\left(J ; L^{2}(\Omega)\right)}+\left\|\partial^{3} \rho_{m} / \partial t^{3}\right\|_{L^{2}\left(J ; L^{2}\left(\Omega_{m}\right)\right)}\right](\Delta t)^{2}\right\}
\end{aligned}
$$

$$
\max _{0 \leq n \leq N}\left\|\rho_{m}{ }^{n}-v^{n}\right\|_{L^{2}\left(\Omega_{m}\right)}
$$

$$
\begin{aligned}
\leq C & \left\{\left[\|\rho\|_{L^{\infty}(J ; H P(\Omega))}+\left\|\rho_{t}\right\|_{\left.L^{2}\left(J ; \tilde{H}^{-1}(\Omega)\right)\right] h P}\right.\right. \\
& \left.+\left[\left\|\rho_{m}\right\|_{L^{\infty}(J ; H} q\left(\Omega_{m}\right)\right)+\left\|\rho_{m},\right\|_{L}{ }^{2}\left(J ; \tilde{H}^{q-1}\left(\Omega_{m}\right)\right)\right] h_{m}{ }^{q} \\
& \left.+\left[\left\|\partial^{3} \rho / \partial t^{3}\right\|_{L^{2}\left(J ; H^{-1}(\Omega)\right)}+\left\|\partial^{3} \rho_{m} / \partial t^{3}\right\|_{L^{2}\left(J ; H^{-1}\left(\Omega_{m}\right)\right)}\right](\Delta t)^{2}\right\}
\end{aligned}
$$

\section{$\max \left\|\underline{\nabla}\left(\rho_{m}-V\right)^{n}\right\|_{L^{2}\left(\Omega_{m}\right)}$ \\ $0 \leq n \leq N$}

$$
\begin{aligned}
\leq C & \left\{\left[\|\rho\|_{L^{\infty}}(J ; H P(\Omega))+\left\|\rho_{t}\right\|_{L^{2}(J ; H P(\Omega))}\right] h^{P}\right. \\
& +\left[\left\|\rho_{m}\right\|_{L^{\infty}(J ; H} q\left(\Omega_{m}\right)\right)+\left\|\rho_{m},\right\|_{L}{ }^{2}\left(J ; \tilde{H}^{q-1}\left(\Omega_{m}\right)\right) \\
& \left.+\left[\left\|h_{m}{ }^{3} \rho / \partial t^{3}\right\|_{L^{2}\left(J ; L^{2}(\Omega)\right)}+\left\|\partial^{3} \rho_{m} / \partial t^{3}\right\|_{L^{2}\left(J ; L^{2}\left(\Omega_{m}\right)\right)}\right](\Delta t)^{2}\right\}
\end{aligned}
$$

$$
\sqrt{\sum_{n=1}^{N}\left\|\partial\left(\rho_{m}-v\right) n\right\|^{2} L^{2}\left(\Omega_{m}\right) \Delta t}
$$

$$
\leq C\left\{\left[\|\rho\|_{L^{\infty}(J ; H P(\Omega))}+\left\|\rho_{t}\right\|_{L^{2}(J ; H P(\Omega))}\right] h^{P}\right.
$$




$$
\begin{aligned}
& +\left\|\rho_{m, t}\right\|_{L^{2}\left(J ; H^{q}\left(\Omega_{m}\right)\right) n_{m}{ }^{9}} \\
& \left.+\left[\left\|\partial^{3} \rho / \partial t^{3}\right\|_{L^{2}\left(J ; L^{2}(\Omega)\right)}+\left\|\partial^{3} P_{m} / \partial t^{3}\right\|_{L^{2}\left(J ; L^{2}\left(\Omega_{m}\right)\right)}\right](\Delta t)^{2}\right\}
\end{aligned}
$$

where $1 \leq p \leq r, 1 \leq q \leq s$, and $\tilde{H}^{j}=H^{\max }(1, j)$.

Note that the error estimates are optimal with respect to both the discretization parameters and the regularity required of the solution.

The restriction on $\Delta t$ arises from the use of Gronwall's inequality, which was, in fact, only used to bound the main gravitational term in (6.10a). The size of this restriction is independent of $h$ and $h_{m}$, and it is proportional to the reciprocal of the magnitude of the gravity coefficient $\underline{\gamma}$. Since the effect of gravity is small (assumption 2), the restriction on the size of $\Delta t$ should not be very important in practice. Further, if gravity is neglected, a careful analysis of the above proof shows that no restriction on $\Delta t$ arises at all.

\section{Acknow ledgement.}

The author would like to thank his advisor; Jim Douglas, Jr., for his encouragement and guidance, as well as specifically for his help in the formulation of the model and the finite element procedure.

\section{References.}

[1] G. I. Barenblatt, IU. P. Zheltov, and I. N. Kochina, Basic concepts in the theory or seepage of homogeneous liquids in rissured rocks [strata], Prikl. Mat. Mekh., 24 (1960), pp. 852-864. J. Appl. Math. Mech., 24 (1960), pp. 1286-1303.

[2] A. P. Calderón, Lebesgue spaces of differentiable functions and distributions, Proc. Sympos. Pure Math., 4 (1961), pp. 33-49.

[3] J. Douglas, Jr. and T. Dupont, Galerkin methods for parabolic equations, SIAM J. Numer. Anal., 7 (1970), pp. 575-626.

[4] A. Friedman, Partial Differential Equations of Parabolic Type, Prentice Hall, Englewood Cliffs, N.J., 1964.

[5] H. Kazemi, Pressure transient analysis or naturally fractured reservoirs with uniform fracture distribution, Soc. Pet. Eng. J. (Dec. 1969), Pp. 45:462.

[6] J. L. Lions and E. Magenes, Non-homogeneous Boundary Value Problems and Applications, v. 2, Springer-Verlag, Berlin, 1972. 
[7] J. A. Nitsche, Ein kriterium rür die quasi-optimatitat des fitzchen Verrahrens, Numer. Math., 11 (1968), pD. 346-348.

[8] D. W. Peaceman, Fundamentals of Numerical Reservoir Simulation, Elsevier. New York, 1977.

[9] A. E. Scheidegger, The Physics of Flow Through Porous Media, 3rd edition, Univ. of Toronto Press, Toronto and Buffalo, 1974.

[10] A. de Swaan O., Analytic solutions for determining naturally fractured reservoir properties by well testing. Soc. Pet. Eng. J. (June 1976), pp. 117-122.

[11] J. E. Warren and P. J. Root. The Dehavior or naturally rractured reservoirs, Soc. Pet. Eng. J. (Sept. 1963), pp. 245-255.

[12] M. F. Wheeler, A priori $L_{2}$ error estimates for Galerkin approximations to parabolic partial differential equations, SIAM J. Numer. Anal., 10 (1973), pp. 723-759. 